\title{
Strukturelle Untersuchung der amorph/kristallinen Grenzfläche mittels quantitativer hochauflösender Transmissionselektronenmikroskopie an den Systemen a-Si/c-Si und a-Ge/c-Si
}

\author{
Dissertation \\ zur Erlangung des Doktorgrades \\ der Mathematisch-Naturwissenschaftlichen Fakultäten \\ der Georg-August-Universität zu Göttingen
}

vorgelegt von

Karsten Thiel

aus

Bremen

Göttingen 2006 


\section{7}

Referent: Herr PD Dr. M. Seibt

Korreferent: Herr Prof. Dr. T. Salditt

Tag der mündlichen Prüfung: 02.11.2006 


\section{Inhaltsverzeichnis}

1 Einleitung 1

2 Einführung $\quad 7$

2.1 Grenzflächen zwischen geordneten und ungeordneten Materialien . . . . . 8

2.2 Methoden der QHREM . . . . . . . . . . . . . . . . . . . . 9

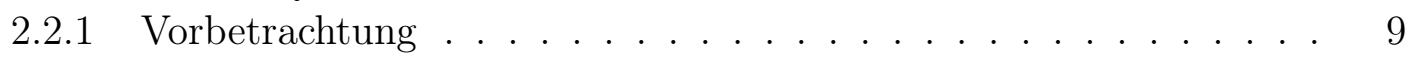

2.2.2 Kartierungsverfahren . . . . . . . . . . . . . . . . . . . . 15

2.2.3 Untersuchung der Objektwelle . . . . . . . . . . . . 15

2.3 Iterative Anpassung von Bildserien . . . . . . . . . . . . . . . 16

2.3 .1 Die Grundidee . . . . . . . . . . . . . . . . . . 17

2.3.2 Das Standardverfahren nach MöBus . . . . . . . . . . . . 18

2.3.3 Der Stobbs-Faktor . . . . . . . . . . . . . . . . . . . 19

2.3.4 Anpassung von Bildserien . . . . . . . . . . . . 20

2.4 HREM an amorph/kristallinen Grenzflächen . . . . . . . . . . . . . 21

3 Quantitative HREM an amorph/kristallinen Grenzflächen 23

3.1 Elektronenmikroskopische Kontraste an der a/c Grenzfläche . . . . . . . 24

3.2 Iterativer Bildvergleich gemittelter Grenzflächenabbildungen einer Defokusserie . . . . . . . . . . . . . . . . 26

3.2.1 Berechnung gemittelter experimenteller Grenzflächenabbildungen 26

3.2.1.1 Driftkorrektur . . . . . . . . . . . . . . 26

3.2.1.2 Mittelung von Abbildungen . . . . . . . . . . . . 27

3.2.2 Multislice-Simulation gemittelter Abbildungen . . . . . . . . . . . 30

3.2.2.1 Der Multislice-Formalismus . . . . . . . . . . . . . 30

3.2.2.2 Simulation gemittelter Grenzflächenabbildungen: Die APPNäherung . . . . . . . . . . . . . . . . . 33

3.2.2.3 Konstruktion einer zweidimensionalen Verteilungsfunktion 34

3.2.3 Vergleich simulierter und experimenteller Defokusserien . . . . . . 40 
3.2.3.1 Der Einfluss des Aufzeichnungsmediums . . . . . . . . 41

3.2.3.2 Bestimmung der Defoki und der Probendicke . . . . . . 42

3.3 Untersuchung der strukturellen Homogenität entlang der Grenzfläche . . 42

3.3.1 Die laterale Ausdehnung des Mittelungsbereiches . . . . . . . . . 43

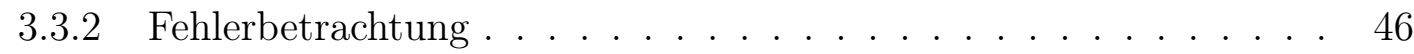

3.3.2.1 Monte-Carlo-Simulationen synthetischer Datensätze: Die

Bootstrap-Methode . . . . . . . . . . . . . 47

4 Ergebnisse $\quad 51$

4.1 Struktureller Übergang an den Grenzflächen a-Si/c-Si und a-Ge/c-Si . . . 51

4.1.1 Experimentelle gemittelte Abbildungen . . . . . . . . . . . . 52

4.1.2 Simulation gemittelter Abbildungen . . . . . . . . . . . . 55

4.1.2.1 Bestimmung der notwendigen Parameteranzahl zur Konstruktion der zweidimensionalen Verteilungsfunktion . . 58

4.1.2.2 a-Si /c-Si-Probe . . . . . . . . . . . . . 60

4.1.2.3 a-Ge/c-Si-Probe . . . . . . . . . . . . . 66

4.2 Laterale Homogenität der zweidimensionalen Verteilungsfunktion . . . . . 71

4.2 .1 a-Ge/c-Si-Probe . . . . . . . . . . . . . . . . . . . 71

4.2 .2 a-Si $/$ c-Si-Probe . . . . . . . . . . . . . . . . . . 75

5 Zusammenfassende Diskussion und Ausblick $\quad 79$

5.1 Zusammenfassende Diskussion . . . . . . . . . . . . . . . . . . . 79

5.1.1 Die Rolle der Volumenfehlpassung . . . . . . . . . . . . . . . 80

5.1.1.1 Der Einfluß auf die Standardabweichung von der mittleren Bindungsorientierung in der ersten amorphen Atomlage und die mittlere Bindungsorientierung . . . . . . . . 82

5.1.1.2 Der Einfluß auf die Weite des Übergangsbereiches . . . . 82

5.1.1.3 Die Lokalisierung der Atomverteilung . . . . . . . . . . . 83

5.1.2 Untersuchung der Homogenität der zweidimensionalen Verteilungsfunktion entlang der Grenzfläche . . . . . . . . . . . . 85

5.1.2.1 a-Ge/c-Si-Probe . . . . . . . . . . . . . . 85

$5.1 .2 .2 \quad$ a-Si/c-Si-Probe . . . . . . . . . . . . . 86

5.2 Ausblick . . . . . . . . . . . . . . . . . . . 86

\section{Anhänge}

A Vorläufige Bestimmung der Defokusschrittweite $\delta \Delta f \quad 91$

$\begin{array}{ll}\text { B Präparation der Proben } & 95\end{array}$

$\begin{array}{lr}\text { Literaturverzeichnis } & 99\end{array}$ 


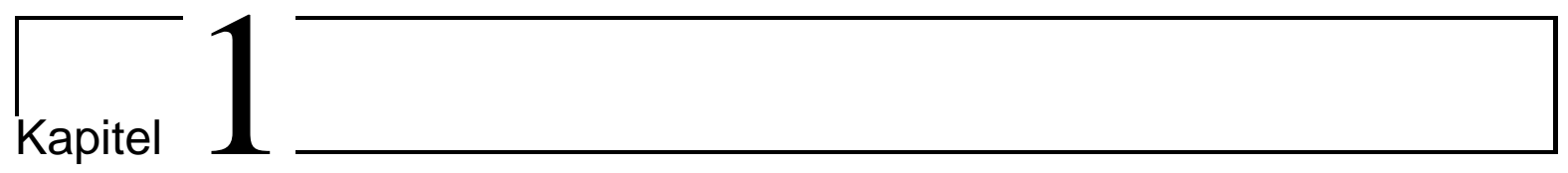

\section{Einleitung}

In der modernen Festkörperphysik stellen Heterostrukturen ein wichtiges Grundelement dar und finden in der Technologie, z.B. in Schottkydioden, Feldeffekttransistoren, Heterosolarzellen oder Speichereinheiten von Festplatten, zahlreiche Anwendung. Durch die zunehmende Miniaturisierung solcher Elemente rücken die atomare Struktur und die physikalischen Eigenschaften der Grenzflächen zwischen den Materialien immer stärker in den Fokus aktueller Forschung. Von großer Bedeutung - und dabei strukturell bisher wenig untersucht - sind Grenzflächen zwischen amorphen und kristallinen (a/c) Materialien.

Ein prominentes Beispiel ist die Grenzfläche zwischen kristallinem Silizium (Si) und amorphem Siliziumdioxid $\left(\mathrm{SiO}_{2}\right)$ in MOS-Transistoren („Metal Oxide Semiconductor“). Hier trennt eine dünne amorphe $\mathrm{SiO}_{2}$-Schicht die Gateelektrode von dem nur wenige Nanometer schmalen Leitungskanal, durch den der Strom fließt. Der Zuwachs des Leckstroms bei gleichzeitiger Verkleinerung der Oxiddicke stellt dabei eines der aktuellen Probleme in der Mikroelektronik dar. So produziert z.B. Intel Transistoren der $65 \mathrm{~nm}$ Technologie genauso wie die der 90nm Technologie mit einer Oxiddicke von $1.2 \mathrm{~nm}$, um eine Erhöhung des Leckstroms zu vermeiden ${ }^{[1]}$.

Ein weiteres Beispiel sind TMR-Elemente („Tunnel Magneto Resistance“), die sich aus dem Schichtsystem Ferromagnet/Tunnelbarriere/Ferromagnet zusammensetzen (s. z.B. Moodera et AL. ${ }^{[2]}$ ). Dabei kann entweder die Tunnelbarriere amorph und der Ferromagnet kristallin (z.B. CoFe $/ \mathrm{Al}_{2} \mathrm{O}_{3} / \mathrm{Co}$ oder $\mathrm{CoFe} / \mathrm{Al}_{2} \mathrm{O}_{3} / \mathrm{NiFe}$ ) oder die Tunnelbarriere kristallin und der Ferromagnet amorph (z.B. CoFeB $/ \mathrm{MgO} / \mathrm{CoFeB}$ ) sein. Gegenstand aktueller Forschung ist hierbei, inwieweit die atomare Struktur an den Grenzflächen den durch die Barriere transmittierten Spinstrom beeinflusst ${ }^{[3]}$. So konnten z.B. MÜNZENBERG ET AL. ${ }^{[4]}$ bereits experimentell nachweisen, dass die Verwendung ultradünner Tunnelbarrieren mit Dicken im Sub-Nanometerbereich zu einer Abnahme der Spinpolarisation führt.

Während es sich bei den beiden eben angesprochenen Beispielen um statische Grenz- 
flächen handelt, spielen dynamische Grenzflächen zwischen kristallinen und amorphen Materialien z.B. bei der Tief-Temperatur Molekularstrahlepitaxie „LT-MBE“ [5] („Low Temperature - Molecular Beam Epitaxy") oder bei Kristallisierungsprozessen von amorphen Materialien bzw. der Amorphisierung von Kristallen nach Laserbestrahlung ${ }^{[6]}$ eine Rolle.

Grundsätzlich realisiert die amorph/kristalline Grenzfläche den Übergang zwischen einem Material mit Fernordnung und einem Material mit Nahordnung bzw. mittelreichweitiger Ordnung. Aufgrund der großen Anzahl möglicher struktureller Realisierungen kann die Beschreibung der amorphen Struktur sinnvoll nur in statistischer Form erfolgen ${ }^{[7]}$. Dies geschieht für das amorphe Volumenmaterial z.B. durch die mithilfe von Beugungsmethoden bestimmbare Paarverteilungsfunktion, welche die Wahrscheinlichkeit angibt, in einem Abstand von einem bestimmten Atom ein weiteres Atom zu finden. Die Bindung des amorphen Materials an die kristallperiodisch angeordneten Atome sorgt jedoch für eine induzierte Ordnung in dem amorphen Grenzbereich, so dass durch die entstehende Anisotropie in diesem Bereich die Beschreibung der Struktur durch die Paarverteilungsfunktion nicht mehr sinnvoll ist.

Ein häufig für die Untersuchung der atomaren Struktur innerer Grenzflächen benutztes Werkzeug stellt die hochaufösende Transmissionselektronenmikroskopie „HRTEM“ oder „HREM“ („High Resolution (Transmission) Electron Microspcopy“) dar. Dabei basiert die Strukturbestimmung mittels HREM auf dem quantitativen Vergleich von Intensitätsverläufen experimenteller und simulierter Abbildungen ${ }^{[8,9]}$. Dieses Verfahren ist gut für die Untersuchung kristalliner Materialien geeignet, da für die numerische Simulation die Kenntnis der individuellen Atompositionen nötig ist. Diese Einschränkung verhindert aber die direkte Anwendung der Methode auf die Untersuchung amorpher Strukturen, die, wie bereits oben erwähnt, sinnvoll nur in Form von statistischen Verteilungsfunktionen beschrieben werden können.

Einen Ausweg bietet hier der von BorGARDT ET AL. ${ }^{[10-13]}$ vorgeschlagene Vergleich gemittelter experimenteller und simulierter Grenzflächenabbildungen. Grundlage dieser Methode ist die statistische Beschreibung der mittleren Verteilung des amorphen Materials in Grenzflächennähe durch eine dreidimensionale Verteilungsfunktion $\rho_{3 \mathrm{D}}$, welche die Wahrscheinlichkeit beschreibt, ein Atom im amorphen Material zu finden, wenn $\vec{r}=0$ die Position eines Atoms im Kristallinen ist. BorGARDT ET AL. ${ }^{[11]}$ konnten zeigen, dass entlang der Grenzfläche mit der Periode der Gitterabbildung gemittelte elektronenmikroskopische Abbildungen näherungsweise durch die zweidimensionale Projektion $\rho(x, y)$ von $\rho_{3 \mathrm{D}}$ bestimmt werden. Die gemittelten experimentellen Abbildungen können innerhalb dieser so genannten „APP“-Näherung [11] („Averaged Projected Potential“) mittels Multislice-Simulationen ${ }^{[14-18]}$ unter Verwendung von $\rho(x, y)$ für die amorphe Struktur in Grenzflächennähe direkt simuliert und mit diesen verglichen werden.

Die Beschreibung der grenzflächeninduzierten Ordnung im amorphen Material anhand einer dreidimensionalen atomaren Dichtefunktion erfolgt analog zu der Untersuchung der fest/flüssigen Grenzfläche ${ }^{[19]}$. So zeigen sowohl theoretische Untersuchungen und Simu- 


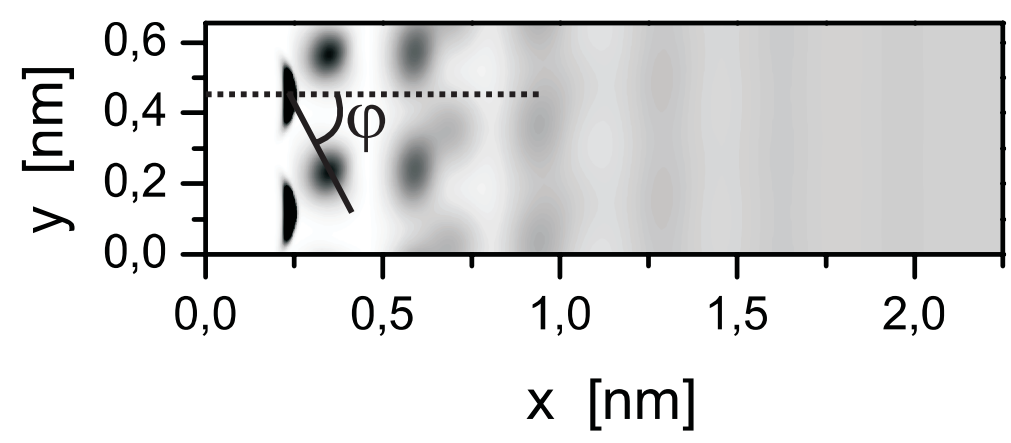

\begin{abstract}
Abbildung 1.1 - Zweidimensionale Verteilungsfunktion für die mittlere Verteilung des amorphen Germaniums an der Grenze zum kristallinen Silizium. Die Position $x=0$ steht für die letzte kristalline Atomlage. Der Winkel $\varphi$ zwischen der Grenzflächennormalen ([111]-Richtung, gepunktete Linie) und der Verbindungslinie zwischen den Schwerpunkten der ersten und zweiten Atomlage (durchgezogene Linie) beträgt für dieses Materialsystem $65^{\circ}$. Die Reichweite des Übergangsbereiches mit modifizierter amorpher Struktur wurde von BORGARDT ET AL. ${ }^{[13]}$ auf $1.4 n m$ abgeschätzt.
\end{abstract}

lationsrechnungen ${ }^{[20]}$ als auch experimentelle Beobachtungen ${ }^{[21,22]}$ eine ausgeprägte Lagenstruktur der Flüssigkeit an der Grenzfläche. Darüber hinaus konnten GEYSERMANS ET AL. ${ }^{[23]}$ anhand von Molekulardynamiksimulationen eine kristallartige Ordnung in diesen Flüssigkeitslagen vorhersagen.

Unter Verwendung dieses Ansatzes wurde von PLIKAT ${ }^{[24,25]}$ die Weite des Übergangsbereiches mit modifizierter amorpher Struktur zwischen amorphem Germanium und kristallinem Silizium auf $\approx 1 \mathrm{~nm}$ abgeschätzt. Im Rahmen seiner Dissertation wurde die amorphe Struktur näherungsweise durch eine eindimensionale mittlere Atomdichtefunktion beschrieben. BORGARDT ET AL. ${ }^{[13]}$ konnten diesen Wert durch die Verwendung der zweidimensionalen Verteilungsfunktion $\rho(x, y)$ auf $1.4 \mathrm{~nm}$ präzisieren. Abbildung 1.1 zeigt solch eine angepasste Verteilungsfunktion.

Des Weiteren wurde durch den quantitativen Vergleich gezeigt, dass der Winkel $\varphi$ zwischen der Grenzflächennormalen ([111]-Richtung) und der Verbindungslinie zwischen den Schwerpunkten der ersten und zweiten Atomlage anstelle von 70.5 , wie im Fall des ungestörten Diamantgitters, lediglich einen Wert von $65^{\circ}$ aufweist ${ }^{[13]}$. Dies wurde im Rahmen der Konstruktion der Verteilungsfunktion als tetragonale Verzerrung der mittleren Orientierung der Bindungen im Übergangsbereich interpretiert, welche in der hohen Fehlpassung zwischen dem kristallinen Silizium und dem amorphen Germanium begründet ist. Zudem ergab sich eine Standardabweichung von der mittleren Bindungsrichtung in der ersten atomaren Lage von $8.8^{\circ}[13]$, was vergleichbar mit dem Wert von $9.7^{\circ}[26]$ für die Verteilung der Bindungswinkel des reinen amorphen Germaniums ist.

Aus der Arbeit von BORGARDT ET AL. ${ }^{[13]}$ resultierten zwei offene Fragen, deren Klärung Ziel der hier vorliegenden Arbeit war. So stellte sich zum einen die Frage nach der Rolle der Volumenfehlpassung zwischen dem kristallinen Substrat und dem deponierten amorphen Material. Wie wirkt sie sich auf die amorphe Struktur in Grenzflächennähe 
aus? Wie reagiert die zweidimensionale Verteilungsfunktion darauf, und wird die Weite des Übergangsbereiches davon beeinflusst?

Im Rahmen dieser Arbeit wurde durch die Untersuchung der beiden Systeme a-Ge/c-Si $(\approx 4 \%$ Fehlpassung) und a-Si/c-Si (keine Fehlpassung) zum einen gezeigt, dass der Winkel $\varphi$ (s. o. und Abb. 1.1) im Falle des amorphen Siliziums auf kristallinem Silizium mit $70.5^{\circ}$ dem Wert des ungestörten Diamantgitters entspricht. Zum anderen ist für dieses System die Aufweitung der Verteilung der ersten atomaren Lage parallel zur Grenzfläche mit $1.9^{\circ}$ deutlich kleiner. Deswegen werden die Unterschiede in diesen Werten für beide Materialsysteme auf den Einfluss der Volumenfehlpassung zwischen dem kristallinen Silizium und dem amorphen Germanium zurückgeführt. Die Weite des Bereiches mit modifizierter amorpher Struktur ist für beide Systeme mit $\approx 1.4 \mathrm{~nm}$ vergleichbar.

Zum anderen wurde die Frage nach der lateralen Homogenität von $\rho(x, y)$ entlang der Grenzfläche untersucht. Verändert sich die Verteilungsfunktion, wenn die Grenzfläche an verschiedenen Positionen analysiert wird?

Dabei war die oben angesprochene Methode von BorGARDT ET AL. ${ }^{[10-13]}$ auf diese Fragestellung nicht direkt anwendbar, da für die statistische Beschreibung der grenzflächeninduzierten Ordnung des amorphen Materials anhand von $\rho(x, y)$ die Größe des Mittelungsbereiches per definitionem so groß wie möglich gewählt werden sollte. Die laterale Auflösung ist somit sehr klein. In der vorliegenden Arbeit wurde jedoch aus der Definition der APP-Näherung ${ }^{[1]}$ heraus eine Bedingung abgeleitet, mithilfe derer sich eine untere Grenze für die Ausdehnung des Mittelungsbereiches bestimmen ließ. Außerdem musste für den quantitativen Vergleich zweier Verteilungsfunktionen, die der Anpassung unterschiedlicher gemittelter Grenzflächenabbildungen entstammen, die Genauigkeit der angepassten Funktion $\rho(x, y)$ bestimmt werden. BORGARDT ET AL. ${ }^{[13]}$ haben auf der Basis von Testrechnungen für die Genauigkeit der unter Verwendung der APP-Methode angepassten Modellparameter bei einer Ausdehnung des Mittelungsbereiches von $9.3 \mathrm{~nm}$ zwar 5\% angegeben, jedoch wurde der Einfluss der Fehler in den Intensitäten der gemittelten Grenzflächenabbildungen auf die angepasste zweidimensionale Verteilungsfunktion dabei nicht betrachtet. Mithilfe der Bootstrap-Methode ${ }^{[27-29]}$ wurde dieser Effekt in der vorliegenden Untersuchung analysiert.

So ergab die Analyse der Homogenität der Verteilungsfunktion entlang der a-Ge/cSi-Grenzfläche, dass signifikante Variationen der atomaren Dichte nur in der zweiten und dritten Atomlage sichtbar sind. Für die erste Atomlage sind auf der Skala des Bildausschnitts keine signifikanten Unterschiede zu erkennen. Als minimale Ausdehnung des Mittelungsbereiches ergab sich $\approx 10 \mathrm{~nm}$. Aufgrund deutlicher struktureller Inhomogenitäten (kleine Inseln, Stufen) an der a-Si/c-Si-Grenzfläche konnte eine solche Analyse hier nicht durchgeführt werden. Eine sinnvolle Anpassung der Verteilungsfunktion hätte an dieser Grenzfläche nur auf einem wesentlich kleineren Mittelungsbereich erfolgen können, was aber im Widerspruch zu der statistischen Beschreibung steht.

Die vorliegende Arbeit gliedert sich somit in fünf Kapitel, wobei Kapitel 2 die Grundlagen zur Beschreibung der amorph/kristallinen Grenzfläche darstellt und zeigt, wie 
mithilfe der hochaufösenden Elektronenmikroskopie Strukturbestimmung durchgeführt werden kann. In Kapitel 3 wird das Analyseverfahren beschrieben, welchem die experimentellen elektronenmikroskopischen Abbildungen zur Klärung der oben genannten Fragen unterzogen wurden. Kapitel 4 zeigt dessen Ergebnisse. Abgeschlossen wird die Untersuchung mit Kapitel 5, in welchem alle Ergebnisse noch einmal zusammenfassend diskutiert werden und ein Ausblick auf mögliche zukünftige Untersuchungen gegeben wird. 


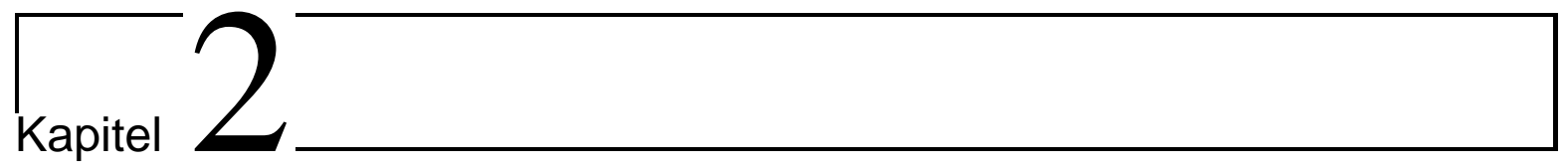

\section{Einführung}

\section{Inhaltsangabe}

2.1 Grenzflächen zwischen geordneten und ungeordneten Materialien .................. 8

2.2 Methoden der QHREM . . . . . . . . . . . . 9

2.2 .1 Vorbetrachtung . . . . . . . . . . . . . . . . . 9

2.2.2 Kartierungsverfahren . . . . . . . . . . . . . . . . . . 15

2.2.3 Untersuchung der Objektwelle . . . . . . . . . . . . . . . . 15

2.3 Iterative Anpassung von Bildserien . . . . . . . . . . 16

2.3 .1 Die Grundidee . . . . . . . . . . . . . . . . . . . . . 17

2.3.2 Das Standardverfahren nach MöBus . . . . . . . . . . . . . 18

2.3.3 Der Stobbs-Faktor . . . . . . . . . . . . . . . . . . . . . . . . . 19

2.3.4 Anpassung von Bildserien . . . . . . . . . . . . . . 20

2.4 HREM an amorph $/$ kristallinen Grenzflächen . . . . . . . . 21

In dieser Einführung werden die Grundlagen, die zum Verständnis der vorliegenden Arbeit notwendig sind, beschrieben und diskutiert. So behandelt Abschnitt 2.1 Grundsätzliches zur Beschreibung von Grenzflächen zwischen geordneten und ungeordneten Materialien, den experimentellen Zugang sowie wichtige theoretische und experimentelle Arbeiten zu den in dieser Arbeit untersuchten Grenzflächen zwischen amorphem Germanium und kristallinem Silizium sowie amorphem Silizium und kristallinem Silizium. Abschnitt 2.2 fasst kurz heute übliche Methoden zur Strukturbestimmung mittels Elektronenmikroskopie zusammen, während in Abschnitt 2.3 die in dieser Arbeit verwendete Methode erläutert wird. In Abschnitt 2.4 wird abschließend der Stand bereits durchgeführter Untersuchungen mittels Elektronenmikroskopie an amorph/kristallinen Grenzflächen diskutiert. 


\subsection{Grenzflächen zwischen geordneten und ungeordneten Materialien}

Strukturell realisiert die amorph/kristalline Grenzfläche den Übergang zwischen Materialien mit qualitativ unterschiedlicher atomarer Ordnung. Aufgrund der Translationssymmetrie zeichnet das kristalline Material eine langreichweitige Ordnung aus. Das amorphe Material hingegen besitzt eine komplexe Struktur und kann sinnvoll nur in Form von Verteilungsfunktionen und Symmetrien der lokalen Struktureinheiten beschrieben werden $^{[7]}$. Insbesondere eine Fernordnung ist hier nicht vorhanden. Stattdessen existiert eine nah- bzw. mittelreichweitige Ordnung.

Die Nahordnung beschreibt Abstände im Bereich nächster Nachbarn, also typischerweise im Bereich $<0.5 \mathrm{~nm}$. Sie kann mithilfe von Röntgen-, Neutronen- oder Elektronenbeugungsmethoden gut ermittelt werden. Dabei wird die radiale Verteilungsfunktion berechnet, die der azimuthal gemittelten Paarkorrelationsfunktion entspricht. Sie beschreibt die Wahrscheinlichkeit, in einem Abstand zu einem bestimmten Atom ein weiteres Atom im Material zu finden. Diese Funktion zeigt typischerweise ein scharfes Maximum beim Abstand des nächsten Nachbarn, verbreitert sich jedoch danach oberhalb von $\approx 1 \mathrm{~nm}$ rasch*.

Der Bereich von Abständen zwischen $0.5 \mathrm{~nm}$ und $1.5 \mathrm{~nm}$ wird als mittelreichweitige Ordnung bezeichnet. Diese kann z.B. durch die Untersuchung der Feinstruktur von Röntgenabsorptionsspektren „EXAFS“[34] („Extended X-ray-Absorption Fine Structure“) bestimmt oder durch die Fluktuationsmikroskopie ${ }^{[35-37]}$ nachgewiesen werden.

Im Übergangsbereich zwischen ferngeordnetem und ungeordnetem Material ist aufgrund der Bindung an die kristallperiodisch angeordneten Atome der Grenzfläche von langreichweitigen Korrelationen in den Atompositionen des amorphen Materials auszugehen. Diese Korrelationen klingen mit zunehmender Entfernung von der Grenzfläche ab. Eine Beschreibung durch die radiale Verteilungsfunktion ist aufgrund der Anisotropie in diesem Bereich nicht sinnvoll.

Im Gegensatz zu dem ebenfalls in dieser Arbeit untersuchten Materialsystem a-Ge/c$\mathrm{Si}$, für das keine theoretischen Vorhersagen vorliegen, existieren für das System a-Si/cSi einige theoretische Arbeiten, die zu sehr unterschiedlichen strukturellen Ergebnissen kommen. So fanden TU ET AL. ${ }^{[38]}$ mittels Monte-Carlo-Methoden für die Weite des Übergangsbereiches durch Berechnung der lokalen Energiedichte des Systems a$\mathrm{Si} / \mathrm{Si}(111)$ einen Wert von 0.3nm, der einer Si(111)-Doppellage entspricht. Die Beschreibung des amorphen Siliziums erfolgte hierbei auf der Basis eines zusammenhängenden Netzwerks $^{[39]}$ (,,continuous-random-network ${ }^{“}$ ). In einer solchen Beschreibung wird die Zufälligkeit der Atompositionen durch die Erhaltung der Koordination eingeschränkt,

\footnotetext{
*Es wurde kürzlich gezeigt, dass dieser Bereich durch die Verwendung von hochauflösenden Spektrometern in Verbindung mit kohärenter Synchrotronstrahlung sehr hoher Helligkeit erweitert werden $\operatorname{kann}{ }^{[30-33]}$.
} 
d.h. jedes Atom hat weiterhin eine feste Anzahl von Bindungen an andere Atome. Nicht abgesättigte Bindungen, so genannte „dangling bonds“, sind nicht erlaubt. Mithilfe von Tight-Binding-Simulationen berechneten BERNSTEIN ET AL. ${ }^{[40]}$ hingegen 0.7nm für das a-Si/Si(001) System. Dabei wurde die rms-Abweichung der Bindungswinkel vom idealen tetraedrischen Winkel als Maß für die Ordnung verwendet. Die Einschränkung durch die Erhaltung der Koordination war bei dieser und den folgenden Untersuchungen nicht mehr vorhanden. DA SILVA ET AL. ${ }^{[41]}$ wiederum erhielten aus Monte-Carlo-Simulationen einen Wert von 1.1nm für die Weite der Übergangsbereiches im System a-Si/Si(001). Dabei wurde ebenfalls die mittlere Abweichung vom theoretischen Bindungswinkel für die Bestimmung der Ausdehnung des Übergangsbereiches benutzt. IZUMI ET AL. ${ }^{[42]}$ schließlich haben mittels Molekulardynamik-Simulationen einen Wert von 0.8-0.9nm gefunden.

\subsection{Methoden der quantitativen hochauflösenden Transmissionselektronenmikroskopie (QHREM)}

Die Untersuchung und quantitative Charakterisierung von Strukturen mit atomarer Auflösung kann mittels der hochaufösenden Elektronenmikroskopie auf vielfältige Weise erfolgen. Abhängig von der zu untersuchenden physikalischen Eigenschaft existieren eine Vielzahl unterschiedlicher Analyseverfahren. Dabei beschränken sich diese Techniken nicht auf perfekte Kristalle. Grenzflächen zwischen verschiedenen Materialien sind, ebenso wie Defekte, mithilfe der HREM gut zu untersuchen. Die Standardverfahren können dabei grob in drei Gruppen eingeteilt werden, von denen das Kartierungsverfahren und die Untersuchung der Objektwelle in den Abschnitten 2.2.2 und 2.2.3 kurz beschrieben werden. Die für diese Arbeit verwendete Methode der iterativen Bildanpassung wird in Abschnitt 2.3 genauer betrachtet. Eine allgemeine Übersicht über die Möglichkeiten, wie quantitative Informationen durch hochauflösende Elektronenmikroskopie gewonnen werden können, bietet der Artikel von KRET ET AL. ${ }^{[43]}$.

Doch zunächst soll im folgenden Abschnitt 2.2.1 diskutiert werden, was mittels hochauflösender Elektronenmikroskopie überhaupt messbar ist, warum die Strukturbestimmung in der Regel nicht direkt anhand der Intensitätsverteilung innerhalb einer einzelnen elektronenmikroskopischen Abbildung erfolgen kann und worin die Probleme bei der Interpretation solcher Aufnahmen begründet sind.

\subsubsection{Vorbetrachtung}

Abbildung 2.1 zeigt ganz links schematisch die Entstehung elektronenmikroskopischer Abbildungen:

- Die einfallende - in guter Näherung ebene - Elektronenwelle, $\psi_{\text {in }}$, wird durch das Streupotential $V$ der Probe modifiziert, so dass an der Unterseite der Probe 


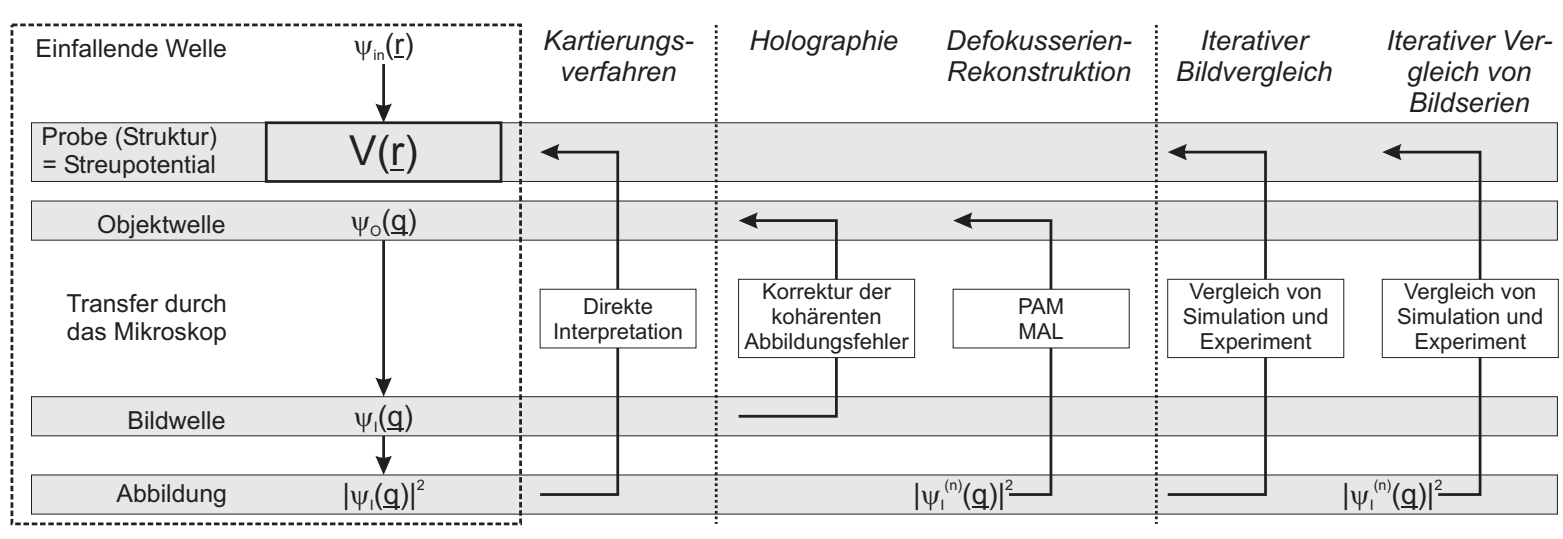

Abbildung 2.1 - Übersicht der Methoden zur Strukturbestimmung mittels HREM. Dabei steht $\psi_{\text {in }}(\vec{r})$ für die einfallende Elektronenwelle, $V(\vec{r})$ für das Streupotential der Probe, $\psi_{O}(\vec{q})$ für die die Probe verlassende komplexe Objektwelle, $\psi_{I}(\vec{q})$ für die komplexe Bildwelle, $\left|\psi_{I}(\vec{q})\right|^{2}$ für die aufgezeichnete reelle Abbildung, und $\left|\psi_{I}^{(n)}(\vec{q})\right|^{2}$ bezeichnet $n$ aufgezeichnete Abbildungen. Weitere Erläuterungen siehe Text.

- die Objektwelle, $\psi_{O}$, entsteht, die wiederum durch den Abbildungsvorgang im Elektronenmikroskop in

- die Bildwelle, $\psi_{I}$, überführt wird.

Eine konventionelle HREM-Abbildung zeichnet die Intensität in der Bildebene $I=$ $\left|\psi_{I}\right|^{2}$ auf, während bei der Elektronenholographie die Intensität der Bildwelle holographisch, d.h. überlagert mit einer Referenzwelle, gemessen wird. Ein solches Hologramm erlaubt die direkte Bestimmung der komplexwertigen Bildwelle.

Für eine Strukturbestimmung ist es notwendig, das Streupotential aus der Bildwelle (Holographie) oder der Bildintensität zu rekonstruieren, eine Aufgabe, die zu den inversen Problemen zählt ${ }^{[44]}$.

Es gibt unterschiedliche Ansätze, das Streupotential oder zumindest wichtige Eigenschaften des Streupotentials, wie z.B. die Lage seiner Maxima, zu bestimmen:

- Bei dem Kartierungsverfahren versucht man, durch die direkte Interpretation von elektronenmikroskopischen Abbildungen strukturelle oder chemische Veränderungen in der Probe zu ermitteln (s. auch Abschnitt 2.2.2).

- Bei der zweiten Vorgehensweise wird versucht, durch die „Messung“ der durch das Streupotential modulierten Elektronenwelle und die Kenntnis der einfallenden ebenen Welle das Streupotential zu rekonstruieren - wiederum ein inverses Problem (vgl. Abschnitt 2.2.3). Dabei werden zwei Methoden unterschieden:

1. Bei der Holographie-Methode wird die Objektwelle durch eine holographische Aufzeichnung der Bildwelle und eine anschließende Korrektur der kohärenten Abbildungsfehler direkt ,gemessen“. 
2. Die Defokusserien-Rekonstruktion versucht hingegen, das inverse Problem so $\mathrm{zu}$ lösen, dass durch die numerische Analyse einer Serie von $n$ Abbildungen mit unterschiedlichen Abbildungsbedingungen mittels statistischer Verfahren die ursprüngliche Objektwelle bestimmt werden kann. Diese Lösung ist aber nicht eindeutig.

- Bei der dritten Gruppe, dem iterativen Bild(serien)vergleich, soll schließlich das inverse Problem gelöst werden, indem man aus der Untersuchung einer Abbildung oder einer Abbildungsserie direkt auf das Streupotential schliefst. Dabei wird versucht, durch den iterativen Vergleich von experimentellen und - auf der Basis eines parametrisierten Streupotentials - simulierten Abbildungen durch entsprechende Anpassungsalgorithmen die optimalen Parameter für das Modell zu bestimmen (s. Abschnitt 2.3).

Eine intuitive, direkte Interpretation elektronenmikroskopischer Abbildungen ist jedoch in der Regel nicht möglich und kann zu falschen Modellen für die der Probe zugrunde liegende Struktur führen. Dafür sind im Wesentlichen drei Gründe verantwortlich:

1. Aufgrund der starken Wechselwirkung zwischen einfallender Elektronenwelle und Materie existiert innerhalb der Probe im allgemeinen Fall Mehrfachstreuung, so dass zwischen dem Probenpotential und der die Probe verlassenden Objektwelle kein linearer Zusammenhang besteht.

2. Die Objektwelle erfährt unterhalb der Probe durch die Objektivlinse eine Modifizierung durch die so genannte kohärente Phasenmodulationsfunktion $T(\vec{q})$ des Mikroskops. Diese sorgt für einen komplexen Zusammenhang zwischen der Objektwelle und der aufzuzeichnenden Bildwelle.

In die beugungswinkelabhängige Funktion $T(\vec{q})$ gehen die Abbildungsfehler der Objektivlinse sowie die Defokussierung des Mikroskops ein:

$$
\begin{aligned}
\widetilde{\psi}_{I}(\vec{q}) & =\widetilde{\psi}_{O}(\vec{q}) \cdot T(\vec{q}) \\
T(\vec{q}) & =\exp \{i \chi(\vec{q})\} \quad \text { mit } \quad \chi(\vec{q})=\pi\left(\frac{C_{s} q^{4} \lambda^{3}}{2}+\Delta f q^{2} \lambda\right)
\end{aligned}
$$

$\widetilde{\psi}_{O}(\vec{q})$ bzw. $\widetilde{\psi}_{I}(\vec{q})$ steht dabei für die Objekt- bzw. Bildwelle im Fourierraum, $\chi(\vec{q})$ für die kohärente Aberrationsfunktion, $C_{S}$ bezeichnet den Öffnungsfehler oder die sphärische Aberration, $\Delta f$ den Defokus, $\lambda$ die Wellenlänge und $\vec{q}$ einen Punkt im reziproken Raum. Neben den beiden genannten Aberrationen gibt es noch weitere Linsenfehler, z.B. den 2- bzw. 3-zähligen Astigmatismus $\left(\sim q^{2}\right.$ bzw. $\left.q^{3}\right)$ oder die axiale Koma $\left(\sim q^{3}\right)$. Diese Aberrationen sind aber bei dem für diese Untersuchung verwendeten Elektronenmikroskop apparativ korrigierbar, so dass ihr Einfluss nach der Korrektur im Vergleich zu dem des Öffnungsfehlers $\left(\sim q^{4}\right)$ klein ist und nicht 

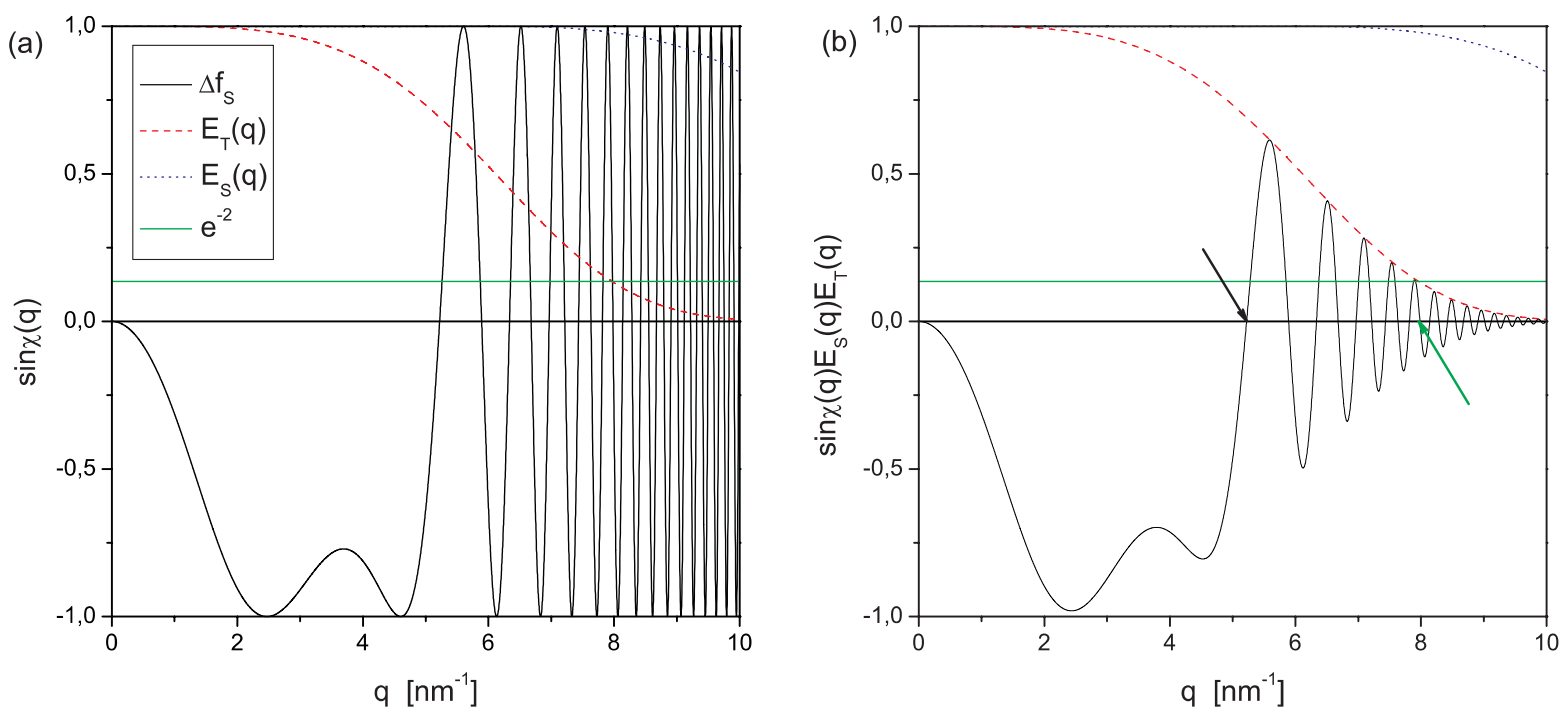

Abbildung 2.2 - (a) Imaginärteil der Phasenmodulationsfunktion für den Scherzer-Fokus $\Delta f_{S}$, sowie die beiden Dämpfungseinhüllenden durch die partielle zeitliche $\left(E_{T}(q)\right.$, rote Kurve) und partielle räumliche $\left(E_{S}(q)\right.$, blaue Kurve) Kohärenz. (b) Imaginärteil der Kontrasttransferfunktion für den Scherzer-Fokus. Bis zur Punktauflösung werden Raumfrequenzen phasengleich abgebildet. Die Punktaufösung ist durch den ersten Nulldurchgang festgelegt (schwarzer Pfeil). Der Wert, bei dem $E_{T}(q)$ auf $e^{-2}$ abgefallen ist, definiert das Informationslimit des Mikroskops (grüner Pfeil).

betrachtet werden muss. Weitere Aberrationen werden erst für höchste Auflösungen wichtig.

Aufgrund der speziellen Form der Phasenmodulationsfunktion ist auch die Punktauflösung kleiner als das Informationslimit ${ }^{\dagger}$ (s. auch Abb. 2.2). Dabei definiert man das Punktaufösungsvermögen des Mikroskops durch den ersten Nulldurchgang des Imaginärteils der Phasenmodulationsfunktion beim so genannten ,Scherzer-Fokus“. In diesem Fokus wird das breiteste Band von Ortsfrequenzen ohne Vorzeichenwechsel und damit ohne Kontrastumkehr übertragen und ist durch

$$
\Delta f_{S}=-\sqrt{1.5 \lambda C_{S}}
$$

festgelegt $t^{\ddagger}$. Für das bei der vorliegenden Untersuchung verwendete Mikroskop

\footnotetext{
${ }^{\dagger}$ Diese Einschränkung gilt nicht für $C_{S}$-korrigierte Elektronenmikroskope oder Hochspannungselektronenmikroskope, für die Punkt- gleich Informationslimit ist.

${ }^{\ddagger}$ Streng genommen ist der Scherzer-Fokus durch $\Delta f_{S}=-\sqrt{\lambda C_{S}}$ definiert ${ }^{[45]}$. Nimmt man in Kauf, dass sich die Kastenform im mittleren Bereich durch eine Einbuchtung verschlechtert (s. Abb. 2.2), kann durch die Verwendung des durch Gleichung 2.3 festgelegten Wertes die Punktauflösung jedoch zu einer höheren Raumfrequenz verschoben werden. Dieser Fokus wird in der Literatur außer ScherzerFokus auch als „optimaler Defokus“ bezeichnet.
} 
CM200-UT-FEG der Firma Philips ergibt sich bei einer Beschleunigungsspannung von $U_{B}=200 \mathrm{kV}(\lambda=2.51 \mathrm{pm})$ sowie einem Öffnungsfehler von $C_{S}=0.48 \mathrm{~mm}$ ein Scherzer-Fokus von $\Delta f_{S}=-42.5 \mathrm{~nm}$ und eine Punktauflösung von $d_{P}=1.9 \AA$ $\left(q_{P}=5.3 \mathrm{~nm}^{-1}\right)$. Die Defokussierung der Objektivlinse im Falle der „Unterfokussierung" wird mit negativen Werten angezeigt. Dabei bezeichnet Unterfokussierung die Fokussierung der Objektivlinse auf eine Ebene unterhalb der Probe.

Im Gegensatz zu den eben diskutierten kohärenten Aberrationen existieren auch inkohärente, also Informationen zerstörende Aberrationen. Diese bestimmen das Informationslimit des Mikroskops und haben ihren Ursprung in der partiellen zeitlichen (Schwankung des Defokus) und partiellen räumlichen (endliche Konvergenz der Elektronenwelle) Kohärenz der Elektronenwelle. Beide Effekte $\left(E_{T}, E_{S}\right)$ sorgen im einfachsten Fall jeweils für eine Dämpfungseinhüllende (vgl. Abb. 2.2). Da die partielle räumliche Kohärenz von der Wahl des Defokus abhängt, wird das Informationslimit allein durch die partielle zeitliche Kohärenz bestimmt, welche nur von den Eigenschaften des Mikroskops beeinflusst wird. Der Wert, bei dem dessen Dämpfungseinhüllende auf $e^{-2} \approx 13 \%$ abgefallen ist, definiert das Informationslimit und beträgt für das CM200: $d_{\text {info }}=1.26 \AA\left(q_{\text {info }} \approx 8 \mathrm{~nm}^{-1}\right)$.

Das Produkt der Dämpfungseinhüllenden mit der Phasenmodulationsfunktion wird auch als Kontrasttransferfunktion „CTF“ („Contrast Transfer Function“) bezeichnet.

$$
\operatorname{CTF}(\vec{q})=E_{S}(\vec{q}) \cdot E_{T}(\vec{q}) \cdot T(\vec{q})
$$

Streng genommen gehört zu der Kontrasttransferfunktion auch noch eine Aperturfunktion, welche alle Raumfrequenzen größer als $\vec{q}_{\text {Aper }}$ entsprechend der gewählten Apertur abschneidet.

Die kombinierte Wirkung von Öffnungsfehler der Objektivlinse und Defokus führt im Realraum dazu, dass jeder Punkt der Probe in der Abbildung in einen ausgedehnten Bereich transformiert wird. Diese „Delokalisierung“ ist raumfrequenzabhängig. Sie wird durch den Radius der Punktaufweitungsfunktion quantifiziert ${ }^{[46]}$.

$$
R_{\text {Delok }}=\max \left|\frac{\partial \chi(\vec{q})}{\partial \vec{q}}\right| ; \quad \text { für } \vec{q} \in\left[0, \vec{q}_{\max }\right]
$$

Dieser Effekt läßt sich gut am Beispiel eines schwachen Phasenobjekts veranschaulichen, bei dem die Elektronenwelle beim Durchlaufen einer Probe lediglich eine kleine räumliche Variation der Phase erfährt. Abbildung 2.3 zeigt für drei verschiedene Defoki jeweils auf der linken Seite den Imaginärteil der Kontrasttransferfunktion und auf der rechten Seite die korrespondierende Punktaufweitungsfunktion im Realraum, welche in diesem Fall die Fouriertransformierte von $\sin \chi(q)$ ist ${ }^{[47]}$. Es ist deutlich erkennbar, dass sich die Intensität in Abhängigkeit vom Defokus 

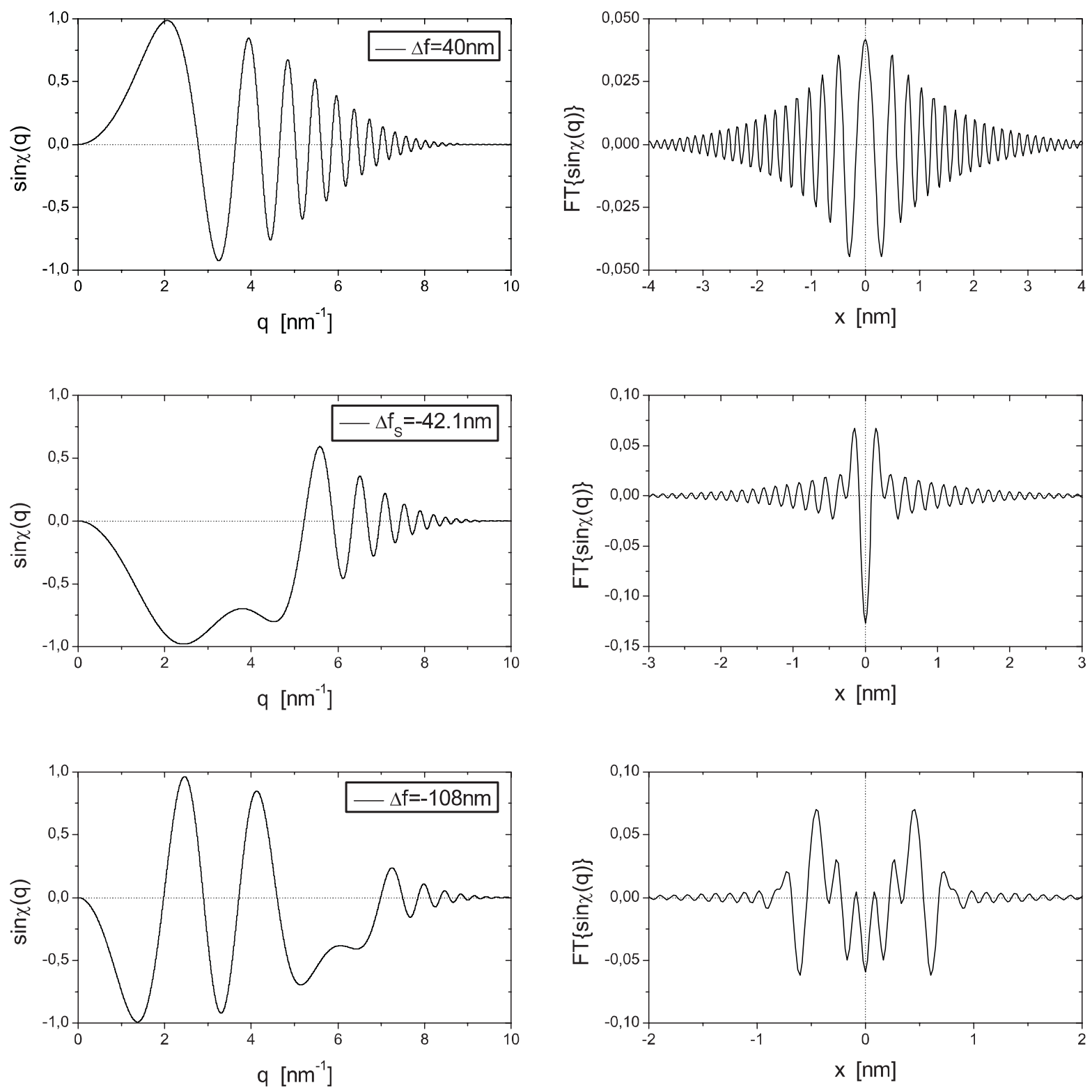

Abbildung 2.3 - Imaginärteil der Kontrasttransferfunktion und Punktaufweitungsfunktion. Auf der linken Seite ist für verschiedene Defokussierungen der Imaginärteil der Kontrasttransferfunktion und auf der rechten Seite jeweils die korrespondierende Punktaufweitungsfunktion dargestellt. 
z.T. über mehrere Nanometer weit und in komplizierter Art und Weise auf die Umgebung verteilt. Die Ausdehnung der Delokalisierung liegt damit im Bereich der abzubildenen Abstände und erschwert die direkte Interpretation von elektronenmikroskopischen Abbildungen erheblich.

3. Als letzter Grund, weshalb elektronenmikroskopische Abbildungen in der Regel nicht direkt interpretiert werden können, ist abschließend noch kurz der Verlust der Phaseninformation der Bildwelle beim Aufzeichnungsprozess zu nennen.

\subsubsection{Kartierungsverfahren}

Alle Kartierungsverfahren versuchen, strukturelle oder chemische Veränderungen in der Probe durch die Informationen, die in den elektronenmikroskopischen Abbildungen enthalten sind, zu bestimmen und innerhalb der Abbildung darzustellen. All diesen Verfahren ist gemein, dass die Intensitätsverteilung lokal mit der zu untersuchenden Eigenschaft (Dicke, chemische Zusammensetzung, Gitterverzerrung) in Verbindung stehen muss. Zu diesem Zweck wird z.B. bei der „QUANTITEM“-Methode ${ }^{[48-51]}$ („QUantitative ANalysis of The Information from Transmission Electron Micrographs") die Abbildung in Bildeinheitszellen unterteilt und die Intensitätsverteilungen innerhalb dieser Zellen, im Folgenden „Muster“ genannt, untersucht. Unter bestimmten Bedingungen können Variationen dieser Muster in eine relative Änderung der Probendicke umgerechnet werden. Zwei weitere Kartierungsverfahren, das „,chemical mapping“ [52,53] und die „CELFA“Analyse $^{[54]}$ („Composition Evaluation by Lattice Fringe Analysis“), bestimmen die lokale chemische Zusammensetzung einer Probe, indem sie die Veränderungen in den Mustern untersuchen, welche auf Überstrukturreflexe und nicht auf Dickenvariationen zurückzuführen sind. Solche Reflexe entstehen, wenn die chemische Zusammensetzung der die Untergitter einer Struktur besetzenden Atomsorte unterschiedlich ist. Gitterverzerrungen dagegen können durch das so genannte ,strain mapping“ “55-61] quantifiziert werden. Dabei werden die Positionen der Intensitätsmaxima analysiert, welche wiederum unter bestimmten Abbildungsbedingungen die projizierten Atomsäulen repräsentieren. Die Auswertung der Abstände zwischen den Intensitätsmaxima ergibt schließlich die lokale Verzerrung des zugrunde liegenden Gitters. In diesem Fall ist keine Unterteilung der Abbildung in Bildeinheitszellen nötig.

\subsubsection{Untersuchung der Objektwelle}

Die Objektwelle kann entweder aus einer gemessenen Bildwelle (Holographie-Technik ${ }^{[62,63]}$ ) oder aus einer gemessenen Defokusserie rekonstruiert werden (Paraboloid „PAM" Methode ${ }^{[64-68]}$, maximum-likelihood „MAL“ Algorithmus $\left.{ }^{[69-71]}\right)$. Der Vorteil der Objektwelle liegt darin, dass sie Informationen bis zum Informationslimit des Mikroskops enthält und 
im Gegensatz zur Bildwelle nicht von den Aberrationen der Objektivlinse beeinflusst ist (s. auch Abschnitt 2.2.1).

Bei der Holographie-Technik wird der Elektronenstrahl sowohl durch die Probe (Objektwelle) als auch daran vorbei (Referenzwelle) geführt und mittels eines Möllenstedtschen Biprismas, welches sich zwischen der hinteren Brennebene und der Bildebene befindet, ein Interferenzmuster zwischen beiden Wellen aufgezeichnet. Dieses Hologramm ermöglicht die Bestimmung der komplexwertigen Bildwelle, die um die kohärenten Abbildungsfehler korrigiert werden kann und somit die Rekonstruktion der Objektwelle auf numerischem Wege erlaubt.

Im Gegensatz dazu wird für den Rekonstruktionsansatz eine „Defokusserie“ aufgezeichnet, d.h. eine Serie von Aufnahmen mit unterschiedlicher Defokussierung, also unterschiedlichen Abbildungsbedingungen des Mikroskops. Durch dieses Vorgehen können die Effekte des Abbildungsprozesses durch numerische Rekonstruktion von der Wellenfunktion getrennt werden.

Die starke Wechselwirkung der die Probe durchlaufenden Elektronen mit den Atompotentialen resultiert jedoch in dynamischer Vielfachstreuung, was, wie bereits in Abschnitt 2.2.1 erwähnt, zu einem komplexen Zusammenhang zwischen dem projizierten Probenpotential und der Objektwelle führt. Dies gilt in besonderem Maße für dicke Proben mit schweren Elementen und für kristalline Materialien, welche entlang einer niedrig indizierten Zonenachse orientiert sind. Methoden zur Rekonstruktion des projizierten Potentials sind aber vorhanden, vgl. z.B. die Methode von Lentzen ET AL. auf der Basis eines „,maximum-likelihood"-Algorithmus ${ }^{[72]}$.

Für den Fall, dass die Wechselwirkung zwischen Elektronenwelle und Probenpotential im Wesentlichen zu einer Phasenschiebung führt (Phasenobjekt), kann schon die Phase der Objektwelle Aufschluss über die Struktur der Probe geben, wie verschiedene Untersuchungen gezeigt haben ${ }^{[73-76]}$.

\subsection{Iterative Anpassung von Bildserien}

Das für diese Untersuchung angewendete Verfahren des iterativen Vergleichs von Bildserien soll in den folgenden Abschnitten erklärt werden. Dabei wird in Abschnitt 2.3.1 die Grundidee der Methode beschrieben, während in den Abschnitten 2.3.2 und 2.3.3 die Implementation von MöBUS ET AL. ${ }^{[8,9]}$ und die praktischen und prinzipiellen Probleme bei der Durchführung erläutert werden. Abschließend sollen in Abschnitt 2.3.4 die Unterschiede zu der in dieser Arbeit verwendeten Vorgehensweise kurz aufgezeigt werden. 


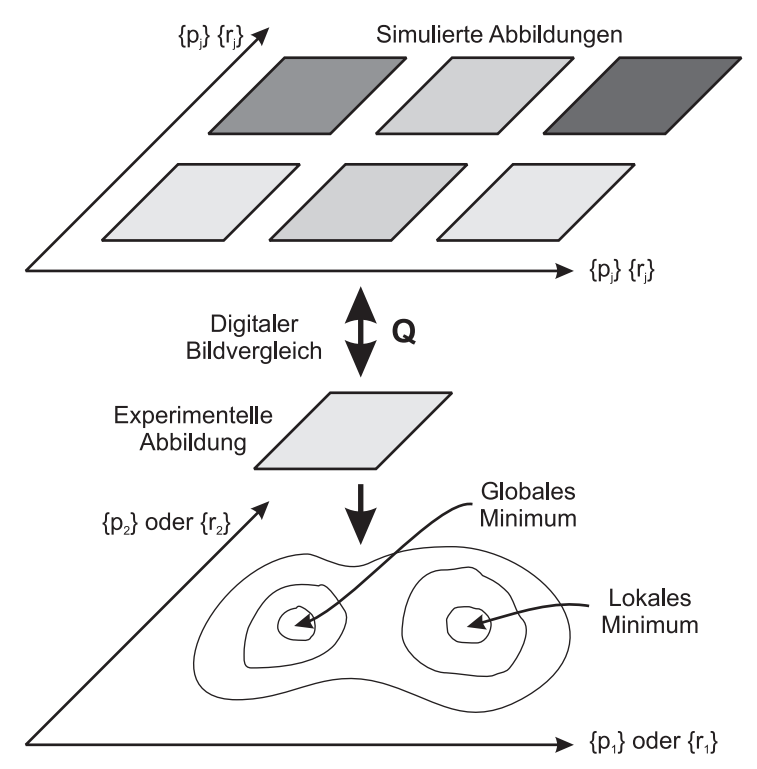

Abbildung 2.4 - Schematische Beschreibung der Methode des iterativen Bildvergleichs. Für diverse mögliche Strukturmodelle werden elektronenmikroskopische Abbildungen simuliert und mittels eines Bildvergleichsmaßes $Q$ mit der experimentellen Abbildung quantitativ verglichen. Die Auswertung der Verteilung der Anpassungswerte ergibt schließlich den besten Parametersatz für das Strukturmodell. $p_{i}$ bezeichnet die Simulationsparameter (z.B. Defokus, Probendicke) und $r_{j}$ die Strukturparameter (Positionen der Atomsäulen). Die Abbildung ist der Arbeit von MöBus ET AL. ${ }^{[9]}$ entnommen.

\subsubsection{Die Grundidee}

Das Verfahren des iterativen Vergleichs von elektronenmikroskopischen Abbildungen besteht in dem quantitativen Vergleich von Intensitätsverteilungen experimenteller und simulierter Abbildungen. Abbildung 2.4 illustriert diese Methode graphisch. Dabei werden elektronenmikroskopische Abbildungen auf der Basis von parametrisierten Strukturmodellen mittels eines Simulationsprogramms, z.B. des EMS-Pakets von STADELMANN $^{[77]}$, simuliert und mit der experimentellen Aufnahme quantitativ verglichen. Da elektronenmikroskopische Abbildungen meistens entlang Richtungen hoher Symmetrie aufgenommen werden, besteht die Parametrisierung der Struktur im Wesentlichen in der Positionierung von Atomsäulen entlang des Elektronenstrahls. Die besten Parameter des Modells werden schließlich in einem iterativen Prozeß durch Optimierung eines Bildvergleichsmaßes bestimmt. Das Ergebnis ist eine mögliche strukturelle Realisierung der untersuchten Probe. Abgeschlossen wird die Methode des iterativen Bildvergleichs, indem man die Genauigkeit der bestimmten Parameter abschätzt. 


\subsubsection{Das Standardverfahren nach Möbus}

Die Standardvorgehensweise beim iterativen Bildvergleich stellt die „IDIM“-Methode nach MöBus ${ }^{[8,9]}$ (,Iterative Digital Image Matching") dar. Im Folgenden sollen die vier Schritte dieser Implementation kurz beschrieben werden:

1. Vorbehandlung der Abbildung: Diesem Schritt kommt besondere Bedeutung zu, da die Berechnung eines Bildvergleichsmaßes nur dann sinnvoll ist, wenn experimentelle und simulierte Abbildungen sowohl in ihrer relativen Position als auch in den Intensitäten übereinstimmen. Dabei ist das Ausrichten der Abbildungen nicht weiter schwierig und kann z.B. durch das Kreuzkorrelationsverfahren ${ }^{[29,78]}$ oder anhand der Phasenkorrelationsfunktion ${ }^{[79]}$ erfolgen. Ein fundamentales Problem beim quantitativen Intensitätsvergleich besteht jedoch in dem großen Unterschied zwischen experimentellen und simulierten Kontrasten. Dieser Unterschied ist in

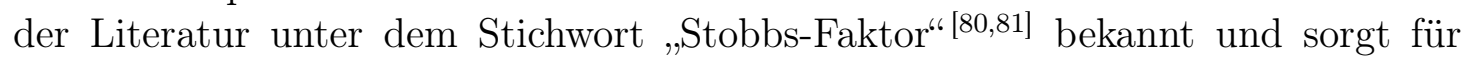
einen systematischen Fehler im quantitativen Vergleich (s. auch Abschnitt 2.3.3). MÖBUS ET AL. versuchen dieses Problem durch entsprechende Normierungen der Abbildungen zu umgehen ${ }^{[8]}$. So werden z.B. bei der Standardisierung der Abbildungen sowohl die mittlere Intensität als auch die Standardabweichungen in der experimentellen und simulierten Abbildung gleichgesetzt:

$$
\begin{aligned}
I^{\prime}(x, y) & =\frac{I(x, y)-\bar{I}}{\sigma} \\
\operatorname{mit} \sigma^{2} & =\left\langle(I(x, y)-\bar{I})^{2}\right\rangle
\end{aligned}
$$

Hierbei bezeichnet $I(x, y)$ die Intensitätsverteilung, $\bar{I}$ die mittlere Intensität und $\sigma^{2}$ den Kontrast in der experimentellen bzw. simulierten Abbildung. Weitere Normierungsmöglichkeiten sind bei MÖBUS ET AL. ${ }^{[8]} \mathrm{zu}$ finden.

2. Simulation und Vergleich: Aufgrund der oben genannten Kontrastunterschiede ist auch die Wahl des Bildvergleichsmaßes nicht universell, sondern muss der zu untersuchenden Fragestellung angepasst werden. So führen verschiedene Vergleichsmaße unter Umständen zu unterschiedlichen Modellstrukturen ${ }^{[82]}$. Des Weiteren übt die Normierung ebenfalls einen Einfluss auf die Wahl des Bildvergleichsmaßes aus. Eine Auflistung verschiedener Maße sind bei MöBus ET AL. ${ }^{[8,9,82]}$ und HILLEBRAND ET AL. ${ }^{[83]} \mathrm{zu}$ finden. Drei der am häufigsten benutzten Bildvergleichsmaße sind die Kreuzkorrelation (xcf), das euklidische Abstandsmaß (ed) und der 
R-Faktor (R):

$$
\begin{aligned}
\operatorname{xcf}(E, S) & =\frac{\sum_{x, y=1}^{n x, n y}(E(x, y)-\bar{E}) \cdot(S(x, y)-\bar{S})}{\sqrt{\sum_{n x, y=1}^{n x, n y}(E(x, y)-\bar{E})^{2} \cdot \sum_{x, y=1}^{n x, n y}(S(x, y)-\bar{S})^{2}}} \\
\operatorname{ed}(E, S)^{2} & =\sum_{x, y=1}^{n, n y}(E(x, y)-S(x, y))^{2} \\
\mathrm{R}(E, S) & =\frac{\sum_{x, y=1}^{n x, n y}(E(x, y)-S(x, y))^{2}}{|E|^{2}}
\end{aligned}
$$

Dabei steht $E$ bzw. $S$ jeweils für die Intensitätsverteilung der experimentellen bzw. simulierten Abbildung, $\bar{E}$ bzw. $\bar{S}$ für die entsprechende mittlere Intensität und $(x, y)$ bezeichnen die Bildpunkte, für die $1 \leq x \leq n x$ bzw. $1 \leq y \leq n y$ gilt.

Die Kreuzkorrelation ist gut geeignet für den Fall, dass die mittlere Intensität der Abbildungen nicht interpretierbar und der Kontrast in der Simulation und dem Experiment stark unterschiedlich ist, da lediglich die Muster miteinander verglichen werden. Der euklidische Abstand hingegen bewertet die gesamte Information der Intensitätsverteilung in der experimentellen Abbildung. Der R-Faktor schließlich ist aus der Analyse von Röntgenbeugungsmustern bekannt ${ }^{[8]}$. Er normiert den euklidischen Abstand auf die mittlere Intensität der experimentellen Abbildung.

Die Simulation der elektronenmikroskopischen Abbildungen erfolgt, wie bereits in Abschnitt 2.3.1 erwähnt, auf der Basis parametrisierter Strukturmodelle mittels eines Simulationsprogramms.

3. Optimierung: Für den Optimierungsprozess der Parameter des Strukturmodells können diverse numerische Methoden verwendet werden. Einen Überblick geben MÖBUS ET AL. ${ }^{[9]}$.

4. Fehlerbetrachtung: Die Angabe von Atompositionen bzw. Positionen von Atomsäulen am Ende des Optimierungsprozesses ist nur sinnvoll, wenn sie mit der Angabe eines Fehlers verknüpft ist. In erster Linie tragen zu solchen Fehlern experimentelle Ungenauigkeiten bei. Vor allem ist auf eine saubere Probe, genaue Kenntnis der Parameter des Elektronenmikroskops sowie dessen Einstellung zu achten. Eingehendere Behandlungen zur Abschätzung der Konfidenz sind in der entsprechenden Literatur ${ }^{[82,84-88]} \mathrm{zu}$ finden.

\subsubsection{Der Stobbs-Faktor}

Der Kontrast in experimentellen Abbildungen ist typischerweise um den Faktor drei bis fünf geringer als in den simulierten, so dass ein quantitativer Vergleich von Intensitäten 
ohne vorhergehende Normierung nicht sinnvoll ist (s. Abschnitt 2.3.2). Die Ursache des so genannten „,Stobbs-Faktors “ ${ }^{[80,81]}$ ist Gegenstand aktueller Forschung ${ }^{[89-96]}$. Im Folgenden soll der aktuelle Wissensstand kurz referiert werden.

Es existiert eine Vielzahl von Effekten, die zu einer Reduzierung des Kontrasts führen, jedoch in den herkömmlichen Simulationsprogrammen keine Berücksichtigung finden. Zum Teil können sie aber vor bzw. nach der Simulation einbezogen werden. Dabei handelt es sich insbesondere um die Übertragungseigenschaften des Aufzeichnungsmediums und die Probendrift. Weitere Effekte sind z.B. Probenvibrationen, Phononenstreuung oder auch Unregelmäßigkeiten der Probe selbst, wie z.B. Verspannungen, Defekte oder amorphe Deckschichten. Einen Überblick über diese Effekte gibt BoOTHROYD ${ }^{[91]}$.

Dabei ist der Kontrastunterschied nicht auf kristalline Materialien beschränkt, er findet sich über einen weiten Frequenzbereich sowohl in Abbildungen sehr dünner ${ }^{[92]}$ als auch dicker amorpher Proben ${ }^{[91,93]}$. Ein solches Verhalten spricht z.B. gegen Probenvibrationen, da in diesem Fall die hohen Raumfrequenzen stärker gedämpft sein müssten als die niedrigen. Es deutet vielmehr auf eine zusätzliche konstante Hintergrundintensität hin, welche typischerweise in thermisch diffuser Streuung, also quasi-elastischer Streuung durch die thermische Bewegung der Atome oder Phononenstreuung, ihre Ursache hat. Diese Annahme wird unterstuitzt durch die Tatsache, dass sich der Kontrastunterschied auch in energiegefilterten Abbildungen findet und somit alle inelastischen Streuprozesse als Hauptursache ausgeschlossen werden können. Deswegen konzentriert sich die aktuelle Forschung derzeit auf die Holographie-Technik, zumal HARSCHER ${ }^{[97]}$ und WANG ${ }^{[98]}$ gezeigt haben, dass in den Abbildungen aus den Seitenbändern im Gegensatz zum Zentralband die Beiträge thermisch diffuser Streuung keine Rolle spielen. LEHMANN ET AL. ${ }^{[94]}$ konnten mittlerweile experimentell nachweisen, dass der Kontrast in den Abbildungen aus den Nebenbändern um den Faktor drei bis fünf größer ist als in der Abbildung aus dem Zentralband. Parallel dazu hat BooTHROYD ${ }^{[95]}$ jedoch anhand von Untersuchungen der Hintergrundintensität von energiegefilterten Beugungsmustern gezeigt, dass der Anteil thermisch diffus gestreuter Elektronen nicht ausreicht, um den gesamten Kontrastunterschied in den Gitterabbildungen zu erklären.

\subsubsection{Anpassung von Bildserien}

In der vorliegenden Untersuchung wurde das im Abschnitt 2.3.2 beschriebene Standardverfahren nach MöBus auf die Anpassung von Bildserien erweitert. Dies bedeutet, dass mehrere Abbildungen mit unterschiedlichen Abbildungsbedingungen, d.h. unterschiedlicher Defokussierung des Mikroskops, aufgenommen werden und die gesamte Defokusserie simultan mit Simulationen verglichen wird. Diese Vorgehensweise hat zwei Vorteile: Zum einen bewirkt die größere Anzahl experimenteller Abbildungen eine Erhöhung der Konfidenz in die angepassten Parameter des Strukturmodells. Zum anderen wird der Effekt der Delokalisierung (s. Abschnitt 2.2.1) bei der Strukturbestimmung zuverlässig von dem der grenzflächeninduzierten Ordnung getrennt, während dies bei der Anpassung 
einer einzelnen Abbildung noch zu falschen Strukturmodellen führen kann ${ }^{\S}$.

Die Details der Implementation werden in Kapitel 3 ausführlich dargestellt.

\subsection{Hochauflösende Elektronenmikroskopie an amorph/kristallinen Grenzflächen}

Aufgrund ihrer exponierten Stellung innerhalb der Halbleitertechnologie wurden an der amorph/kristallinen Grenzfläche zwischen Silizium und amorphem Siliziumdioxid bereits vielfach Untersuchungen durchgeführt. Um einen Überblick zu geben, wie stark die bestimmten Strukturen und deren Interpretation von der benutzten Analysetechnik abhängen und sich voneinander unterscheiden, sollen die Ergebnisse dieser Forschungen im Folgenden kurz zusammengefasst werden.

OURMAZD ET AL. ${ }^{[99]}$ wuchsen eine thermisch oxidierte Schicht $\mathrm{SiO}_{2}$ auf $\mathrm{Si}(100)$ auf. Durch Vergleiche der experimentellen Gitterabbildungen in direkter Nähe der Grenzfläche mit simulierten Abbildungen schlossen sie auf epitaktisches Wachstum einer kristallinen, aber verspannten $\mathrm{SiO}_{2}$-Schicht, welche als „Tridymit"-Phase identifiziert wurde und eine stabile Phase des $\mathrm{SiO}_{2}$ mit hexagonaler Struktur $(a=5.046 \AA, c=8.23 \AA)$ darstellt. Dabei wuchsen die ersten zwei Atomlagen perfekt kristallin auf, während das $\mathrm{SiO}_{2}$ über die folgenden drei Atomlagen komplett amorphisierte. Auf demselben Weg fanden IKARASHI ET AL. ${ }^{[100]}$ zwar auch eine epitaktisch gewachsene kristalline $\mathrm{SiO}_{2}$-Lage an der $\mathrm{SiO}_{2} / \mathrm{Si}(001)-G r e n z f l a ̈ c h e$, allerdings erklärten sie den Kontrast durch die Existenz einer „Kristoballit"-Phase. Diese ist genauso wie das Tridymit eine stabile Phase des $\mathrm{SiO}_{2}$, weist jedoch dieselbe Struktur wie Silizium auf, nur dass zwischen den Si-Si-Bindungen Sauerstoff eingefügt ist. Dies wiederum resultiert in einer $40 \%$ größeren Gitterkonstanten $(a=7.6 \AA)$. Aus eben diesem Grund schlossen OuRmazD ET AL. ${ }^{[99]}$ auch die Existenz einer epitaktisch gewachsenen Kristoballit-Lage aus, da ein solches Wachstum zu einer unrealistisch großen Verzerrung der Bindungswinkel an der Grenzfläche führen würde. Auf der anderen Seite hingegen konnten IKARASHI ET AL. ${ }^{[100]}$ den von ihnen gemessenen Grenzflächenkontrast nicht auf der Basis einer Tridymit-Phase simulieren. Außerdem wird für das Wachstum der Tridymit-Lage eine atomar glatte $\mathrm{Si}(001)$-Oberfläche benötigt, welche in ihren Experimenten nicht nachgewiesen werden konnte.

Im Gegensatz zu diesen Autoren fanden AKATSU ET AL. ${ }^{[101]}$ durch den kombinierten Vergleich von elektronenmikroskopischen Mustern an der Grenzfläche sowie des Beugungsmusters mit Simulationen eine (111)-facettierte Grenzfläche. Im Unterschied zu der herkömmlichen Vorgehensweise einer elektronenmikroskopischen Untersuchung, bei der dünne Proben untersucht werden, legten AKATsU ET AL. Wert auf die Untersuchung dicker Proben, da sie vermuteten, dass sich die Grenzflächenmorphologie bei der

${ }^{\S}$ Der Effekt der Delokalisierung kann durch die Verwendung eines $C_{S}$-korrigierten Elektronenmikroskops oder eines Hochspannungselektronenmikroskops aber minimiert werden. 
Herstellung dünner Proben durch die üblicherweise benutzte Ionendünnung modifizieren könnte. Die Hypothese von der Existenz einer epitaktisch gewachsenen Tridymit-Lage wurde auch durch das Vorhandensein einer rauhen Grenzfläche ausgeschlossen.

Im Gegensatz zu diesen drei Veröffentlichungen maßen NAKANISHI ET AL. ${ }^{[102]}$ keine Veränderung der atomaren Struktur an der Grenzfläche und schlossen die Existenz sowohl einer Tridymit- als auch einer Kristoballit-Phase aus. Sie interpretierten kristallinen Kontrast an der Grenzfläche als kristallines Silizium, welches in das amorphe $\mathrm{SiO}_{2}$ maximal 4 Monolagen hineinragt. Diese Untersuchung wurde mittels der „HAADF STEM“-Technik („High Angle Annular Dark Field Scanning Transmission Electron Microscopy") durchgeführt, welche im Gegensatz zu der hochauflösenden Elektronenmikroskopie eine inkohärente Aufnahmetechnik ist, d.h. dass die Elektronen, welche aufgrund inkohärenter Streuprozesse unter einem hohem Winkel aus der Probe herausgestreut werden, detektiert werden.

Wiederum einen anderen Weg schlugen OHDOMARI ET AL. ${ }^{[103]}$ ein. Sie simulierten unter Zuhilfenahme eines per Hand erstellten Netzwerkmodells die elektronenmikroskopische Grenzflächenabbildung zwischen $\mathrm{SiO}_{2} / \mathrm{Si}(111)$ und fanden eine scheinbare Verschiebung des kristallinen Kontrasts relativ zur Position der Grenzfläche um eine Atomlage in das amorphe Material hinein. Dies erklärten sie durch einen ordnenden Effekt der aus der $\mathrm{Si}(111)$-Oberfläche senkrecht herausragenden, mit Sauerstoff verbundenen Si[111]-Bindungen. Durch den festen Bindungswinkel sind die möglichen Positionen für die folgenden Siliziumatome auf einen kleinen Bereich eingeschränkt. Im Gegensatz zu den anderen Ansätzen beinhaltet diese Vorgehensweise erstmals mögliche Atompositionen des amorphen Materials, welches sonst in die Simulationen als Vakuum eingeht.

Zusammenfassend muss festgestellt werden, dass - außer bei OHDOMARI ET AL. - eine angemessene Berücksichtigung der atomaren Struktur des amorphen Teils der Grenzfläche fehlt, so dass große Unsicherheiten bei der Strukturbestimmung auftreten, die wiederum zu unterschiedlichen Interpretationen führen. 


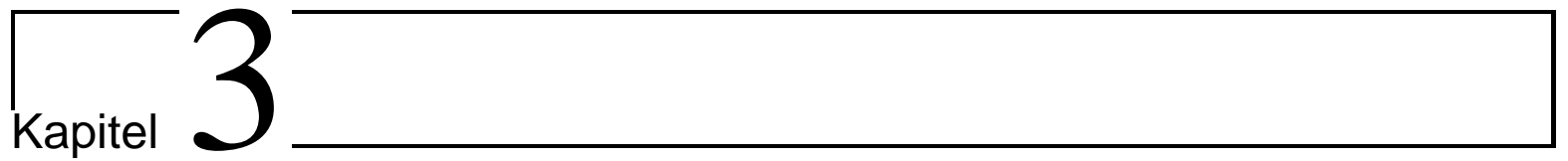

\section{Quantitative HREM an amorph/kristallinen Grenzflächen}

\section{Inhaltsangabe}

3.1 Elektronenmikroskopische Kontraste an der a/c Grenzfläche 24

3.2 Iterativer Bildvergleich gemittelter Grenzflächenabbildungen einer Defokusserie . . . . . . . . . . . 26

3.2.1 Berechnung gemittelter experimenteller Grenzflächenabbildungen . . . . . . . . . . . . . . . 26

3.2.2 Multislice-Simulation gemittelter Abbildungen . . . . . . . . . . 30

3.2.3 Vergleich simulierter und experimenteller Defokusserien . . . . 40

\subsection{Untersuchung der strukturellen Homogenität entlang der} Grenzfläche ................. 42

3.3.1 Die laterale Ausdehnung des Mittelungsbereiches . . . . . . . . 43

3.3 .2 Fehlerbetrachtung . . . . . . . . . . . . 46

In diesem Kapitel wird beschrieben, wie durch den iterativen Bildvergleich zwischen experimentellen und simulierten Defokusserien strukturelle Details der Grenzflächenstruktur zwischen amorphen und kristallinen Materialien bestimmt werden können. Zu diesem Zweck wird in Abschnitt 3.1 zunächst die dem Auswertungsverfahren zugrunde liegende Modellvorstellung des strukturellen Übergangs und dessen Einfluss auf die elektronenmikroskopischen Abbildungen kurz erläutert. Der darauffolgende Abschnitt 3.2 beschreibt die genaue Vorgehensweise beim iterativen Bildvergleich gemittelter Grenzflächenabbildungen. Dabei befasst sich der Abschnitt 3.2.1 mit der Berechnung und Korrektur der Probendrift sowie der Bestimmung gemittelter experimenteller Grenzflächenabbildungen. Während der erste Schritt sicherstellt, dass über die gesamte Defokusserie immer derselbe Probenbereich ausgewertet wird, ist der zweite für die in dieser Untersuchung verwendete statistische Beschreibung des strukturellen Grenzflächenüber- 
gangs nötig. Abschnitt 3.2.2 zeigt, wie die experimentellen gemittelten Grenzflächenabbildungen simuliert werden, und erklärt das für den strukturellen Übergang verwendete Modell, welches in die Simulationen eingeht. Schließlich befasst sich Abschnitt 3.2.3 mit dem quantitativen Vergleich zwischen Experiment und Simulation. Den Abschluß dieses Kapitels bildet mit Abschnitt 3.3 die Beschreibung, wie die quantitative strukturelle Untersuchung entlang der Grenzfläche durchgeführt und auf welche Weise eine Fehlerabschätzung der Modellparameter erreicht wird.

\subsection{Elektronenmikroskopische Kontraste an der amorph/kristallinen Grenzfläche}

Alle in dieser Arbeit untersuchten Proben wurden in Querschnittsgeometrie abgebildet. Die Abbildungen 3.1(a) und (b) zeigen jeweils einen Ausschnitt einer Grenzflächenabbildung der a-Ge/c-Si- bzw. a-Si/c-Si-Probe.

Da das kristalline Material durch seine periodische Kristallstruktur ausgezeichnet ist, ergibt sich auf der kristallinen Seite entsprechend der abgebildeten Struktur und Orientierung der Probe ein kristallperiodischer Kontrast. Dabei soll davon abgesehen werden, dass dieser durch z.B. amorphe Deckschichten der Probe, Dickenvariationen oder lokale Probenverbiegungen über dem abgebildeten Probenbereich variieren kann.

Da das amorphe Material keine langreichweitigen strukturellen Korrelationen aufweist, sind diese auch in den elektronenmikroskopischen Abbildungen nicht zu sehen. Dies wird durch die Autokorrelationsfunktion bestätigt, welche mit zunehmendem Abstand im Gegensatz zu der des kristallinen Materials schnell abfällt.

Aufgrund der stark gerichteten kovalenten Bindung der ersten ,amorphen“ Atome an die letzten dem Kristall zugeschriebenen streng periodisch angeordneten Atome ist im Grenzbereich mit einer grenzflächeninduzierten kristallperiodischen Ordnung zu rechnen, die mit zunehmender Entfernung von der Grenzfläche abnimmt. Diese langreichweitigen Korrelationen führen im Gegensatz zu dem rein amorphen Material wiederum zu kristallperiodischen Kontrasten an der Grenzfläche.

Eine weitere Ursache für langreichweitige Korrelationen in den Kontrasten im grenzflächennahen Bereich, die nicht strukturellen Ursprungs sind, ist die defokusabhängige Delokalisierung der Bildintensität (s. auch Abschnitt 2.2.1). Dieser Effekt muss bei der Strukturbestimmung von dem der grenzflächeninduzierten Ordnung getrennt werden. Dies kann entweder durch die Untersuchung der Objektwellenfunktion selbst geschehen oder, wie in dieser Arbeit, durch die simultane Untersuchung mehrerer Abbildungen mit unterschiedlicher Defokussierung erreicht werden.

Um die kristallperiodischen Anteile aus den experimentellen Abbildungen zu extrahieren, wurden die Abbildungen entlang der Grenzfläche gemittelt, wie später in Abschnitt 3.2.1.2 gezeigt wird. Dabei muss allerdings sichergestellt sein, dass bei der Mittelung 
(a)
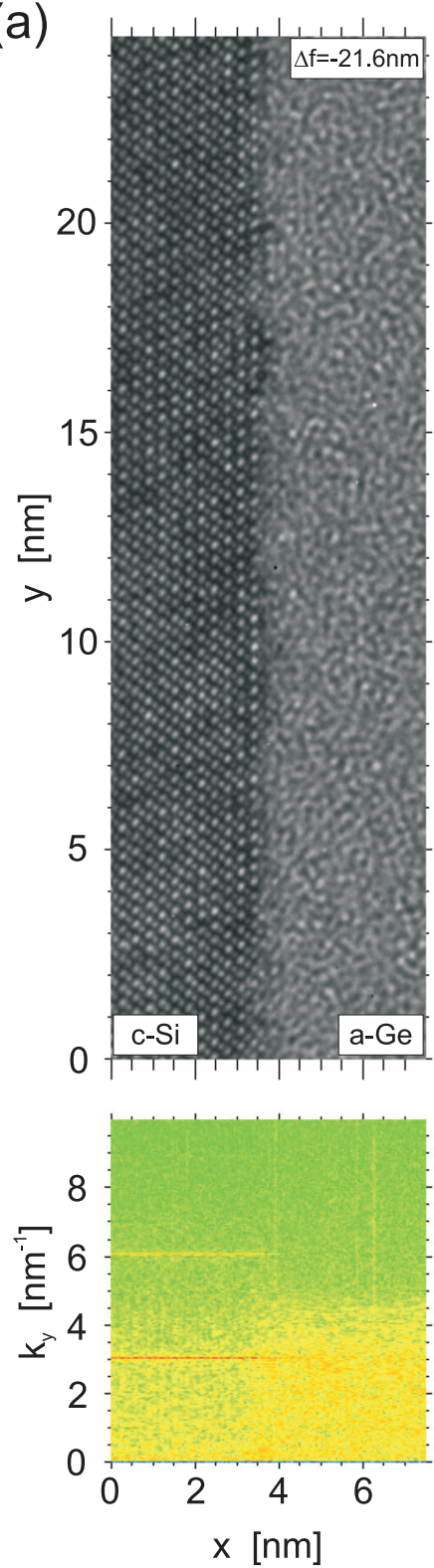

(b)
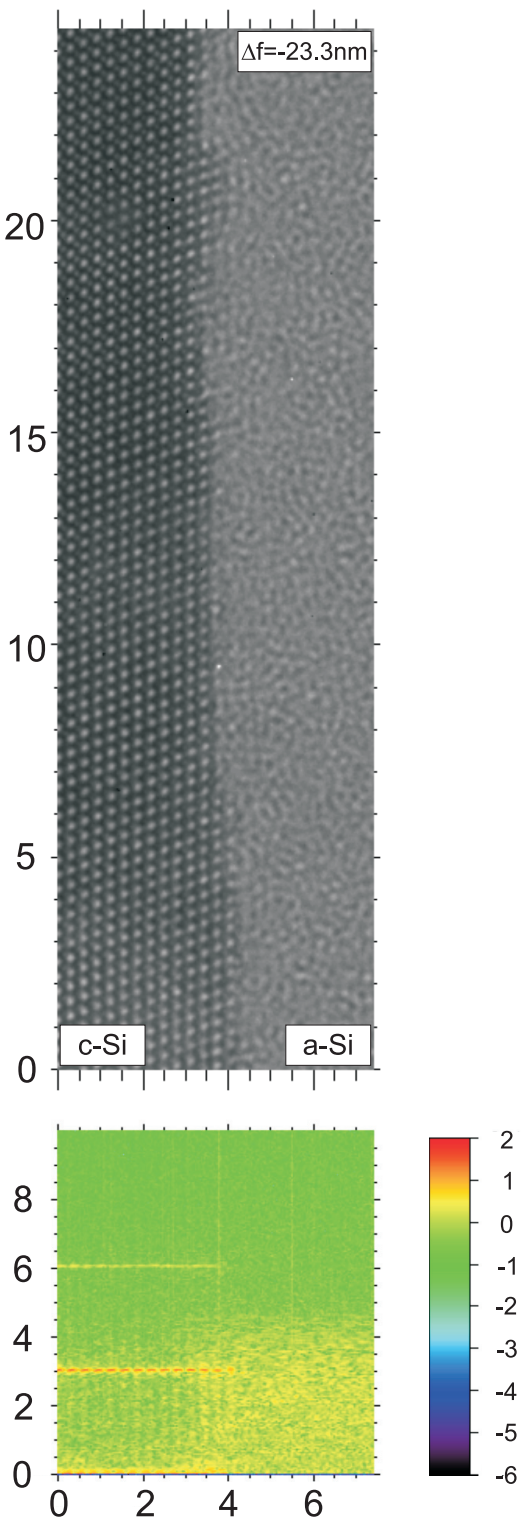

Abbildung 3.1 - Teil einer elektronenmikroskopischen Abbildung der a-Ge/c-Si- (a) bzw. a-Si/c-SiGrenzfläche (b). Die Abbildung entspricht jeweils der Einstellung des minimalen Kontrasts, also einem Defokus von $\Delta f=-21.6 \mathrm{~nm}$ für die a-Ge/c-Si-Probe und $\Delta f=-23.3 \mathrm{~nm}$ für die a-Si/c-Si-Probe. Die Intensitätsverläufe parallel zur Grenzfläche wurden extrahiert und jeweils einer Fourieranalyse zur Ermittlung der vorhandenen Raumfrequenzen unterzogen. Jeweils unter den Abbildungen ist der Verlauf der logarithmierten Amplitudenspektren farbkodiert aufgetragen. Es ist für beide Proben gut zu erkennen, dass beide Spektren im Wesentlichen den gleichen Verlauf und ausgeprägte Maxima bei den Raumfrequenzen $k_{y}=1 / d \approx 3 \mathrm{~nm}^{-1}$ und der doppelt periodischen Komponente $k_{y} \approx 6 \mathrm{~nm}^{-1}$ zeigen. Aus dem Fehlen zusätzlicher Maxima im Spektrum des Intensitätsverlaufs an der Grenzfläche folgt, dass beide Intensitätsverteilungen dieselbe Periodizität besitzen. 
keine wichtigen strukturellen Informationen im Grenzbereich verloren gehen. Mögliche Überstrukturen an der Grenzfläche, deren Periode ein Vielfaches der Periode der Kristallstruktur ist, führen zu veränderter Periodizität in den Intensitätsverläufen der Grenzflächenabbildung. Zu diesem Zweck wurden für beide Proben aus jeweils einer elektronenmikroskopischen Abbildung die Intensitätsverläufe parallel zur Grenzfläche extrahiert und einer Fouriertransformation unterzogen. Die Abfolge der logarithmierten und farbkodierten Amplitudenspektren ist jeweils unterhalb des experimentellen Bildausschnitts in Abbildung 3.1 dargestellt.

Es wird für beide Proben deutlich, dass die Amplitudenspektren lediglich bei den Raumfrequenzen $k_{y}=1 / d \approx 3 \mathrm{~nm}^{-1}$ und der doppelt periodischen Komponente $k_{y} \approx$ $6 \mathrm{~nm}^{-1}$ ausgeprägte Maxima zeigen. Aus dem Fehlen weiterer Maxima im Spektrum der Intensitätsverläufe an der Grenzfläche läßt sich folgern, dass keine Überstrukturen an der Grenzfläche existieren. Signale mit höheren Raumfrequenzen als $k_{y} \geq 9.6 \mathrm{~nm}^{-1}$ treten aufgrund der verwendeten Aperturblende von $k_{\mathrm{Ap}}=4.8 \mathrm{~nm}^{-1}$ nicht auf.

\subsection{Iterativer Bildvergleich gemittelter Grenzflächenabbildungen einer Defokusserie}

Die folgenden Abschnitte beschreiben die in dieser Arbeit verwendete Methode des iterativen Bildvergleichs zwischen gemittelten Grenzflächenabbildungen einer experimentellen Defokusserie sowie deren Simulationen. Dabei befasst sich Abschnitt 3.2.1 mit der numerischen Vorbehandlung der experimentellen Defokusserie, und Abschnitt 3.2.2 zeigt, wie diese gemittelten Abbildung simuliert werden können. Wie die Abbildungen quantitativ miteinander verglichen werden, behandelt schließlich Abschnitt 3.2.3.

\subsubsection{Berechnung gemittelter experimenteller Grenzflächenabbildungen}

Da die Probe während der Aufnahme der Defokusserie driftet, muss diese Probenverschiebung zuerst quantifiziert und korrigiert werden. Dies wird in Abschnitt 3.2.1.1 behandelt. Abschnitt 3.2.1.2 erläutert daraufhin kurz das Verfahren zur Bildmittelung.

\subsubsection{Driftkorrektur}

Bevor die experimentellen Abbildungen einer Defokusserie entlang der Grenzfläche gemittelt werden können, muss gewährleistet sein, dass es sich in jeder Abbildung um denselben Probenbereich handelt. Dies ist normalerweise für einen über alle Abbildungen räumlich festen Bereich nicht gegeben, da die Probe während der Aufnahme einer Defokusserie driftet. Während die Aufnahme einer Abbildung mittels der CCD-Kamera 
schnell geschieht $\left(t_{\text {Acq }} \approx 0.5 \mathrm{~s}\right)$, ist die Zeitspanne, die für das Auslesen der Kamera benötigt wird, im Vergleich dazu groß $(t \approx 8 \mathrm{~s})^{*}$. Die Aufnahme einer Defokusserie von 20 Abbildungen dauert somit circa 3 Minuten. Typische Werte für die Probendrift zwischen zwei Aufnahmen liegen im Schnitt bei $x_{\text {drift }} \approx 2 \AA$. Als Folge dieser Drift kann nicht die gesamte Abbildung der Defokusserie benutzt werden.

Zur Korrektur dieser Probenverschiebung zwischen zwei Abbildungen wurde analog zur Arbeit von PLIKAT ${ }^{[24]}$ das Kreuzkorrelationsverfahren benutzt, welches die Ähnlichkeit zweier Abbildungen untersucht ${ }^{[29,78]}$. Die unterschiedliche Defokussierung für zwei aufeinanderfolgende Abbildungen führt zwar dazu, dass die beiden zu vergleichenden Abbildungen grundsätzlich verschieden sind. Es wurde aber bereits gezeigt, dass die Korrelation zwischen zwei elektronenmikroskopischen Abbildungen dabei im Allgemeinen nicht zerstört wird und sie mittels dieser Methode mit hoher Präzision aneinander ausgerichtet werden können ${ }^{[78,104,105]}$.

Für zwei identische Abbildungen ergibt sich bei der Kreuzkorrelation als maximaler Wert 1. In den hier untersuchten Defokusserien betragen die Werte für die a-Si/cSi-Probe im Mittel 0.62(7) und für die a-Ge/c-Si-Probe 0.71(6). Zur Berechnung des Kreuzkorrelationswertes wurde nur der amorphe Bereich der Abbildung benutzt, da der kristalline Bereich aufgrund seiner Periodizität zu keiner eindeutigen Lösung für den Verschiebungsvektor führt.

Abbildung 3.2 zeigt den auf diesem Wege berechneten Verlauf der Verschiebungsvektoren für zwei in dieser Untersuchung verwendete Defokusserien von 20 Abbildungen der a-Si/c-Si- und a-Ge/c-Si-Probe. Der berechnete Driftverlauf für die a-Ge/c-Si-Probe verifiziert das von PLIKAT ${ }^{[24]}$ bereits für diese Serie bestimmte Ergebnis.

Aus dem Driftverlauf über die Defokusserie ergibt sich ein maximaler Probenbereich, der in allen Abbildungen vorhanden ist. Für die a-Si/c-Si-Probe berechnet sich ein Bereich von $981 \times 959$ Bildpunkten (i.e. $26.23 \times 25.64 \mathrm{~nm}^{2}$ ) und für die a-Ge/c-Si-Probe ein Bereich von $954 \times 928$ Bildpunkten (i.e. $25.62 \times 24.94 \mathrm{~nm}^{2}$ ) bei einer ursprünglichen Bildgröße von $1024 \times 1024$ Bildpunkten (i.e. $27.5 \times 27.5 \mathrm{~nm}^{2}$ ) der CCD-Kamera.

\subsubsection{Mittelung von Abbildungen}

Um aus den Intensitätsverteilungen der experimentellen Abbildungen die kristallperiodisch korrelierten Anteile zu extrahieren, wurde analog zur Vorgehensweise von BoRGARDT ET AL. ${ }^{[10-13]}$ und PLIKAT ${ }^{[24,25]}$ eine periodische Mittelung entlang der Grenzfläche durchgeführt. Abbildung 3.3 illustriert das Vorgehen.

Die elektronenmikroskopische Abbildung wird zunächst in Streifen eingeteilt, deren Höhe $d$ gerade der Periode der Intensitätsverteilung entlang der Grenze entspricht (s. Abb. 3.3(a)). Bei dem verwendeten Substrat handelt es sich um Siliziumscheiben mit einer [111]-Orientierung, dies entspricht einer [111]-Richtung senkrecht zur Grenzfläche.

\footnotetext{
${ }^{*}$ Dies bedeutet, dass die Kamera lediglich $6 \%$ der gesamten Zeit einer Aufnahme belichtet wird.
} 
Abbildung 3.2 - Driftverlauf der beiden in dieser Arbeit verwendeten Defokusserien. Schwarz: a-Si/c-Si-Probe, Rot: a-Ge/c-SiProbe. Die Position der ersten Abbildung in beiden Serien ist $(0,0)$.

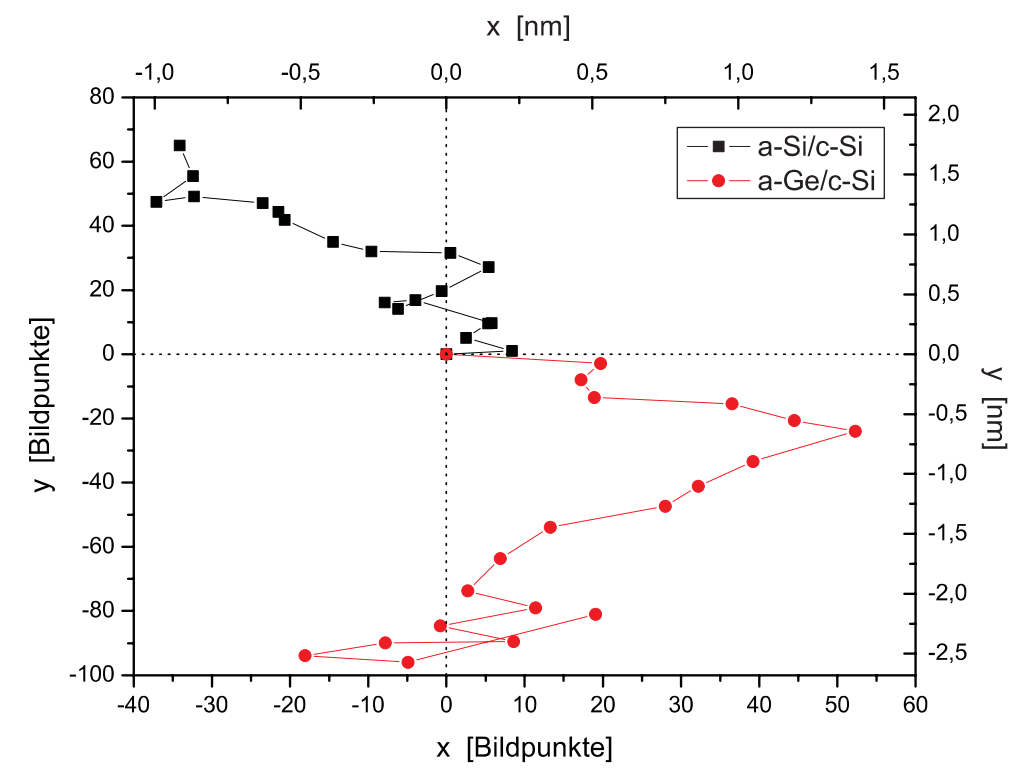

Sie wird im Folgenden als $x$-Achse bezeichnet (s. Abb. 3.4). Für die Richtung entlang der Grenzfläche ergibt sich in der abgebildeten Projektion eine [1112]-Richtung (im Folgenden auch $y$-Achse genannt) und somit eine Streifenhöhe von $d=\sqrt{3 / 8} a_{\mathrm{Si}}=3.326 \AA\left(a_{\mathrm{Si}}=\right.$ $\left.0.5431 \mathrm{~nm}^{[107]}\right)$.

Es wird über äquivalente Punkte in allen Streifen gemittelt:

$$
I^{\prime}(x, y)=\frac{s_{d}(y)}{N} \sum_{n=-(N-1) / 2}^{(N-1) / 2} I(x, y+n d)
$$

Dabei bezeichnet $I^{\prime}(x, y)$ die gemittelte Intensitätsverteilung, $I(x, y)$ die Intensitätsverteilung in der experimentellen Abbildung, $s_{d}$ die Spaltfunktion, für welche

$$
s_{d}(y)=\left\{\begin{array}{lll}
1 & : & -\frac{d}{2} \leq y<\frac{d}{2} \\
0 & : & \text { sonst }
\end{array}\right.
$$

gilt, und $N$ steht für die Anzahl der Streifen.

Dieses Vorgehen ist gleichbedeutend mit einer Reduktion der Information in der Grenzflächenabbildung auf die $d$-periodischen Anteile. Abbildung 3.3(c) zeigt die mittlere Grenzflächenabbildung. Dabei ist zu beachten, dass lediglich der obere Streifen das Ergebnis der Mittelung zeigt, welcher zur besseren Veranschaulichung mehrmals untereinander dargestellt wird. Die mittlere Grenzflächenabbildung enthält die zu erwartenden Merkmale:

- Auf der kristallinen Seite reproduziert sich aufgrund der Periodizität des Kristalls das kristalline Muster. 


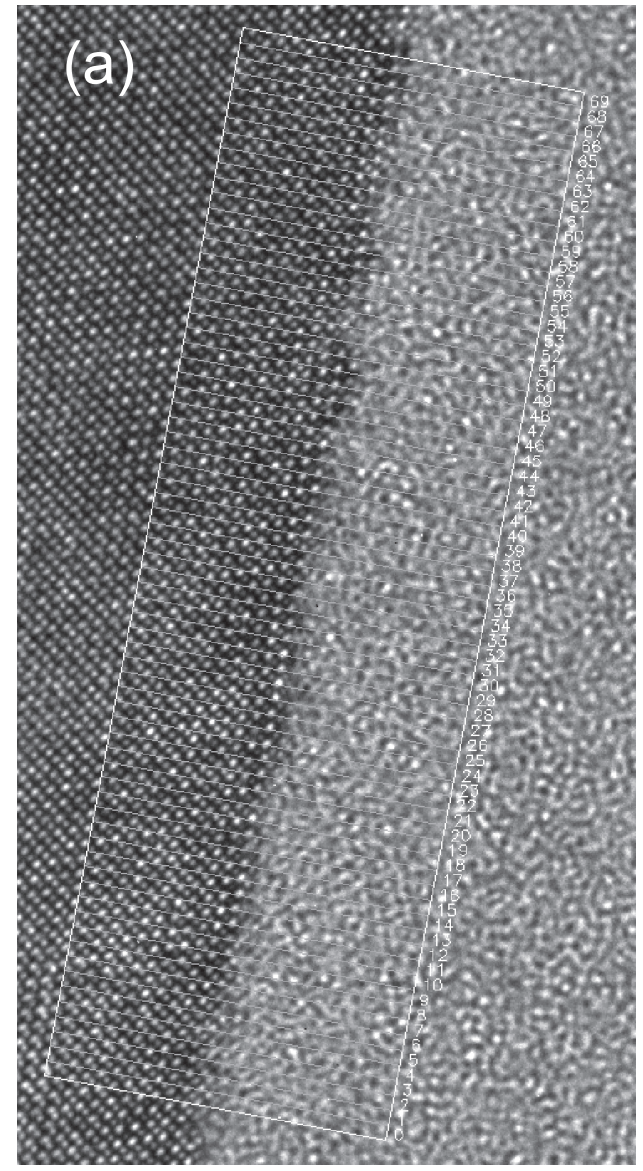

(b)

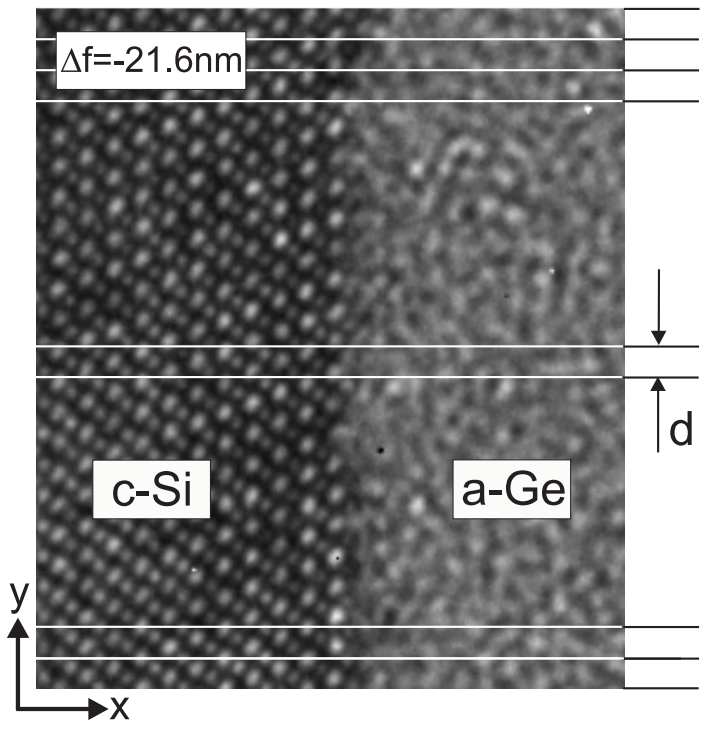

(c)

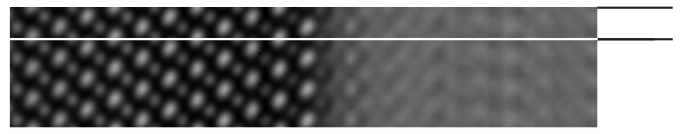

(d)

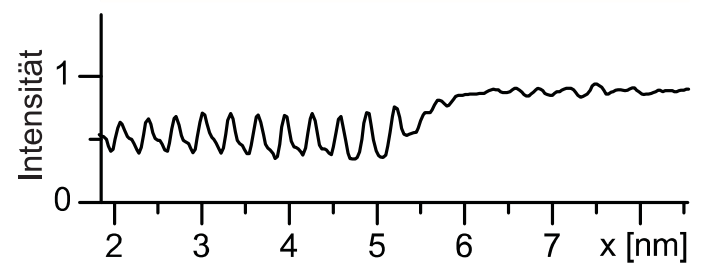

Abbildung 3.3 - Vorgehen bei der Mittelung. (a) Eine Abbildung der Defokusserie der a-Ge/c-SiProbe mit bereits durchgeführter Einteilung in Elementarstreifen der Höhe $d$ senkrecht zur Grenzfläche. (b) Ausschnitt der in Elementarstreifen eingeteilten Grenzflächenabbildung und Mittelung äquivalenter Bildpunkte aller Streifen. (c) Resultierende gemittelte Grenzfl̈̈chenabbildung nach Gleichung 3.1; zur besseren Sichtbarkeit vierfach untereinander dargestellt. (d) Das resultierende Intensitätsprofil nach Gleichung 3.3. Die Abbildung ist der Veröffentlichung von THIEL ET AL. ${ }^{[106]}$ entnommen.

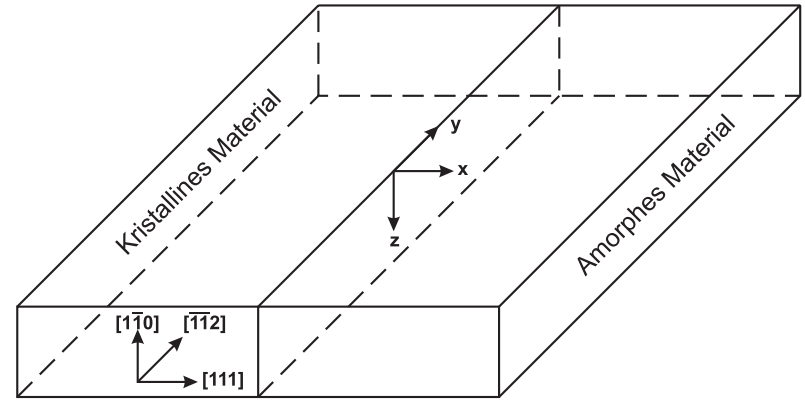

Abbildung 3.4 - Orientierung der Probe. Auf der linken Seite befindet sich das kristalline und auf der rechten Seite das amorphe Material. Die Richtung senkrecht zur Grenzfläche wird als $x$-Achse bezeichnet und ist eine [111]-Richtung. Die $y$-Achse verläuft entlang der Grenzfläche und ist eine [1̄12]-Richtung. Der Elektronenstrahl läuft parallel zur z-Richtung, einer [1110]Richtung. 
- Im amorphen Teil, weit entfernt von der Grenzfläche, ergibt sich eine homogene Intensitätsverteilung, da die Atome - bezogen auf den Kristall - zufällig verteilt sind.

Eine weitere Möglichkeit, die mittleren Grenzflächenabbildungen zu visualisieren, besteht in der Darstellung durch eindimensionale Intensitätsprofile, die wie folgt definiert sind:

$$
\bar{I}(x)=\frac{1}{A} \int_{-A / 2}^{A / 2} I(x, y) d y
$$

Dabei bezeichnet $A=N d$. Abbildung 3.3(d) zeigt ein solches Intensitätsprofil.

Die gemittelten Intensitätprofile sind gut geeignet für einen visuellen Vergleich zwischen den späteren Simulationsergebnissen und dem Experiment. Sie wurden ausschließlich zu diesem Zweck verwendet, während quantitative Vergleiche zwischen Simulation und Experiment auf der Basis der gemittelten zweidimensionalen Grenzflächenabbildungen durchgeführt wurden (s. auch Abschnitt 3.2.3).

\subsubsection{Multislice-Simulation gemittelter Abbildungen}

In diesem Abschnitt wird gezeigt, wie die experimentellen gemittelten Grenzflächenabbildungen simuliert werden können. Dazu wird zunächst in Abschnitt 3.2.2.1 die herkömmliche Simulationsmethode, die so genannte „Multislice“-Simulation beschrieben. Auf welchen Wegen gemittelte Abbildungen mittels der Multislice-Methode simuliert werden können, und die Eigenschaften und Folgerungen, der für diese Arbeit verwendeten „Averaged-Projected-Potential approximation“ (APP-Näherung), sollen in Abschnitt 3.2.2.2 diskutiert werden. Die Konstruktion der zweidimensionalen Verteilungsfunktion, die die für die Simulationen verwendete Beschreibung der mittleren Verteilung des amorphen Materials in Grenzflächennähe ist, wird abschließend in Abschnitt 3.2.2.3 erläutert.

\subsubsection{Der Multislice-Formalismus}

Die Multislice-Methode ${ }^{[14-18]}$ ist ein etabliertes Verfahren zur Simulation elektronenmikroskopischer Abbildungen und z.B. im EMS-Programmpaket von STADELMANN [77] integriert.

Die Modifikation einer einfallenden Elektronenwelle durch das Streupotential einer Probe wird im Rahmen dieser Methode als eine Folge wiederholter Streuung an dünnen Scheiben (,slices") beschrieben. Der Streuprozeß beim Durchlaufen einer einzelnen Scheibe $j$ teilt sich dabei in zwei Teile - Transmission und Propagation - auf. Dieser Ansatz ist möglich, weil die Rückstreuung hochenergetischer Elektronen vernachlässigt werden kann $^{[15]}$. Des Weiteren konnte schon früher nachgewiesen werden ${ }^{[16,17]}$, dass im Limes verschwindender Scheibendicke, $\lim \Delta z \rightarrow 0$, dieser Formalismus in die Beschreibung 
von Elektronenstreuung durch die relativistisch korrigierte Schrödingergleichung unter Vernachlässigung von Rückstreuung übergeht. Abbildung 3.5 illustriert das Vorgehen.

Im Folgenden werden die beiden Schritte - Transmission und Propagation - genauer erläutert:

1. Transmission: Anstelle der Streuung der einfallenden Elektronenwelle am dreidimensionalen Streupotential wird die Streuung lediglich an dem auf die Eintrittsfläche des Elektronenstrahls projizierten Potential $\varphi^{(j)}(x, y)$ berechnet (s. Abb. $3.5(\mathrm{c}))$.

$$
\varphi^{(j)}(x, y)=\frac{1}{\Delta z} \int_{z_{j-1}}^{z_{j}} V(\vec{r}) d z \quad \text { mit } \Delta z=z_{j}-z_{j-1}
$$

$\varphi^{(j)}(x, y)$ bezeichnet dabei das projizierte Potential der $j$-ten Scheibe.

Die Streuung der Elektronenwelle wird durch eine Transmissionsfunktion $P^{(j)}(x, y)$ beschrieben, welche zu einer Phasenschiebung der Elektronenwelle proportional zum projizierten Potential führt. Dabei muss für alle Scheiben die Phasennäherung erfüllt sein, d.h. dass der einfallenden Elektronenwelle beim Durchlaufen der Probenscheibe relativ zu einer Elektronenwelle, die dieselbe Strecke im Vakuum durchläuft, lediglich ein Phasenschub aufgeprägt wird.

$$
\begin{aligned}
P^{(j)}(x, y) & =\exp \left(i \sigma \varphi^{(j)}(x, y)\right) \\
\text { mit } \sigma & =\frac{\pi}{\lambda U_{R}}
\end{aligned}
$$

Dabei bezeichnet $\lambda$ die Wellenlänge der Elektronen, $U_{R}$ die relativistisch korrigierte Beschleunigungsspannung des Mikroskops und $\sigma$ die Wechselwirkungskonstante.

Da in dem in dieser Arbeit betrachteten Fall die Streupotentiale aller Streifen gleich sind, sind ebenso die projizierten Potentiale und Transmissionsfunktionen identisch. Deswegen wird im Folgenden auf den Index $j$ verzichtet.

2. Propagation: Die Ausbreitung der am projizierten Potential der $j$-ten Scheibe gestreuten Elektronenwelle über die Strecke $\Delta z$ durch Vakuum zum projizierten Potential der $j+1$-ten Scheibe wird durch die Faltung der Wellenfunktion mit dem Fresnelpropagator $\mathrm{FP}_{\Delta z}(x, y)$

$$
\mathrm{FP}_{\Delta z}(x, y)=\frac{1}{i \lambda \Delta z} \exp \left[\frac{i \pi}{\lambda \Delta z}\left(x^{2}+y^{2}\right)\right]
$$

ausgedrückt.

Die mathematische Beschreibung eines kompletten Rekursionsschritts für die Modifikation einer einfallenden Elektronenwelle $\psi^{(j)}(x, y)$, die an einer Scheibe mit Transmissions- 


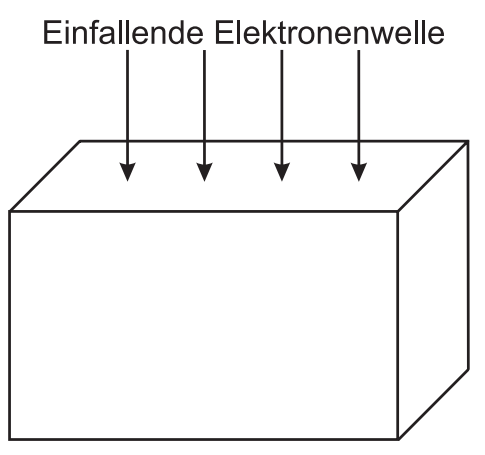

(a) Dicke Probe

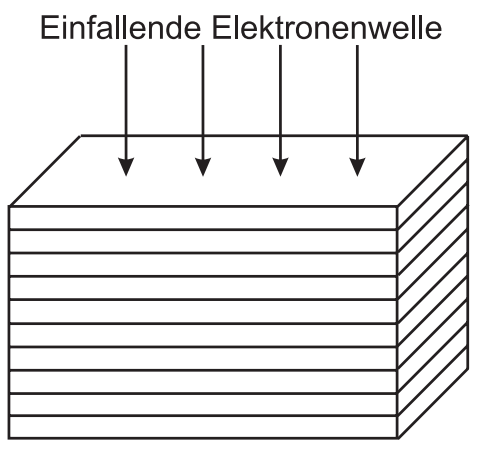

(b) Einteilung in Scheiben

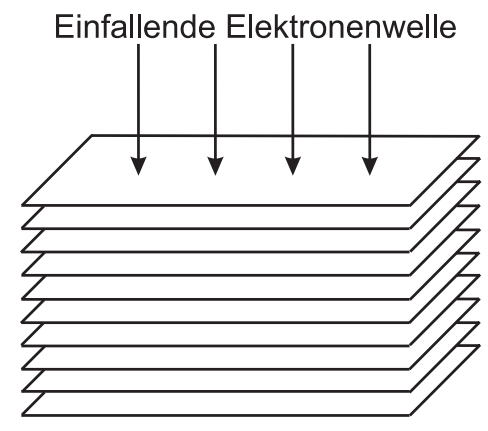

(c) Projektion

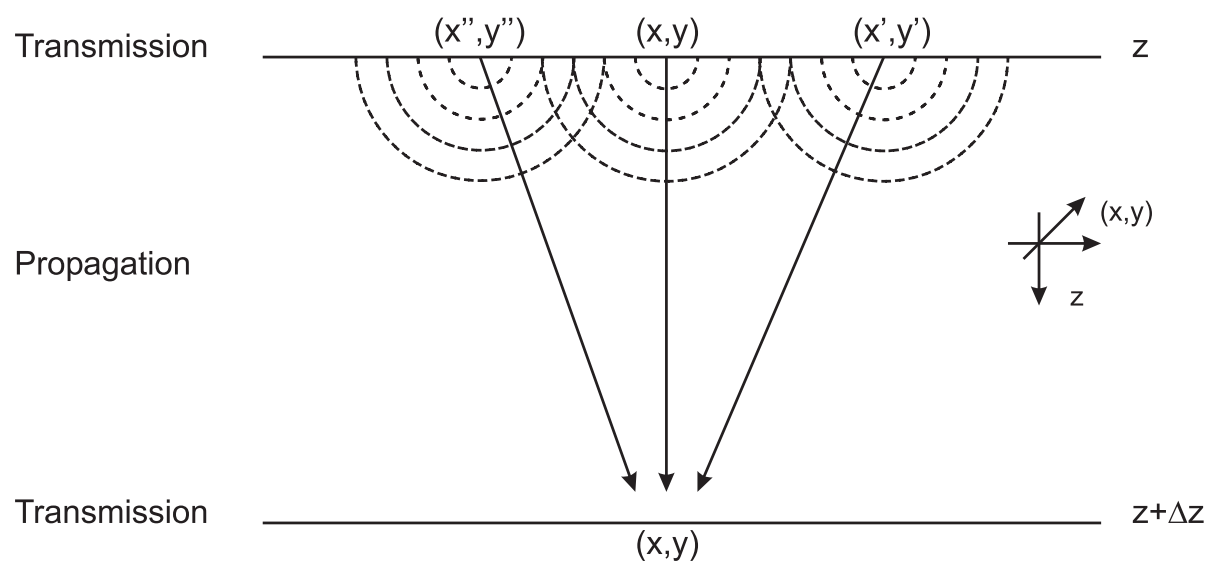

(d) Transmission und Propagation

Abbildung 3.5 - Schematische Darstellung der Multislice-Methode. (a) Die originale, dicke Probe. (b) Einteilung der Probe in dünne Scheiben der Dicke $\Delta z$. (c) Jede Scheibe wird repräsentiert durch die Projektion des Potentials auf eine Ebene. (d) Die gesamte Streuung der Elektronenwelle an der Probe wird somit ausgedrückt durch eine wiederholte Folge von Streuung am projizierten Potential und Propagation der Welle durch Vakuum zwischen den Scheiben. Die Abbildungen sind der Arbeit von KIRKLAND $^{[108]}$ entnommen. 
funktion $P(x, y)$ gestreut wird und sich bis zur nächsten Scheibe im potentialfreien Raum mittels des Fresnelpropagators $\mathrm{FP}_{\Delta z}(x, y)$ über eine Strecke $\Delta z$ ausbreitet, lautet zusammengefasst:

$$
\psi^{(j+1)}(x, y)=\left[P(x, y) \cdot \psi^{(j)}(x, y)\right] \circledast \mathrm{FP}_{\Delta z}(x, y)
$$

\subsubsection{Simulation gemittelter Grenzflächenabbildungen: Die „Averaged-Projected-Potential“'-Näherung}

Die im Abschnitt 3.2.1.2 berechneten mittleren Grenzflächenabbildungen können mittels der eben vorgestellten Multislice-Methode auf zwei Wegen simuliert werden:

Atomistischer Ansatz: Hierbei werden durch die Multislice-Methode elektronenmikroskopische Abbildungen simuliert und anschließend gemittelt. In die Simulationen gehen auf der kristallinen Seite der Grenzfläche die bekannten Atompositionen ein, während sie für die amorphe Seite zunächst nicht bekannt sind und z.B. über „,continuous-random-network“-Modelle oder Molekulardynamiksimulationen berechnet werden müssen. Aufgrund der hohen Anzahl möglicher struktureller Realisierungen ist jedoch die Beschreibung von amorphen Materialien anhand von exakten Positionen individueller Atome nicht sinnvoll. Zudem hat dieses Vorgehen einen weiteren, praktischen Nachteil: Um die Größe der experimentell zugänglichen Mittelungsbereiche zu erreichen, müssten Systemgrößen von $10^{4}-10^{5}$-Atomen gerechnet werden, was lange Simulationszeiten mit sich bringt.

APP-Näherung: Eine gute Alternative stellt die direkte Simulation gemittelter Abbildungen dar. Hierbei werden durch die Multislice-Methode in einem Schritt gemittelte elektronenmikroskopische Abbildungen simuliert. Dieses Verfahren wurde von BORGARDT ET AL. ${ }^{[1]}$ vorgeschlagen und als „Averaged-Projected-Potential“ (APP)-Näherung bezeichnet. Dieses Vorgehen unterscheidet sich von der herkömmlichen Multislice-Methode dadurch, dass statt des projizierten Potentials $\varphi(x, y)$ ein mittleres projiziertes Potential $\varphi^{\mathrm{APP}}(x, y)$ für das amorphe Material benutzt wird. Anstelle der individuellen Atompositionen, welche für den atomistischen Ansatz nötig sind, wird das amorphe Material anhand einer mittleren, zweidimensionalen atomaren Dichtefunktion $\rho(x, y)$ innerhalb eines Streifens mit Ausdehnung $d$ beschrieben ${ }^{\dagger}$. Die Weite $d$ entspricht dabei der Periode der Gitterabbildung entlang der Grenzfläche, wie in Abschnitt 3.2.1.2 bereits erläutert wurde. Dieses Vorgehen reduziert die Simulationszeit erheblich.

Im Folgenden werden die Grundzüge der APP-Näherung genauer erläutert.

Die APP-Näherung beruht, wie gesagt, lediglich auf der mittleren zweidimensionalen Dichte $\rho(x, y)$ der Atome innerhalb eines Streifens mit Ausdehnung $d$. Nach BorGARDT

\footnotetext{
${ }^{\dagger}$ Auch an dieser Stelle wurde auf den Index $j$ für die Scheibe verzichtet.
} 
ET AL. ${ }^{[11]}$ ist:

$$
\begin{aligned}
\varphi^{\mathrm{APP}}(x, y)= & \frac{s_{d}(y)}{\Delta z} \sum_{n=-(N-1) / 2}^{(N-1) / 2} \int_{z_{j-1}}^{z_{j}} \widetilde{V}\left(p_{x}, \frac{n}{d}, 0\right) \widetilde{\rho}\left(p_{x}, \frac{n}{d}\right) \\
& \times \exp \left[2 \pi i\left(p_{x} x+\frac{n}{d} y\right)\right] d p_{x}
\end{aligned}
$$

$\widetilde{\rho}\left(p_{x}, p_{y}\right)$ und $\widetilde{V}\left(p_{x}, p_{y}\right)$ kennzeichnen jeweils die Fouriertransformierten von $\rho(x, y)$ bzw. $V(x, y) \cdot s_{d}(y)$ ist die Spaltfunktion, für die wiederum

$$
s_{d}(y)=\left\{\begin{array}{lll}
1 & : & -\frac{d}{2} \leq y<\frac{d}{2} \\
0 & : & \text { sonst }
\end{array}\right.
$$

gilt (s. Gleichung 3.2).

Die Funktion $\rho(x, y)$ ist die zweidimensionale Projektion der dreidimensionalen atomaren Dichte innerhalb der $j$-ten Scheibe entlang des Elektronenstrahls. Dabei bedeutet $\rho(x, y) d x d y$ die mittlere Anzahl der Atome innerhalb der Fläche $d x d y$ bei $(x, y)$.

BORGARDT ET AL. ${ }^{[11]}$ haben analytisch gezeigt, dass die Beiträge zur gemittelten Bildintensität, welche linear in der Fouriertransformierten des projizierten Potentials (s. Gleichung 3.4) enthalten sind, durch diesen Ansatz korrekt beschrieben werden. Dabei stehen die linearen Beiträge für Elektronen, welche beim Durchlaufen durch die Probe einmal gestreut werden, sich dann durch den Rest der Probe und das Mikroskop ausbreiten und schließlich mit dem ungebeugten Strahl interferieren. Beiträge von zweifach gestreuten Elektronen werden ebenso korrekt beschrieben, solange der Abstand der Scheiben, an denen sie gestreut werden, gegenüber der Nahordnung im amorphen Material groß ist. Ansonsten könnten sich die lokale Atomverteilung und die mittlere Atomverteilung stark voneinander unterscheiden. Abweichungen existieren jedoch für die Beschreibung der Interferenz zweier Elektronen, welche an derselben Scheibe gestreut werden. Anhand von Testrechnungen konnten BORGARDT ET AL. jedoch zeigen, dass dieser Fehler keinen signifikanten Einfluß auf die gemittelten Intensitäten ausübt ${ }^{[11]}$.

Folglich können gemittelte Abbildungen mithilfe der Multislice-Methode hinreichend genau simuliert werden, wenn die Transmissionsfunktion (s. Gleichung 3.5) einer Scheibe für den kristallinen Probenteil auf der Basis des atomistischen Ansatzes also der bekannten Atompositionen und für den amorphen Probeteil durch das zweidimensionale mittlere projizierte Potential aufgestellt wird.

\subsubsection{Konstruktion einer zweidimensionalen Verteilungsfunktion}

Die Konstruktion der in die Simulationen eingehenden zweidimensionalen Verteilungsfunktion $\rho(x, y)$ für das amorphe Material soll im Folgenden erläutert werden. Die auf den nächsten Seiten dargestellte Vorgehensweise ist der Veröffentlichung von BoRGARDT 
ET AL. ${ }^{[13]}$ entnommen, wird aber an dieser Stelle aufgrund ihrer Wichtigkeit für das Verständnis der Beschreibung der mittleren Verteilung des amorphen Materials in Grenzflächennähe nochmals skizziert. Am Ende dieses Abschnitts soll dann die für diese Arbeit durchgeführte Änderung des ursprünglichen Ansatzes von BORGARDT ET AL. ${ }^{[13]}$ beschrieben und diskutiert werden.

Ausgangspunkt für die Konstruktion der dreidimensionalen Verteilungsfunktion $\rho_{3 \mathrm{D}}$ ist die Näherung eines starren Substrats, d.h. die Annahme, dass die Positionen der letzten kristallinen Atome nicht durch das amorphe Material beeinflusst werden. Diese Näherung wird üblicherweise auch für die Struktur des festen Materials bei der elektronenmikroskopischen Untersuchung von grenzflächeninduzierter Ordnung an fest/flüssigen Grenzflächen gemacht ${ }^{[21]}$.

Ausgehend von dieser Einschränkung ist die Konstruktionsvorschrift von BorGARDT ET AL. ${ }^{[13]}$ ein rekursives Vorgehen, bei dem die Kristallstruktur des Substrats Atomlage für Atomlage in das amorphe Material fortgesetzt wird. Für die Bindungslänge zwischen den Atomen des amorphen Materials und die Winkelverteilung dieser Bindungen relativ zu den mittleren Orientierungen werden aber die Gaussverteilungen $W_{r}(r)$ und $W_{\theta i}\left(\Delta \theta_{i}\right)$ verwendet, so dass durch das rekursive Vorgehen die kristalline Ordnung mit zunehmender Entfernung von der Grenzfläche abnimmt bzw. $\rho(x, y)$ in eine homogene Verteilung übergeht.

Es folgt eine kurze Beschreibung der beiden Gaussfunktionen:

Verteilung der Bindungslängen $W_{r}(r)$ steht für die Verteilung der Bindungslängen. Für sie wird angenommen, dass sie sich um den mittleren Wert für das reine amorphe Material im Volumen, $r_{0}$, entsprechend der Gaussfunktion

$$
W_{r}(r)=\frac{1}{\sqrt{2 \pi} \sigma_{r}} \exp \left(-\frac{1}{2} \frac{\left(r-r_{0}\right)^{2}}{\sigma_{r}^{2}}\right)
$$

verteilen. Die Werte für $r_{0}$ und $\sigma_{r}$ werden dabei als konstant für das gesamte amorphe Material angenommen.

Für das amorphe Germanium wurde als mittlerer Wert für die Bindungslänge $r_{0}=0.2463 \mathrm{~nm}$ und eine Standardabweichung von $\sigma_{r}=0.0074 \mathrm{~nm}$ entsprechend den Werten von EtheringtoN ${ }^{[26]}$ benutzt. Der Wert für die erste Bindungslänge zwischen dem Siliziumsubstrat und dem Germanium wurde als das arithmetische Mittel aus $r_{0}$ und der Bindungslänge des Siliziums $\left(r_{\mathrm{Si}}=0.2355 \mathrm{~nm}^{[109]}\right)$ festgelegt und beträgt somit $r_{1}=0.2408 \mathrm{~nm}$. Die Werte für das amorphe Silizium mit $r_{0}=$ $0.235 \mathrm{~nm}$ und $\sigma_{r}=0.0065 \mathrm{~nm}$ entstammen der Arbeit von LAAZIRI ${ }^{[109]}$.

Verteilung der Abweichungen von den mittleren Bindungsrichtungen Analog da$\mathrm{zu}$ beschreibt die Funktion $W_{\theta i}\left(\Delta \theta_{i}\right)$ die Verteilung der Abweichungen $\Delta \theta_{i}$ der Bindungsrichtungen zu den mittleren räumlichen Orientierungen der Bindungen 
Abbildung 3.6 - Graphische Illustration der Konstruktion der mittleren atomaren Dichte $W_{i}^{(1)}\left(\vec{r}, \vec{r}_{k}\right)$ in der Atomlage $i$ bei einer Rückbindung ausgehend von einem kristallinen Atom am Ort $\vec{r}_{k}$ in der Atomlage $(i-1)$.

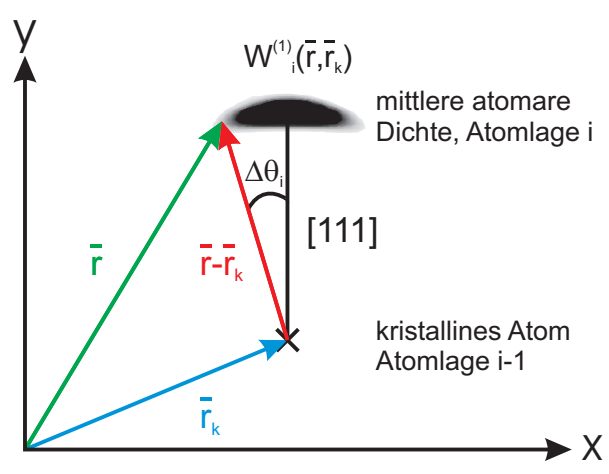

anhand der Gaussfunktion:

$$
W_{\theta i}\left(\Delta \theta_{i}\right)=\frac{1}{\sqrt{2 \pi} \sigma_{\theta i}} \exp \left(-\frac{1}{2} \frac{\Delta \theta_{i}^{2}}{\sigma_{\theta i}^{2}}\right)
$$

$\sigma_{\theta i}$ ist die Standardabweichung in der $i$-ten atomaren Lage. Die mittleren Orientierungen der Bindungen ergeben sich aus dem der Konstruktion zugrunde liegenden Gitter.

Als erstes soll die Situation betrachtet werden, bei der die mittlere Orientierung der Bindungen ähnlich der Normalen der Grenzfläche ist. In diesem Fall sind die Atome in der $i$-ten atomaren Lage mit lediglich einer Rückbindung an die $(i-1)$-Lage gebunden. Abbildung 3.6 illustriert die aus den beiden Funktionen 3.11 und 3.12 resultierende mittlere atomare Dichte in der $i$-ten Atomlage, ausgehend von einem kristallinen Atom an der Position $\vec{r}_{k}$ in der $(i-1)$-ten Atomlage. Sie wird durch

$$
W_{i}^{(1)}\left(\vec{r}, \vec{r}_{k}\right) \equiv W_{i}^{(1)}\left(\vec{r}-\vec{r}_{k}\right)=C W_{r}\left(\left|\vec{r}-\vec{r}_{k}\right|\right) W_{\theta i}\left(\Delta \theta_{i}\right)
$$

berechnet. $\Delta \theta_{i}$ beschreibt den Winkel zwischen $\left(\vec{r}-\vec{r}_{k}\right)$ und der entsprechenden mittleren Orientierung der Bindung entlang der [111]-Richtung. $C$ bezeichnet die Konstante, welche das Integral

$$
\int W_{i}^{(1)}\left(\vec{r}, \vec{r}_{k}\right) d \vec{r}=1
$$

normiert.

Diese Beschreibung eignet sich aber nur für die erste „amorphe“ Atomlage, welche an die letzte dem Kristall zugeordnete Atomlage angeheftet ist. Im allgemeinen Fall ist der Ausgangspunkt bereits eine Verteilung der atomaren Dichte. Hierfür wurde angenommen, dass die Funktion $W_{i}^{(1)}\left(\vec{r}, \vec{r}^{\prime}\right)$ für alle Positionen $\overrightarrow{r^{\prime}}$ der Verteilung der atomaren Dichte des $k$-ten Atoms in der Nähe zu dessen Schwerpunkt $\vec{r}_{k}$ gleich bleibt. Dann ergibt 
sich für die mittlere atomare Dichte $f_{i j}(\vec{r})$ des $j$-ten Atoms in der $i$-ten Lage:

$$
f_{i j}(\vec{r})=\int f_{(i-1) k}\left(\overrightarrow{r^{\prime}}\right) W_{i}^{(1)}\left(\vec{r}-\overrightarrow{r^{\prime}}\right) d \overrightarrow{r^{\prime}}=f_{(i-1) k}(\vec{r}) \circledast W_{i}^{(1)}(\vec{r})
$$

Dabei steht $f_{(i-1) k}\left(\vec{r}^{\prime}\right)$ für die mittlere atomare Dichte am Punkt $\vec{r}_{k}$.

Für die zweite Möglichkeit, dass das $j$-te Atom drei Rückbindungen zur (i-1)-ten Lage aufweist, wurde als mittlere atomare Dichte $f_{i j}(\vec{r})$ das Mittel der drei entsprechenden Verteilungen angenommen.

$$
\begin{aligned}
f_{i j}(\vec{r}) & =\frac{1}{3} \sum_{k=1}^{3} \int f_{(i-1) k}\left(\overrightarrow{r^{\prime}}\right) W_{i j k}^{(3)}\left(\vec{r}-\overrightarrow{r^{\prime}}\right) d \overrightarrow{r^{\prime}} \\
& =\frac{1}{3} \sum_{k=1}^{3} f_{(i-1) k}(\vec{r}) \circledast W_{i j k}^{(3)}(\vec{r})
\end{aligned}
$$

Dabei unterscheidet sich die Funktion $W_{i j k}^{(3)}\left(\vec{r}-\overrightarrow{r^{\prime}}\right)$ von der oben eingeführten Funktion $W_{i}^{(1)}\left(\vec{r}-\overrightarrow{r^{\prime}}\right)$ durch die beiden weiteren Indizes $j$ und $k$, die die drei möglichen mittleren Orientierungen der Bindungen bezeichnen, zu denen die Abweichungen $\Delta \theta_{i}$ gemessen werden.

In dieser allgemeinen Darstellung wird die letzte dem Kristall zugeordnete Atomlage durch die Funktion

$$
f_{0 j}(\vec{r})=\delta\left(\vec{r}-\vec{r}_{b j}\right)
$$

ausgedrückt.

Die dreidimensionale atomare Dichte für die amorphe Schicht ergibt sich letztlich zu

$$
\rho_{3 \mathrm{D}}(\vec{r})=\sum_{i} \sum_{j} f_{i j}(\vec{r})
$$

und die zweidimensionale Projektion innerhalb einer Superzelle der Höhe $c$ zu:

$$
\rho(x, y)=\int_{0}^{c} \rho_{3 \mathrm{D}}(\vec{r}) d z
$$

Abbildung 3.7a-c illustriert die Konstruktion für die ersten beiden atomaren Lagen graphisch, und 3.7d zeigt schließlich $\rho(x, y)$ für mehrere Lagen. Es ist klar zu erkennen, dass die an das Modell gestellte Forderung, die in das amorphe Material induzierten, kristallperiodischen Korrelationen sollen mit zunehmender Entfernung von der Grenzfläche abnehmen, erfüllt ist. Dies wird durch die Vorgehensweise bei der Konstruktion 
Atomare Dichte $\left[\mathrm{nm}^{-2}\right]$

(a)

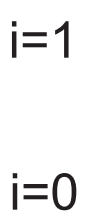

(b)

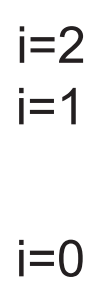

$\mathrm{Ge}$

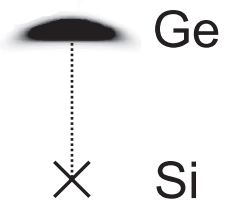

$\mathrm{Si}$ (d)



$>75.00$

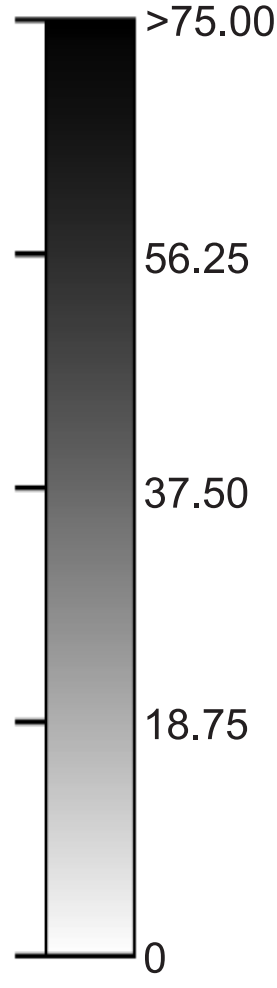

(c)

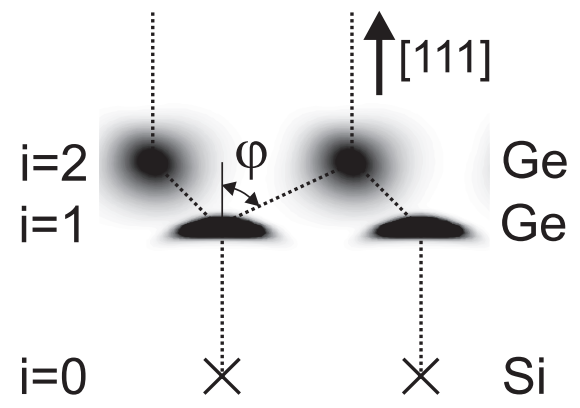

$\mathrm{Ge}$ $\mathrm{Ge}$

$\mathrm{Si}$ Ge

$\mathrm{Si}$

Abbildung 3.7 - Schema zur Konstruktion der dreidimensionalen Verteilungsfunktion $\rho_{3 D}(x, y)(a, b)$ sowie deren zweidimensionaler Projektion $\rho(x, y)(c, d)$. (a) Ausgangspunkt ist die letzte Lage des kristallinen Substrats $(i=0)$. Ausgehend von ihr wird die mittlere Orientierung der Bindungen festgelegt (gepunktete Linie). Es werden nun Variationen für die Bindungslänge (vorher festgelegt und für alle atomaren Lagen gleich) und für die Abweichung von der mittleren Orientierung der Bindungen abhängig von der Lage $i$ zugelassen. Diese Variationen werden durch die Gaussfunktionen $W_{r}(r)$ und $W_{\theta i}\left(\Delta \theta_{i}\right)$ beschrieben. Das Ergebnis ist eine kegelförmige Verteilung. (b) Rekursive Fortsetzung durch die Gaussfunktionen für die zweite atomare Lage, deren mittlere Orientierung der Bindungen um einen Winkel $\varphi$ geneigt ist. Die Auswirkungen der Gaussfunktionen sind wiederum durch die Kegel angedeutet. Die beiden durchgezogenen Linien zeigen zwei mögliche Bindungsorientierungen in Projektion entlang der [11̄0]-Richtung zwischen dem Silizium und dem Germanium. (c) Resultierende zweidimensionale Projektion von $\rho_{3 D}(x, y)$ für die ersten zwei Lagen. (d) Zweidimensionale Verteilungsfunktion berechnet für mehrere Lagen. Gut zu erkennen ist die zunehmende Homogenität der Verteilung mit wachsendem Abstand zur Grenzfläche. Abbildungen a-c aus der Veröffentlichung von BoRGARDT ET AL. [13] entnommen. 
von $\rho(x, y)$ sichergestellt. Aufgrund der Rekursivität und Faltung verbreitert sich die mittlere atomare Dichte ausgehend von der Deltafunktion der letzten kristallinen Siliziumlage hin zu einer homogenen Verteilung.

Da im Verhältnis zu den weiteren Atomlagen in der ersten Atomlage aufgrund der noch starken Lokalisierung der Atome große Werte in $\rho(x, y)$ erreicht werden, ist eine Darstellung der Verteilungsfunktion mit der gesamten Dynamik anhand einer linearen Graustufenskala nicht sinnvoll. Um dennoch die Variationen jenseits dieser Atomlage sichtbar zu machen, wird allen Werten von $\rho(x, y)$, die größer oder gleich dem heuristischen Wert von $75 \frac{1}{\mathrm{~nm}^{2}}$ sind, analog zu den Veröffentlichungen von BORGARDT ET AL. ${ }^{[11-13]}$ derselbe Farbwert (schwarz) zugeordnet (s. auch Abb. 3.7(d)). Dieses Vorgehen wurde für alle Verteilungsfunktionen in dieser Arbeit verwendet.

Das Ausmaß des Einflusses der Translationssymmetrie des Kristalls auf die Orientierung der Bindungen wird durch die $\sigma_{\theta i}$ beschrieben. Während die physikalische Bedeutung von $\sigma_{\theta 1}$ sofort als die Verteilung der mittleren Orientierung der Bindungen zwischen den Atomen der letzten kristallinen und der ersten amorphen Atomlage an der Grenzfläche interpretiert werden kann, ist dies für die weiteren $\sigma_{\theta i}$ aufgrund der Konstruktion nicht möglich.

Da der orientierende Einfluss des Kristalls auf die Atompositionen im Amorphen mit zunehmendem Abstand zur Grenzfläche abnimmt, müssen sich die Verteilungen der Bindungsrichtungen um die mittlere Bindungsorientierung ständig verbreitern. Deswegen nahmen BorgARDT ET AL. ${ }^{[13]}$ eine lineare Abhängigkeit der $\sigma_{\theta i}$ von der Atomlage $i$ an:

$$
\sigma_{\theta i}=\sigma_{\theta 1}+\alpha(i-1)
$$

Im Gegensatz dazu wurde in dieser Arbeit ein differenzierterer Ansatz gewählt, bei dem die Atomlagen, die nur eine Rückbindung entlang der [111]-Richtung haben, von solchen unterschieden werden, die dazu schräg verlaufen und drei Rückbindungen aufweisen.

$$
\sigma_{\theta i}= \begin{cases}\sigma_{\theta i} & : i=0, \ldots, N \\ \sigma_{\theta N-1}+\alpha_{1}(i-N+1) & : i>N \text { und } i \text { ungerade } \\ \sigma_{\theta N}+\alpha_{2}(i-N) & : i>N \text { und } i \text { gerade }\end{cases}
$$

mit $k=1,2, \ldots$

Bis zur atomaren Lage $N$ ergeben sich somit die $\sigma_{\theta i}$ als unabhängige Parameter. Oberhalb von $N$ werden entsprechend der Anzahl der Rückbindungen die $\sigma_{\theta i}$ linear durch das letzte unabhängige $\sigma_{\theta i}$ und eine Steigung $\alpha$ bestimmt. Durch die größere Anzahl von Parametern wird die Variation in den Bindungwinkeln der einzelnen Atomlagen besser und unabhängig voneinander beschrieben. Die mindestens nötige Anzahl der zu benutzenden Parameter hängt aber von der Ausdehnung des Übergangsbereiches ab und muss somit für jedes System neu definiert werden. Dabei spielt natürlich auch der Rechenaufwand eine Rolle. Je mehr Parameter benutzt werden, desto aufwändiger und 
zeitintensiver sind die numerischen Anpassungen.

Es muss an dieser Stelle jedoch noch einmal betont werden, dass es mithilfe dieses Ansatzes nicht möglich ist, eine konkrete Realisierung der Atompositionen zu bestimmen. Er beschreibt lediglich eine mittlere atomare Verteilung bezogen auf das kristalline Substrat, welche mit der tetraedrisch gebundenen Struktur in Einklang steht.

\subsubsection{Vergleich simulierter und experimenteller Defokusserien}

In Kapitel 2 wurde bereits angesprochen, dass experimentelle und simulierte elektronenmikroskopische Abbildungen aufgrund großer Diskrepanzen in den Kontrasten und mittleren Intensitäten nicht problemlos quantitativ miteinander verglichen werden können. Um dies dennoch zu ermöglichen, wird üblicherweise versucht, diesen Umstand durch Normierung der experimentellen und simulierten Abbildungen zu umgehen ${ }^{[8]}$.

In der vorliegenden Arbeit wurde das unangepasste Bildvergleichsverfahren nach MöBUS benutzt ${ }^{[8]}$. Dabei wird die experimentelle Abbildung auf die mittlere Gesamtintensität im Elektronenstrahl normiert, da Intensitätswerte von simulierten Abbildungen durch die Simulationsprogramme immer relativ zu einer mittleren Intensität im Elektronenstrahl von 1 berechnet werden. Zu diesem Zweck wurde in der vorliegenden Untersuchung neben den eigentlichen Grenzflächenabbildungen am Ende der Defokusserie eine Referenzaufnahme des Elektronenstrahls ohne Probe gemacht. Die auf diese Weise normierte Intensität einer experimentellen Abbildung ergibt sich somit zu:

$$
I_{\text {norm }}(x, y)=\frac{I(x, y)}{<I_{\text {Referenz }}>}
$$

Das Vorgehen zur Normierung der Kontraste mittlerer Grenzflächenabbildungen für die a-Ge/c-Si-Probe wurde bei der in der Arbeit von PLIKAT ${ }^{[24]}$ vorgestellten Methode belassen, da keine Referenzaufnahmen existieren. In diesem Fall wurden die mittleren Intensitäten und die als Standardabweichungen der Intensitätswerte definierten Kontraste im kristallinen Bereich aufeinander normiert.

Um die Unterschiede in den normierten Abbildungen zu quantifizieren, wurde ein Differenzmaß angewendet:

$$
R=\frac{1}{N} \sum_{k} R_{k}\left(I_{\exp }^{(k)}, I_{\text {sim }}^{(k)}\right)=\frac{1}{N} \sum_{k} \sum_{x y} \frac{\left[\left(I_{\text {exp }}^{(k)}\right)_{x y}-\left(I_{\text {sim }}^{(k)}\right)_{x y}\right]^{2}}{\left[\left(I_{\exp }^{(k)}\right)_{x y}\right]^{2}}
$$

Dabei bezeichnet $\left(I_{\exp }^{(k)}\right)_{x y}$ bzw. $\left(I_{\text {sim }}^{(k)}\right)_{x y}$ jeweils den Intensitätswert in der $k$-ten experimentellen bzw. simulierten Abbildung an der Stelle $(x, y)$. Die Gewichtung durch die 
experimentellen Intensitätwerte ist nur deswegen sinnvoll möglich, weil das Rauschen in den Abbildungen nach der Mittelung stark unterdrückt ist. Dieses gewählte Bildvergleichsmaß ist dem aus der Analyse von Röntgenbeugungsmustern bekannten R-Faktor ${ }^{[8]}$ ähnlich.

Computerexperimente haben gezeigt, dass ein solches Maß für die Anpassung der Intensitäten in der Grenzflächenregion geeignet ist ${ }^{[13]}$, da es nicht nur sensitiv auf die Positionen von Maxima und Minima reagiert, sondern zusätzlich Veränderungen in der mittleren Intensität im Übergangsbereich zwischen kristallinem und amorphem Material bewertet.

Schließlich wurde der quantitative Bildvergleich simultan an 20 Abbildungen einer Defokusserie durchgeführt und nicht, wie in der Literatur üblich, lediglich an einer einzelnen experimentellen Abbildung. Wie bereits in Abschnitt 2.3.4 erläutert, führt dieses Vorgehen zu einer Erhöhung der Konfidenz in die angepassten Parameter des Strukturmodells und zu einer zuverlässigen Trennung des Einflusses der Delokalisierung von dem der grenzflächeninduzierten Ordnung bei der Strukturbestimmung.

\subsubsection{Der Einfluss des Aufzeichnungsmediums}

Für die Unterschiede zwischen simulierten und experimentellen Kontrasten spielt auch das Aufzeichnungsmedium eine Rolle. Die Ursache ist die Punktverwaschungsfunktion von CCD-Kameras, welche auch bei kleinen Raumfrequenzen zu einem Verlust von ca. $33 \%$ des Kontrasts führt ${ }^{[91]}$.

Um den Effekt der Punktverwaschung in die simulierten Abbildungen einfließen zu lassen, werden diese nach der Simulation mit der Punktverwaschungsfunktion gefaltet bzw. im Fourierraum mit der Modulationstransferfunktion (MTF) multipliziert.

Die MTF ist die Punktverwaschungsfunktion im Fourierraum ${ }^{[10]}$

$$
\operatorname{MTF}\left(q_{x}, q_{y}\right)=\iint \mathcal{P}(x, y) \exp \left\{2 \pi i\left(x q_{x}, y q_{y}\right)\right\} d x d y
$$

$q_{x}$ und $q_{y}$ stehen für die Komponenten der Raumfrequenz.

Die MTF kann mittels verschiedener Methoden gemessen werden ${ }^{[110-113]}$, die im Detail an dieser Stelle aber nicht diskutiert werden müssen. Für das hier verwendete Mikroskop wurde die MTF von SEIBT ${ }^{[113]}$ bestimmt und durch eigene Messungen bestätigt.

Dabei wurde die MTF durch die Summe aus zwei Lorentz-Linien und einer Konstanten parametrisiert, da dies zu einer besseren Anpassung (kleineres Fehlerquadrat bei gleicher Parameteranzahl) führte als z.B. die Summe zweier Gaussfunktionen ${ }^{[111]}$, d.h.:

$$
\operatorname{MTF}(q)=\frac{a_{1}}{1+\beta_{1} q^{2}}+\frac{a_{2}}{1+\beta_{2} q^{2}}+c
$$

Hierbei stellen $q$ die Raumfrequenz und $a_{1}, \beta_{1}, a_{2}$ und $\beta_{2}$ die Parameter der MTF dar, 
für die die folgenden Werte verwendet wurden: $a_{1}=0.693, \beta_{1}=19.504, a_{2}=0.216$, $\beta_{2}=9227.5$ und $c=0.091$.

Das für diese Untersuchung verwendete Mikroskop ist mit einer „,slow-scan“ (SSC) CCD-Kamera der Firma Gatan (Modell 694) ausgerüstet. Eine genaue Beschreibung der Funktionsweise findet sich bei KRIVANEK UND MOONEY ${ }^{[114]}$.

\subsubsection{Bestimmung der Defoki und der Probendicke}

Für die Simulation der gemittelten Grenzflächenabbildung und somit auch für den Vergleich mit experimentellen Abbildungen sind Probendicke und Defokus wichtige Parameter. Variationen in der Probendicke führen zu einer veränderten Austrittswellenfunktion der Elektronen, und die Defokussierung bestimmt das Übertragungsverhalten des Mikroskops. Während die Kristallstruktur des Siliziumsubstrats bekannt ist und für das amorphe Material eine zweidimensionale Verteilungsfunktion $\rho(x, y)$ benutzt wird, sind die Probendicke des abgebildeten Probenbereiches und die Defokussierung zunächst unbekannt.

Beide Parameter können aber wiederum durch den iterativen Bildvergleich einer experimentellen Defokusserie von Gitterabbildungen mit Simulationen sehr genau bestimmt werden $^{[8]}$. Als Differenzmaß dient dabei ebenfalls der in Gleichung 3.23 definierte RFaktor. Da dieser Weg unter Umständen sehr aufwändig ist, wurde hier zunächst der Konfigurationsraum für den Defokus mittels des in Anhang A beschriebenen Verfahrens der Auswertung von Diffraktogrammen dünner amorpher Proben eingeschränkt und erst danach der iterative Bildvergleich durchgeführt.

Die Defokusschrittweite zwischen den Abbildungen wurde als konstant angenommen, so dass die Beschreibung der Defoki durch den Defokuswert der ersten Abbildung, den Startdefokus $\Delta f_{1}$, und die Defokusschrittweite $\delta \Delta f$ erfolgt.

\subsection{Untersuchung der strukturellen Homogenität entlang der Grenzfläche}

In Abschnitt 3.2 wurde ausführlich besprochen, wie der strukturelle Übergang zwischen kristallinen und amorphen Materialien innerhalb der APP-Näherung mithilfe der zweidimensionalen Verteilungsfunktion $\rho(x, y)$ beschrieben werden kann. Für die Untersuchung der strukturellen Homogenität entlang einer Grenzfläche aber musste diese Methode für die vorliegende Arbeit erweitert werden, was im Folgenden diskutiert werden soll.

Da die Untersuchung an verschiedenen Abschnitten der Grenzfläche durchgeführt werden soll, muss zunächst die Frage nach der minimalen lateralen Ausdehnung des Mittelungsgebietes betrachtet werden. Im Rahmen der in dieser Arbeit verwendeten statistischen Beschreibung des Übergangs sollte dieser Bereich dabei so groß wie möglich gewählt werden. Dies sorgt für eine hohe Signifikanz in $\rho(x, y)$. Im Umkehrschluss führt 
eine Verkleinerung des Mittelungsgebietes zwar zu einer Verbesserung der lateralen Auflösung, jedoch vergrößert sich der Fehler. In Abschnitt 3.3.1 wird nun gezeigt, wie aus der Theorie der APP-Näherung eine robuste Annahme für die untere Grenze der Ausdehnung des Mittelungsbereiches abgeleitet werden kann.

Um die Signifikanz möglicher Unterschiede in zwei Verteilungsfunktionen, die der Anpassung zweier unterschiedlicher Bereiche der Grenzfläche entstammen, abschätzen zu können, muss der Fehler in $\rho(x, y)$ bekannt sein. Die verschiedenen Quellen, die zu diesem Fehler beitragen, sowie dessen Auswirkung auf die zweidimensionale Verteilungsfunktion und dessen Bestimmung werden ausführlich in Abschnitt 3.3.2 behandelt.

\subsubsection{Die laterale Ausdehnung des Mittelungsbereiches}

Innerhalb der in Abschnitt 3.2.2.2 eingeführten APP-Näherung existiert bei der Berechnung der linearen Beiträge zur simulierten gemittelten Abbildung eine Näherung für die Anteile, welche von den Atomen des amorphen Materials am Rand des Mittelungsbereiches stammen. Dabei sind die Beiträge der Atome betroffen, welche sich $\pm d / 2$ oberund unterhalb des Mittelungsbereiches befinden. Ihr Beitrag wird von BoRGARDT ET AL. ${ }^{[11]}$ unter der Vorraussetzung, dass die Anzahl der Streifen $N \gg 1$ ist, vernachlässigt. Somit kann für die gemittelte experimentelle Grenzflächenabbildung gefolgert werden, dass sie sich bei einer Verschiebung des Mittelungsbereiches um einen Streifen nach oben und unten nicht wesentlich ändert. Andernfalls sind die Beiträge am Rand zu groß und das Mittelungsgebiet muss weiter vergrößert werden. Die experimentellen Abbildungen wurden dementsprechend anhand des folgenden und in Abbildung 3.8 graphisch dargestellten Schemas analysiert:

1. Für eine vorgegebene Größe $A=N d$ des Mittelungsgebietes wird die gemittelte Grenzflächenabbildung an einer bestimmten Stelle der Grenzfläche bestimmt. In Abbildung 3.8 ist exemplarisch $N=30$ gewählt.

2. Das Mittelungsgebiet wird nun um einen Streifen (Strecke $d$ ) nach oben verschoben, und wiederum die gemittelte Grenzflächenabbildung berechnet. In Abbildung 3.8 entspricht dies zum einen dem schwarzen Gebiet mit den Streifen 20 bis 49 (ursprünglicher Bereich) und zum anderen dem blauen Gebiet mit den Streifen 21 bis 50 (verschobener Bereich).

3. Für jeden Bildpunkt der beiden gemittelten Grenzflächenabbildungen wird entsprechend eines noch zu bestimmenden Maßes (s. Gleichung 3.26 weiter unten im Text) die Intensitätsvariation in äquivalenten Bildpunkten bestimmt. Der größte Wert für alle Bildpunkte wird erfasst.

4. Die Schritte 1-3 müssen für alle Abbildungen der Defokusserie durchgeführt werden. Der größte Wert der Intensitätsvariation aller Abbildungen gilt als repräsen- 


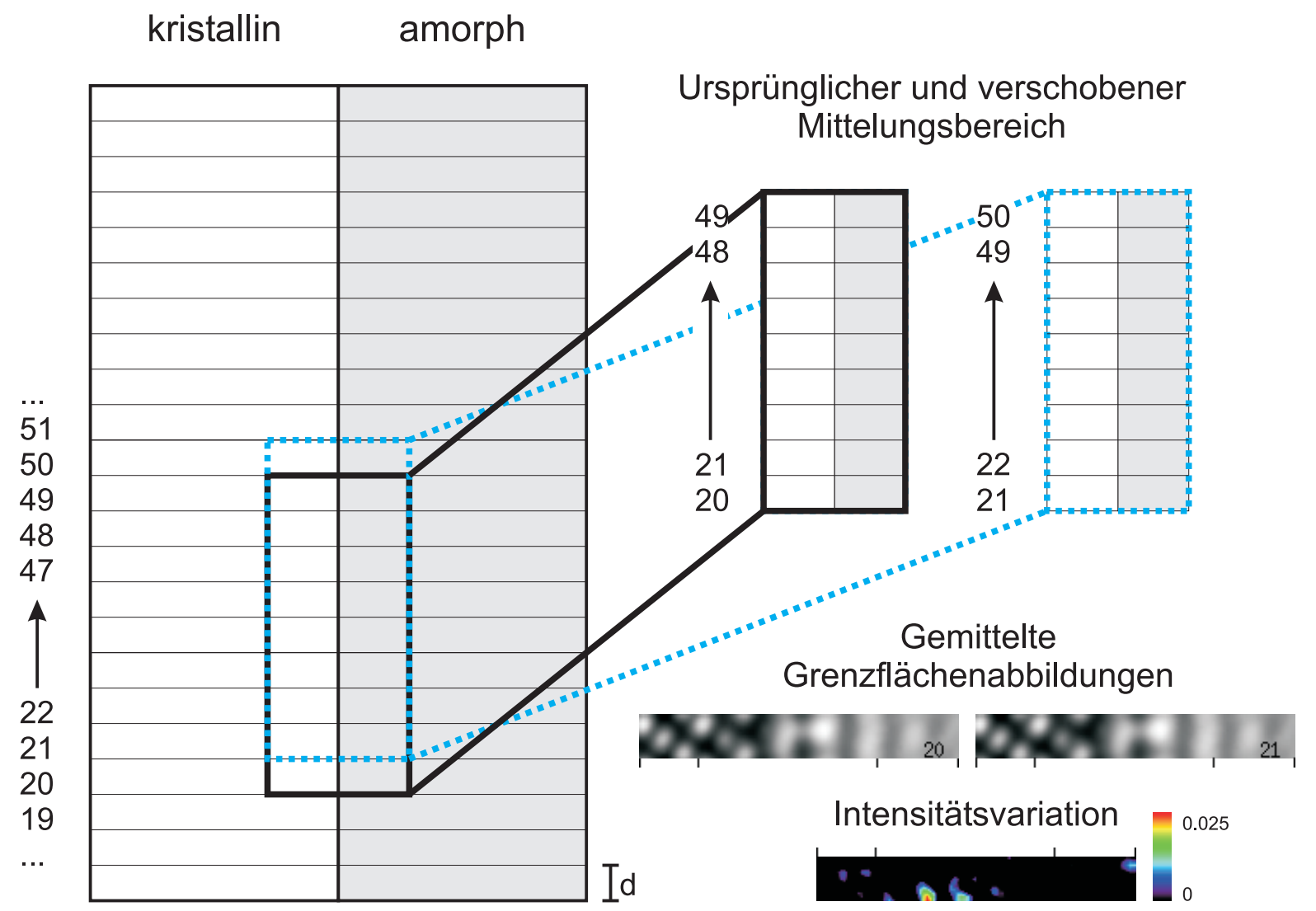

Abbildung 3.8 - Schemazeichnung für die Bestimmung der relativen Intensitätsvariationen zwischen zwei gemittelten Abbildungen. Genaue Erläuterungen im Text. 
tativ für diesen Bereich der Grenzfläche bei der in Schritt 1 gewählten Ausdehnung des Mittelungsbereiches.

5. Aufgrund von Unterschieden in der lokalen Struktur des Grenzflächenübergangs wird der Wert für die Intensitätsvariation von der Position des Mittelungsbereiches an der Grenzfläche abhängen. Es müssen also die Schritte 1-4 für alle möglichen Bereiche der Grenzfläche durchgeführt werden. Aus all diesen Werten wird schließlich der größte bestimmt. Er beschreibt die maximale Intensitätsvariation für die ausgewertete Grenzfläche mit der Größe $A=N d$ des Mittelungsbereiches über alle Abbildungen der Defokusserie.

Es ist zu erwarten, dass die auf diese Art und Weise bestimmte maximale Intensitätsvariation ihren größten Wert in dem Übergangsbereich zwischen dem kristallinen und amorphen Bereich der Probe erreichen wird. Aufgrund der Periodizität und langreichweitigen Ordnung des Kristalls variieren die Intensitäten des kristallinen Musters lediglich durch statistische Schwankungen. Die gemittelten Grenzflächenabbildungen - resultierend aus dem unverschobenen und dem verschobenen Mittelungsbereich - sollten hier also lediglich kleine Variationen aufweisen. Für den amorphen Probenteil gilt Ähnliches. Da das amorphe Muster keine ausgezeichnete Periodizität entlang der Grenzfläche besitzt, ergibt sich durch die Mittelung eine gleichmäßige Intensität in der gemittelten Grenzflächenabbildung. Die Intensitätsunterschiede zwischen den beiden Verteilungsfunktionen sollten hier ebenso klein sein. Im Übergangsbereich können sich aber entlang der Grenzfläche die atomare Struktur und somit die Muster in den elektronenmikroskopischen Abbildungen ändern, was sich grundlegend von den beiden eben besprochenen Situationen unterscheidet. In dem Fall sollte der Wert für die Intensitätsvariation hier größer sein.

Für die Quantifizierung der Intensitätsvariation wurde ein relatives Maß gewählt. Es bezeichnet den Unterschied der Intensität in äquivalenten Bildpunkten, normiert auf deren mittlere Intensität:

$$
P^{(J)}(N)=\max _{(x, y), k}\left\{\frac{\left|\left(I_{x y}^{(k)}\right)_{d}-\left(I_{x y}^{(k)}\right)\right|}{\frac{1}{2}\left[\left(I_{x y}^{(k)}\right)_{d}+\left(I_{x y}^{(k)}\right)\right]}\right\}
$$

Dabei bezeichnet $k$ die Abbildung innerhalb der Defokusserie und $\left(I_{x y}^{(k)}\right)$ bzw. $\left(I_{x y}^{(k)}\right)_{d}$ die Intensität im Punkt $(x, y)$ der gemittelten Grenzflächenabbildung, welche sich aus dem unverschobenen und um die Strecke $d$ verrückten Mittelungsbereich ergibt. In Anlehnung 
an Gleichung 3.1 in Abschnitt 3.2.1.2 ist dies:

$$
\begin{aligned}
\left(I_{x y}\right) & =\frac{1}{N} \sum_{n=J}^{J+N-1} I(x, y+n d) \\
\left(I_{x y}\right)_{d} & =\frac{1}{N} \sum_{n=J+1}^{J+N} I(x, y+n d)
\end{aligned}
$$

Der Index $J$ steht für die Position des Mittelungsgebietes an der Grenzfläche, die durch die Nummer des ersten Streifens innerhalb des Mittelungsgebietes festgelegt wird. In Abbildung 3.8 wäre somit $J=20$ für den unverschobenen und $J=21$ für den verschobenen Bereich. Der Wert für $J$ ist jeweils in den gemittelten Grenzflächenabbildungen angezeigt.

Wie oben bereits erwähnt, muss die Analyse für jede mögliche Position an der Grenzfläche durchgeführt werden, so dass sich schließlich für die maximale relative Intensitätsvariation entlang der Grenzfläche für alle Abbildungen der Defokusserie und für einen Mittelungsbereich der Ausdehnung $A=N d$ ergibt:

$$
P_{\max }(N)=\max _{J}\left\{P_{\max }^{(J)}(N)\right\}
$$

Da eine Verkleinerung des Mittelungsbereiches immer eine Erhöhung der Fehler in der Verteilungsfunktion (und umgekehrt) mit sich bringt, kann an dieser Stelle kein absoluter Grenzwert für die maximal zulässige relative Intensitätsvariation angegeben werden. Es bietet sich aber die Verwendung der in der Statistik üblichen Werte von 1\%, 5\% oder $10 \%$ für die Feststellung von Signifikanz an. Die Genauigkeit der APP-Näherung für die Größe des Mittelungsbereiches, die aus dieser Analyse folgt, kann wiederum durch Testsimulationen abgeschätzt werden.

\subsubsection{Fehlerbetrachtung}

Für die Beantwortung der Frage nach der strukturellen Homogenität entlang der Grenzfläche ist es notwendig, einen quantitativen Vergleich von Verteilungsfunktionen unterschiedlicher Bereiche der Grenzfläche durchzuführen. Dafür ist die Kenntnis der Genauigkeit dieser angepassten Funktionen von Bedeutung. Die möglichen Fehlerquellen können dabei in zwei Bereiche unterteilt werden: Zum einen sorgt die APP-Näherung selbst für einen systematischen Fehler, den BORGARDT ET AL. ${ }^{[13]}$ durch Simulationsrechnungen bereits auf $\leq 5 \%$ für die Parameter der zweidimensionalen Verteilungsfunktion abschätzen konnten. Zum anderen ist die Unsicherheit der Intensitätswerte in den gemittelten experimentellen Abbildungen zu nennen. Diese Fehler gehen nicht in die Simulationen ein, müssen aber berücksichtigt werden, da Unsicherheiten in der gemittelten Grenzflächenabbildung auch Unsicherheiten in $\rho(x, y)$ bedeuten. Varianzen in den Intensitäten 
der gemittelten Grenzflächenabbildung haben ihren Ursprung in Intensitätsvariationen in den zu mittelnden Streifen. Dafür können drei Gründe verantwortlich sein: systematische Fehler, statistische Schwankungen oder strukturelle Unterschiede innerhalb der Streifen. Systematische Fehler in den gemittelten experimentellen Intensitätswerten entstehen durch mögliche Probenveränderungen während der Aufnahme der Defokusserie oder durch Probenunterschiede im Bereich der Abbildung, da sich die Abbildungsbedingungen hierdurch lokal unterscheiden. Dies können z.B. Probenverbiegungen oder eine inhomogene Probendicke sein. Der statistische Fehler meint im Wesentlichen das so genannte Schrotrauschen bei der Bildaufzeichnung. Bei einer strukturell inhomogenen Grenzfläche entsteht durch die lateral unterschiedliche Reichweite des kristallperiodischen Kontrasts in den Streifen ein weiterer struktureller Beitrag zu den Varianzen der gemittelten Intensitätswerte im Übergangsbereich.

Üblicherweise wird der Fehler einer physikalischen Messung durch die wiederholte Durchführung eines Experimentes abgeschätzt. Jede Messung (im Folgenden als Datensatz $\mathcal{D}_{i}$ bezeichnet) kann dann an ein bestimmtes Modell angepasst werden und ergibt einen bestimmten Parametersatz $\vec{a}_{i}$ für dieses Modell. Dabei verteilen sich die Ergebnisse der Anpassungen aufgrund von leichten Unterschieden in den $\mathcal{D}_{i}$ um den experimentell in der Regel nicht zugänglichen wahren Parametersatz. Die Verteilung der $\vec{a}_{i}$ liefert schließlich eine Abschätzung der dem Modell zugrunde liegenden wahren Parameter.

Kann ein Experiment nicht hinreichend oft wiederholt werden, so bilden Monte-CarloSimulationen oft den einzig möglichen Weg zur Abschätzung von Fehlern in den Modellparametern. Dieser Weg wird auch in der vorliegenden Arbeit verfolgt und im Folgenden beschrieben.

\subsubsection{Monte-Carlo-Simulationen synthetischer Datensätze: Die Bootstrap-Methode}

Grundgedanke der Bootstrap-Methode ${ }^{[27-29]}$ ist die Erstellung so genannter ,synthetischer Datensätze“ $\mathcal{D}_{i}^{(S)}$. Jeder synthetische Datensatz repräsentiert dabei ein mögliches Ergebnis für einen experimentellen Datensatz. Das Verfahren nutzt dafür den experimentellen Datensatz $\mathcal{D}_{0}$, mit seinen $N$ Datenpunkten, um eine beliebige Anzahl $R$ synthetischer Datensätze $\mathcal{D}_{i}^{(S)}$ mit ebenfalls $N$ Datenpunkten zu generieren. Das Vorgehen dabei ist schlicht das zufällige Ziehen von Datenpunkten aus $\mathcal{D}_{0}$, wobei Mehrfachnennungen von Datenpunkten erlaubt sind. Durch die Möglichkeit der Mehrfachnennung unterscheiden sich die $\mathcal{D}_{i}^{(S)}$ von $\mathcal{D}_{0}$. Im Schnitt werden $\approx 37 \%$ der Datenpunkte durch mehrfach genannte Datenpunkte ersetzt. Jeder dieser synthetischen Datensätze kann nun an das Modell angepasst werden und ergibt einen synthetischen Parametersatz $\vec{a}_{r}^{(S)}$. Die Grundannahme der Bootstrap-Methode ist nun, dass sich die synthetischen Parametersätze $\vec{a}_{r}^{(S)}$ genauso um den experimentellen Parametersatz $\vec{a}_{0}$ verteilen wie eine Vielzahl experimentell bestimmter Parametersätze $\vec{a}_{i}$ um den wahren Parametersatz. Die Richtigkeit dieser Annahme wurde bereits vielfach belegt ${ }^{[28]}$. Die Anzahl der zu generierenden syntheti- 
Originaler Datensatz

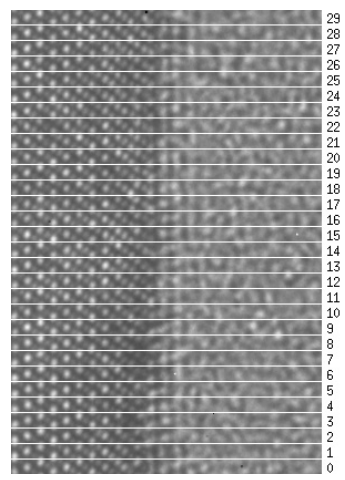

In seine Datenpunkte zerlegter originaler Datensatz, i.e. die einzelnen Streifen

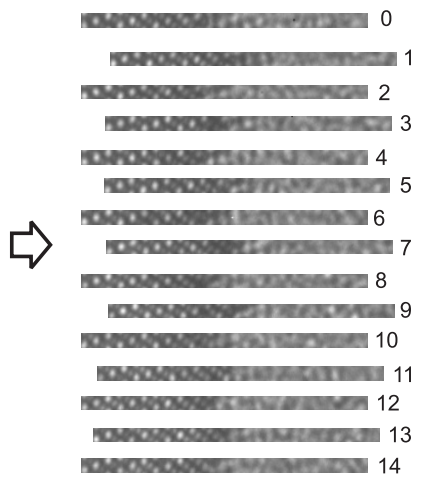

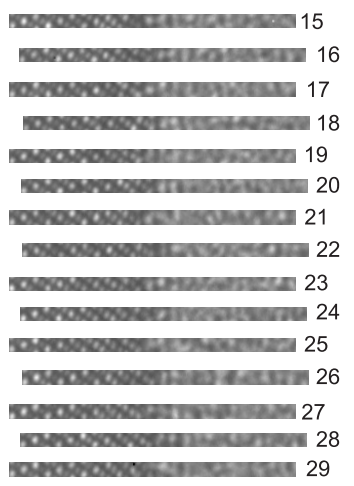

Synthetischer Datensatz



Abbildung 3.9 - Graphische Darstellung der Konstruktion synthetischer Datensätze. Links: Ausgangspunkt ist der experimentelle Datensatz, also das in Streifen unterteilte Mittelungsgebiet der Grenzfläche. Jeweils rechts neben den Streifen ist die Streifennummer angezeigt. Mitte: Dieser Datensatz wird in seine Datenpunkte, also die einzelnen Streifen, zerlegt. Rechts: Aus diesem Datenpool wird durch wiederholtes Ziehen eine neue Zusammenstellung von Streifen, ein synthetischer Datensatz, erstellt. Mehrfachnennungen einzelner Streifen sind dabei erlaubt. Da über alle Streifen später gemittelt wird, ist die Reihenfolge der Ziehung unerheblich. Aufgrund der Mehrfachnennungen fehlen im synthetischen Datensatz hier die Streifen: 5,7,9,10,11,12,14,20,22,23,26 \& 28, also $40 \%$.

schen Datensätze wird dabei mit 25 bis 200 angegeben. Einen genauen Überblick über die Methode bieten EFroN ${ }^{[28]}$ oder PREss ET AL. ${ }^{[29]}$.

In dem in der vorliegenden Arbeit behandelten Fall besteht der experimentelle Datensatz aus den $N$ Streifen des Mittelungsgebietes. Durch das zufällige Auswählen von $N$ Streifen entsteht nun ein synthetischer Datensatz, wobei jeder Streifen beliebig oft gewählt werden kann. Die Reihenfolge, in der die Streifen gezogen werden, ist hier nicht von Bedeutung, da später für die Berechnung der mittleren Grenzflächenabbildung über alle Streifen gemittelt wird. Abbildung 3.9 illustriert das Vorgehen.

Abbildung 3.10 zeigt zwei synthetische Datensätze, den experimentellen Datensatz und deren gemittelte Abbildungen sowie die Profile der atomaren Dichte im Vergleich. Es ist deutlich zu erkennen, dass sich die gemittelten Grenzflächenabbildungen der experimentellen und synthetischen Datensätze nicht stark, d.h. für das Auge deutlich sichtbar, unterscheiden.

Jede gemittelte Grenzflächenabbildung aus den $R$ synthetischen Datensätzen wurde mittels der iterativen Bildanpassung untersucht und lieferte jeweils eine neue zweidimensionale Verteilungsfunktion $\rho^{(r)}(x, y)$ mit $r=1,2, \ldots, R$. Aus den $R$ Verteilungsfunktionen ergibt sich für jeden Bildpunkt eine Intensitätsverteilung und somit auch eine 
(a)

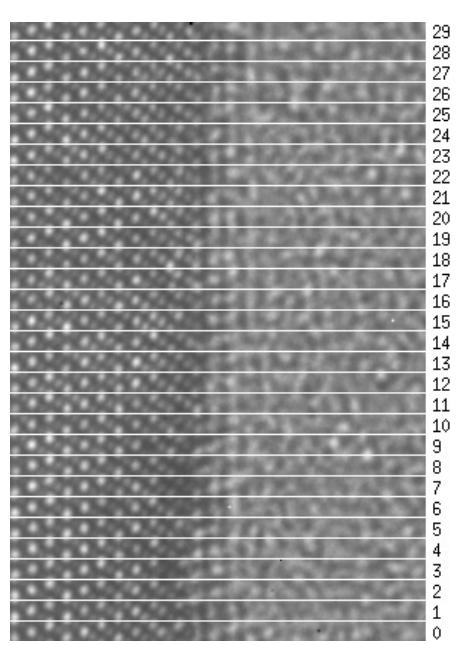

Synthetischer Datensatz \#1

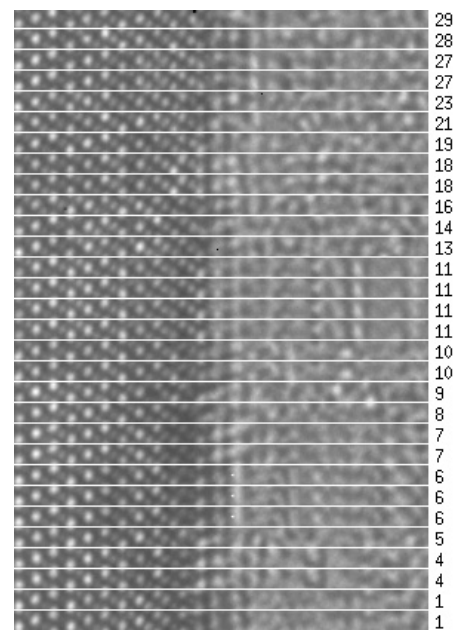

Synthetischer Datensatz \#2

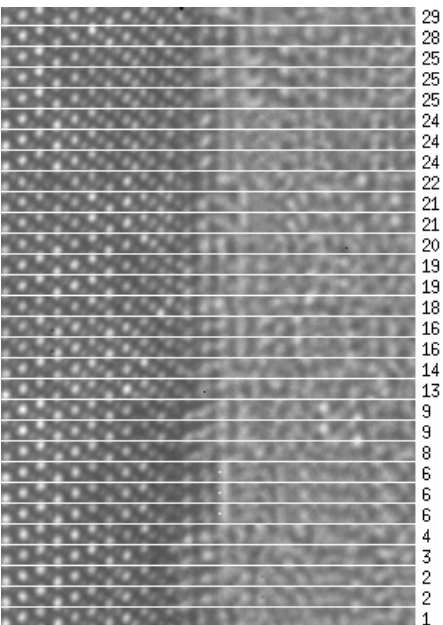

(b) Ursprünglicher Datensatz
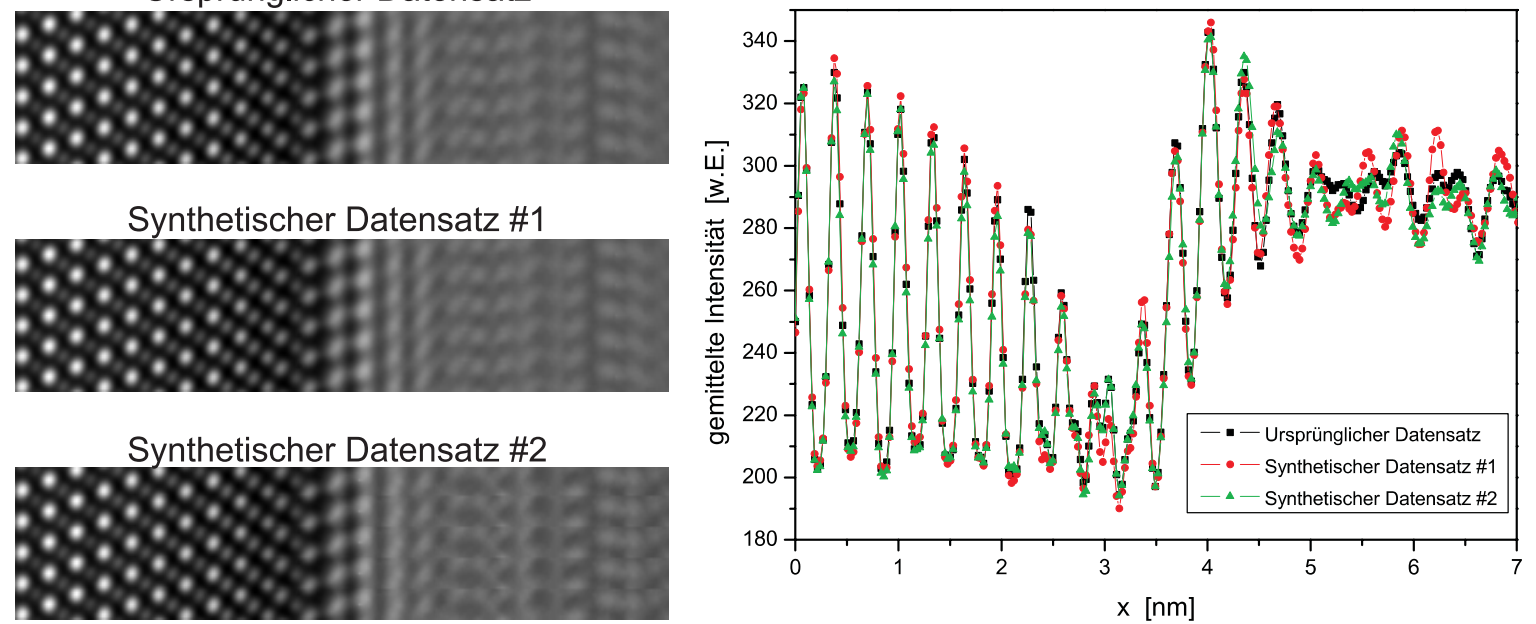

Abbildung 3.10 - (a) Synthetische Datensätze. Die linke Seite zeigt den experimentellen Datensatz bestehend aus den 30 Streifen 0 bis 29. In der Mitte und rechts ist jeweils ein möglicher synthetischer Datensatz dargestellt. Jeweils rechts von den Abbildungen sind die Nummern der Streifen angezeigt. Die Reihenfolge der Streifen spielt keine Rolle. (b) Vergleich der mittleren Grenzflächenabbildungen und Profile des experimentellen Datensatzes mit denen der synthetischen Datensätze. Links: Die drei gemittelten Grenzflächenabbildungen. Rechts: Vergleich der Intensitätsprofile. Es ist gut zu erkennen, dass sich die gemittelten Grenzflächenabbildungen der synthetischen Datensätze in diesem Beispiel nicht wesentlich von der gemittelten Grenzflächenabbildung des ursprünglichen Datensatzes unterscheiden. Lediglich fern der Grenzfläche gibt es Unterschiede, die sich aber durch die geringere Anzahl der unterschiedlichen Streifen und somit durch die reduzierte Statistik erklären lassen. 
Standardabweichung $\sigma_{\rho}(x, y)$ :

$$
\sigma_{\rho}(x, y)=\sqrt{\frac{1}{R-1} \sum_{r=1}^{R}\left(\rho^{(r)}(x, y)-\frac{1}{R} \sum_{r^{\prime}=1}^{R} \rho^{\left(r^{\prime}\right)}(x, y)\right)^{2}}
$$

Dementsprechend können auch Fehler für das Profil der atomaren Dichte aus den $R$ synthetischen Profilen $\bar{\rho}^{(r)}(x)$ berechnet werden:

$$
\bar{\sigma}_{\rho}(x)=\sqrt{\frac{1}{R-1} \sum_{r=1}^{R}\left(\bar{\rho}^{(r)}(x)-\frac{1}{R} \sum_{r^{\prime}=1}^{R} \bar{\rho}^{\left(r^{\prime}\right)}(x)\right)^{2}}
$$

Durch diese Fehlerabschätzung ist es erstmals möglich, Unterschiede zwischen zwei Verteilungsfunktionen, die der Anpassung zweier unterschiedlicher Bereiche der Grenzfläche entstammen, in äquivalenten Punkten auf ihre Signifikanz hin zu untersuchen. 


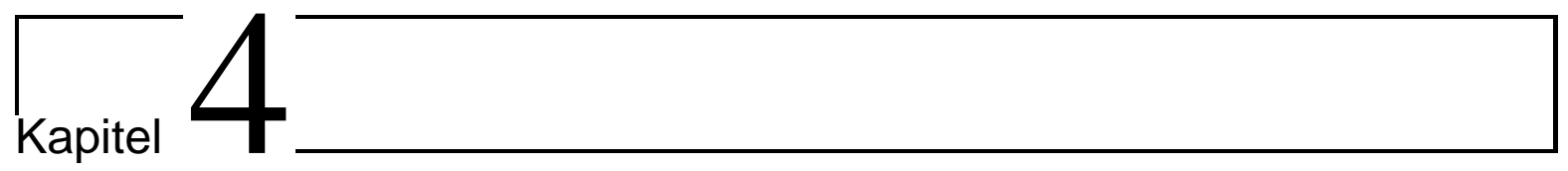

\section{Ergebnisse}

\section{Inhaltsangabe}

4.1 Struktureller Übergang an den Grenzflächen a-Si/c-Si und

a-Ge/c-Si ..................... 51

4.1.1 Experimentelle gemittelte Abbildungen . . . . . . . . . . . . . 52

4.1.2 Simulation gemittelter Abbildungen . . . . . . . . . . . . 55

4.2 Laterale Homogenität der zweidimensionalen Verteilungsfunktion ................... 71

4.2 .1 a-Ge/c-Si-Probe . . . . . . . . . . . . . . . 71

4.2 .2 a-Si/c-Si-Probe . . . . . . . . . . . . . . . . . . . 75

In diesem Kapitel sollen die Ergebnisse der Analyse der a-Ge/c-Si- bzw. a-Si/c-Si-Probe mittels der in Kapitel 3 dargestellten Verfahren vorgestellt werden. Dabei wird in Abschnitt 4.1 der strukturelle Übergang zwischen den Materialien und in Abschnitt 4.2 die Frage nach der lateralen Homogenität der zweidimensionalen Verteilungsfunktion entlang der Grenzfläche genauer betrachtet.

\subsection{Struktureller Übergang an den Grenzflächen a-Si/c-Si und a-Ge/c-Si}

In diesem Abschnitt soll der strukturelle Übergang zwischen amorphem Silizium und kristallinem Silizium sowie amorphem Germanium und kristallinem Silizium mithilfe der in Kapitel 3 beschriebenen Vorgehensweise untersucht werden. In Abschnitt 4.1.1 werden dazu die gemittelten experimentellen Abbildungen der Defokusserien beider Proben und in Abschnitt 4.1.2 die simulierten Abbildungen sowie die numerisch angepassten zweidimensionalen Verteilungsfunktionen betrachtet. 


\begin{tabular}{cccc}
\hline \hline \multirow{2}{*}{ Probe } & Startdefokus & Defokusschrittweite & Probendicke \\
& $\Delta f_{1}$ & $\delta \Delta f$ & $t$ \\
\hline $\mathrm{a}-\mathrm{Si} / \mathrm{c}-\mathrm{Si}$ & $37 \mathrm{~nm}$ & $-6.7 \mathrm{~nm}$ & $9.6(4) \mathrm{nm} \ldots 11.1(4) \mathrm{nm}$ \\
$\mathrm{a}-\mathrm{Ge} / \mathrm{c}-\mathrm{Si}$ & $32 \mathrm{~nm}$ & $-6.7 \mathrm{~nm}$ & $13.8(4) \mathrm{nm}$ \\
\hline \hline
\end{tabular}

Tabelle 4.1 - Ergebnisse für die Bestimmung des Startdefokus $\left(\Delta f_{1}\right)$, der Defokusschrittweite $(\delta \Delta f)$ und der Probendicke $(t)$ anhand des Vergleichs von simulierten und experimentellen Gitterabbildungen einer Defokusserie.

Tabelle 4.2 - Ergebnisse der Bestimmung der Probendicke und deren laterale Position für die a-Si/c-SiProbe (s. auch Abb. 4.1).

\begin{tabular}{lccc}
\hline \hline Streifen & $0 \rightarrow 29$ & $19 \rightarrow 48$ & $39 \rightarrow 68$ \\
\hline Probendicke $t$ & $9.6(4) \mathrm{nm}$ & $10.4(4) \mathrm{nm}$ & $11.1(4) \mathrm{nm}$ \\
\hline \hline
\end{tabular}

\subsubsection{Experimentelle gemittelte Abbildungen}

Entsprechend der in den Abschnitten 3.2 und 3.2.3.2 vorgestellten Verfahren wurden die Drift innerhalb der experimentellen Defokusserien korrigiert (Abschnitt 3.2.1.1), die mittleren Grenzflächenabbildungen berechnet (Abschnitt 3.2.1.2) sowie Probendicke $(t)$, Startdefokus $\left(\Delta f_{1}\right)$ und Defokusschrittweite $(\delta \Delta f)$ bestimmt (Abschnitt 3.2.3.2). In diesem Abschnitt sollen nun die Ergebnisse zusammengefasst werden.

Die Defokusserie der a-Si/c-Si-Probe wurde in 71 Streifen und die der a-Ge/c-SiProbe in 70 Streifen senkrecht zur Grenzfläche eingeteilt. Da die Gesamtdrift bei der Germanium-Probe größer war, ist der gemeinsame Probenbereich der gesamten Defokusserie kleiner und aus diesem Grund die Anzahl der Streifen geringer.

Da die exakte Streifenhöhe über die Defokusserien im Bereich von 12 bis 13 Bildpunkten leicht variiert, wurden alle Streifen mittels bilinearer Interpolation auf eine einheitliche Höhe von 13 Bildpunkten gerechnet. Die Streifenbreite beträgt 280 Bildpunkte. Die Abbildungen 4.1 (a-Si/c-Si) und 4.2 (a-Ge/c-Si) zeigen jeweils eine in Streifen unterteilte experimentelle Abbildung beider Defokusserien bei der Einstellung des minimalen Kontrasts.

Tabelle 4.1 fasst die Ergebnisse für die Bestimmung der Probendicke, des Startdefokus und der Defokusschrittweite für beide Defokusserien zusammen. Die Werte, die bereits von PLIKAT ${ }^{[24]}$ für die Defokusserie der a-Ge/c-Si-Probe gefunden wurden $\left(\Delta f_{1}=40 \mathrm{~nm}\right.$, $\delta \Delta f=7 \mathrm{~nm}$ und $t=12.4(5) \mathrm{nm})$, konnten durch den in Abschnitt 3.2.3.2 beschriebenen iterativen Vergleich von experimentellen mit simulierten Gitterabbildungen von BoRGARDT ET AL. nochmals präzisiert werden ${ }^{[11,13]}$.

Im Gegensatz zu der a-Ge/c-Si-Probe variieren die Werte für die Probendicke im Bereich der Grenzfläche der a-Si/c-Si-Probe in Abhängigkeit von der Position des Auswertungsbereiches zwischen 9.6nm und 11.1nm (siehe auch Tabelle 4.2). Dies ist besonders gut in den experimentellen Abbildungen anhand der sich verändernden Muster 
4.1 Struktureller Übergang an den Grenzflächen a-Si/c-Si und a-Ge/c-Si

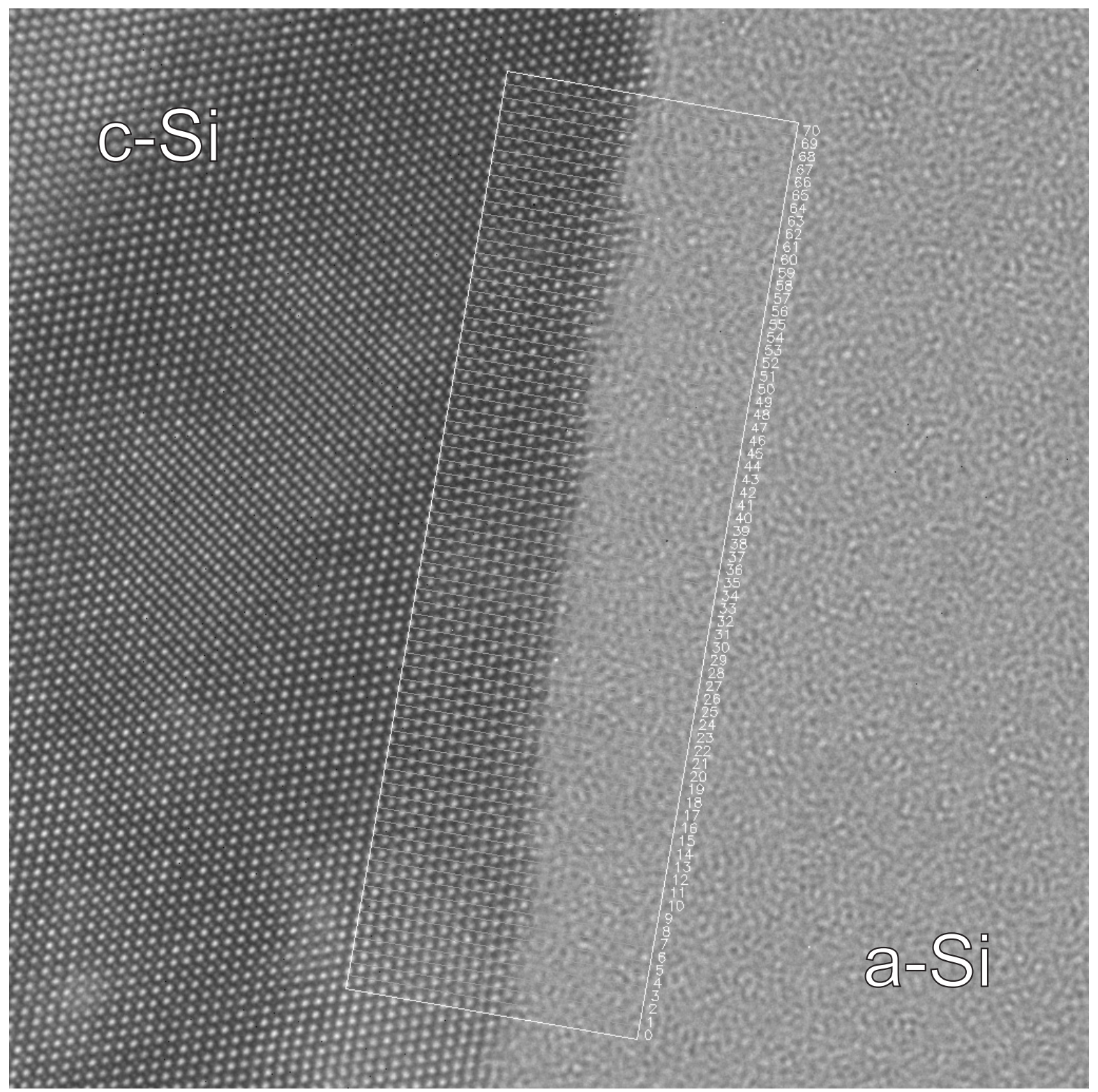

Abbildung 4.1 - Abbildung der Grenzfläche zwischen amorphem und kristallinem Silizium und deren Einteilung entsprechend der in Abschnitt 3.2.1.2 besprochenen Vorgehensweise in 71 Streifen senkrecht zur Grenzfläche. Der Defokuswert ist $\Delta f=-23.3 \mathrm{~nm}$. 


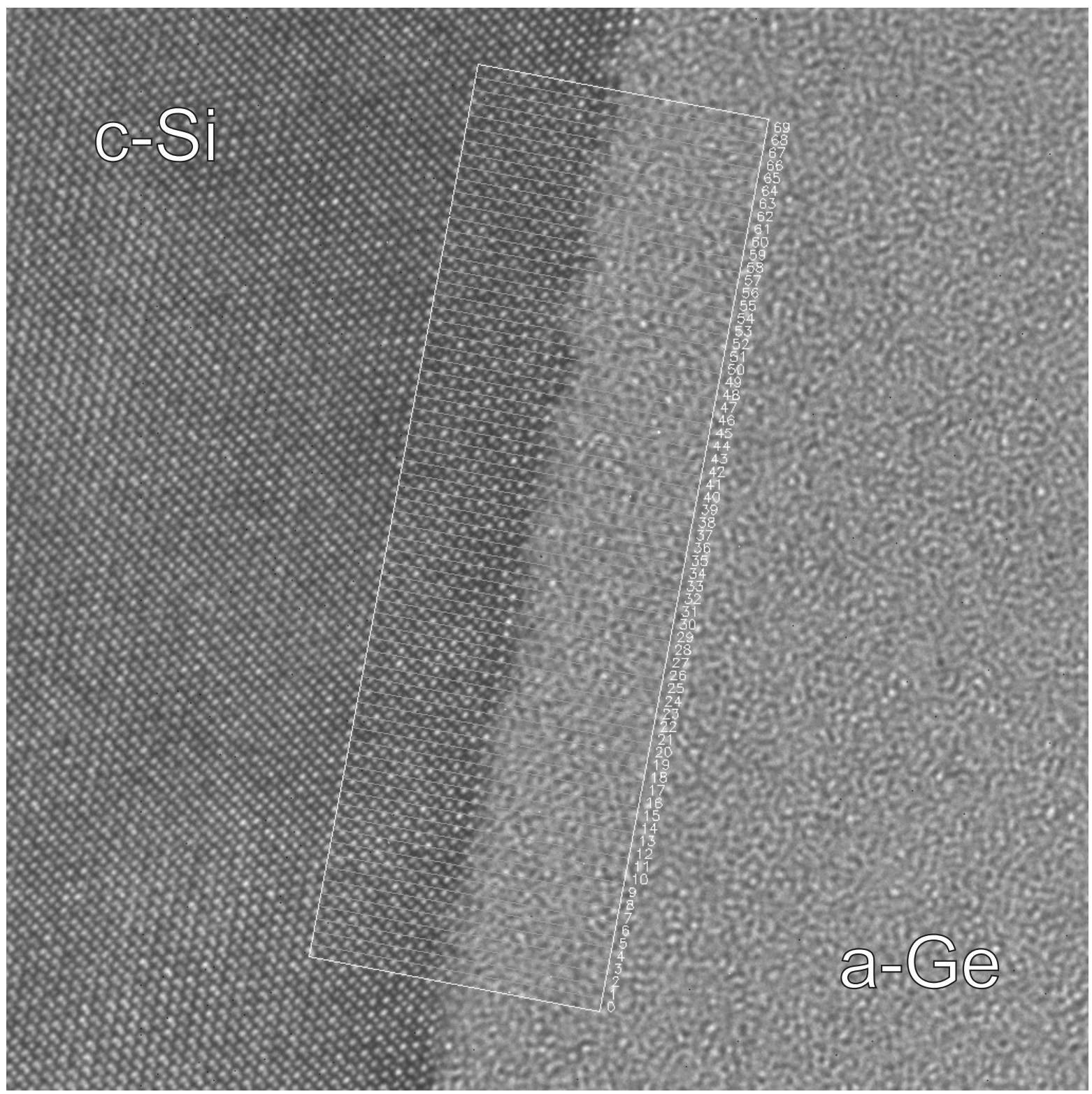

Abbildung 4.2 - Abbildung der Grenzfläche zwischen amorphem Germanium und kristallinem Silizium und deren Einteilung in siebzig Streifen senkrecht zur Grenzfläche. Der Defokuswert beträgt $\Delta f=-21.6 \mathrm{~nm}$. 
in der Gitterabbildung entlang der Grenzfläche zu erkennen. Die Unterschiede in der Probendicke bedeuten, dass diese Probe entlang der Grenzfläche eine Keilform mit einer Steigung von $\approx 3.7^{\circ}$ aufweist. Im Gegensatz dazu ist die a-Ge/c-Si-Probe über den gesamten Auswertungsbereich an der Grenzfläche mit $t=13.8(4)$ nm gleichmäßig dick. Lediglich senkrecht zur Grenzfläche in Richtung des Kristalls existiert ein Gradient in der Probendicke mit einem Keilwinkel von $24(5)^{\circ}$, was bereits von PLIKAT ${ }^{[24]}$ diskutiert wurde.

Abbildung 4.3 zeigt die gemittelten Grenzflächenabbildungen der a-Si/c-Si-Defokusserie, die zur deutlicheren Sichtbarkeit viermal untereinander dargestellt sind. In diesem Fall wurde der mittlere Bereich der Abbildung (Streifen 19 bis 48) zur Mittelung benutzt. Hingegen konnte aufgrund der einheitlichen Probendicke der a-Ge/c-Si-Probe hier über alle 70 Streifen gemittelt werden. Abbildung 4.4 zeigt das Ergebnis.

Wie bereits in Abschnitt 3.1 diskutiert, ist in den gemittelten Abbildungen jeweils kristalliner Kontrast im amorphen Teil der Probe zu erkennen, der bei einem Defokuswert von $\Delta f=-23.3 \mathrm{~nm}$ innerhalb der Defokusserie der a-Si/c-Si-Probe und bei $\Delta f=-21.6 \mathrm{~nm}$ innerhalb der Defokusserie der a-Ge/c-Si-Probe am geringsten ist. Die Gründe hierfür, die durch das Substrat induzierte Ordnung in der Nähe der Grenzfläche und die defokusabhängige Delokalisierung, wurden bereits in Abschnitt 3.1 diskutiert.

\subsubsection{Simulation gemittelter Abbildungen}

Entsprechend der in Abschnitt 3.2.2 diskutierten Vorgehensweise wurden dann die im letzten Abschnitt berechneten mittleren Grenzflächenabbildungen mithilfe der MultisliceMethode innerhalb der APP-Näherung simuliert.

Die Kantenlängen der quaderförmigen Superzelle waren für beide Proben durch $a=$ $9.4062 \mathrm{~nm}, b=2 d=0.6651 \mathrm{~nm}$ und $c=0.3840 \mathrm{~nm}$ festgelegt. Die Werte für $b$ und $c$ entsprechen der Größe der Elementarzelle des Siliziums entlang der [1 10$]$ - und [1̄12]Richtung. In die Simulationen wurden des Weiteren die Defokuswerte für die experimentellen Abbildungen und die Probendicke einbezogen, deren Werte im letzten Abschnitt 4.1 .1 bestimmt wurden (s. Tabelle 4.1 und 4.2). Als Debye-Waller-Faktor wurde $0.5 \AA^{2[115]}$, für die sphärische Aberration $C_{S}=0.48 \mathrm{~mm}$ und eine Apertur von $24 \mathrm{mrad}$, entsprechend der im Experiment benutzten Blende, verwendet. Alle Effekte, die Elektronen aus der kohärenten Vielfachstreuung entfernen (z.B. inelastische Streuung, Absorption), wurden in den Simulationen durch den Imaginärteil des Streupotentials phänomenologisch berücksichtigt. Dabei wurde die Annahme gemacht, dass dieser Anteil proportional zum Realteil ist. Für den kristallinen Probenteil wurde ein Verhältnis von 0.05 und für den amorphen Teil ein Verhältnis von 0.04 angenommen ${ }^{[13]}$. Mithilfe dieser Werte konnte das Verhältnis der mittleren Intensitäten im kristallinen und amorphen Probenteil der experimentellen Abbildungen gut durch die Simulationen wiedergegeben werden.

Um über den gesamten Vergleichsbereich eine einheitliche Probendicke zu gewähr- 


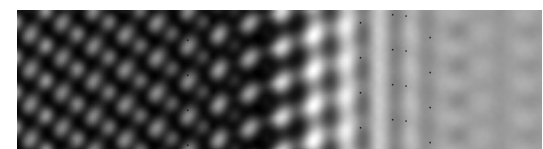

$30.3 \mathrm{~nm}$

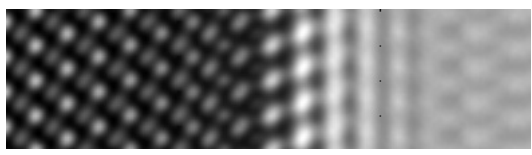

23.6nm

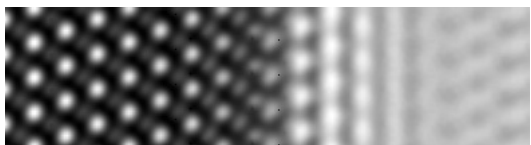

$16.9 \mathrm{~nm}$

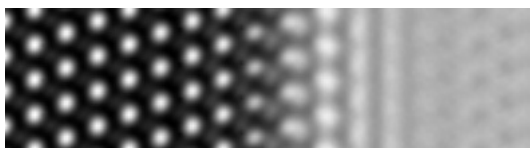

$10.2 \mathrm{~nm}$

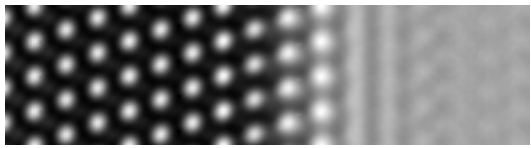

$3.5 \mathrm{~nm}$
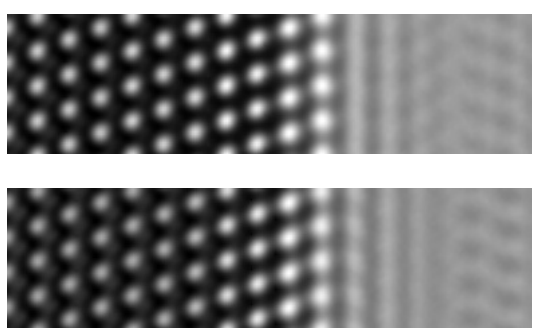

$-9.9 \mathrm{~nm}$

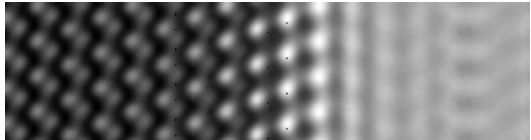

$-16.6 n m$

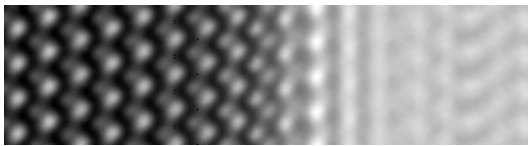

$-23.3 n m$

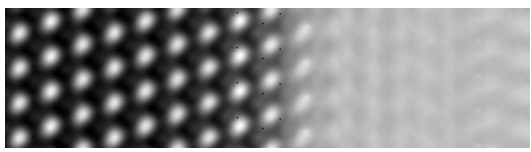

$-30 n m$

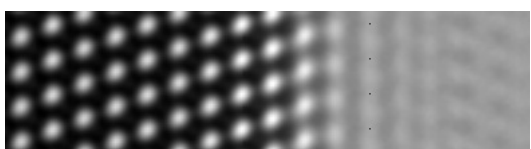

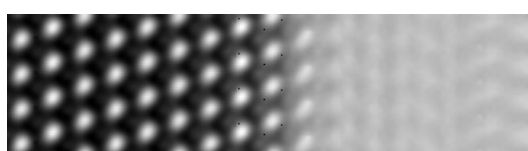

$-23.3 n m$

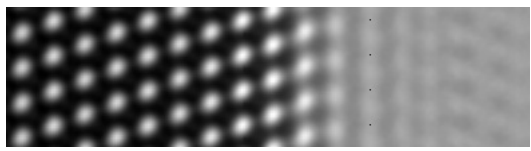

$-30 n m$



$-36.7 \mathrm{~nm}$

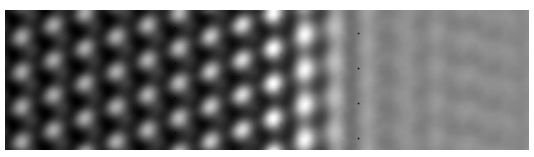

$-43.3 n m$

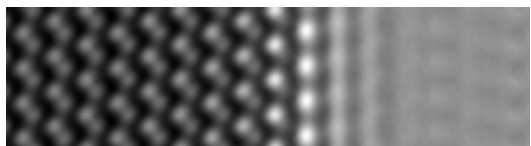

$-50.1 \mathrm{~nm}$



$-56.8 n m$

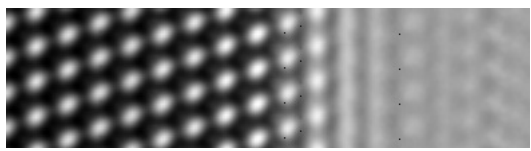

$-63.5 n m$

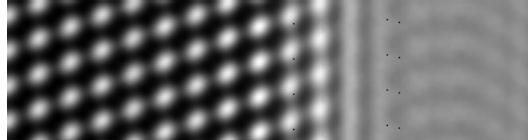

$-70.2 \mathrm{~nm}$

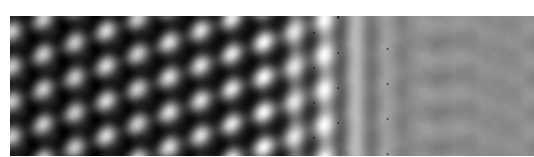

$-76.9 \mathrm{~nm}$

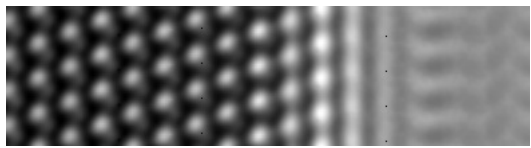

$-83.6 n m$

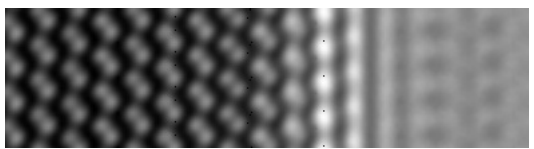

$-90.3 \mathrm{~nm}$

Abbildung 4.3 - Gemittelte Grenzflächenabbildungen der Defokusserie der a-Si/c-Si-Probe. Neben der Abbildung ist jeweils der Defokuswert angezeigt. Die Abbildungen zu den Defoki $\Delta f=-23.3 \mathrm{~nm}$ und $\Delta f=-30 \mathrm{~nm}$ sind in der rechten Spalte noch einmal dargestellt. 

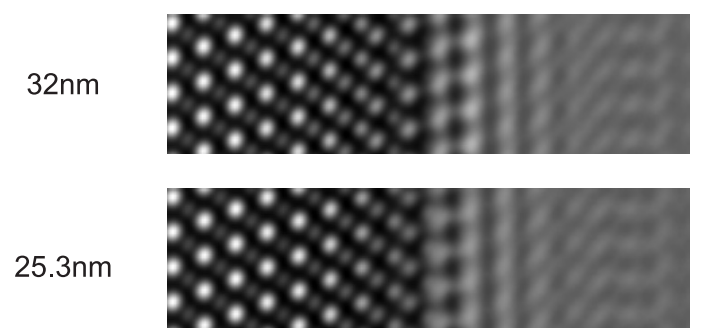

$18.6 \mathrm{~nm}$

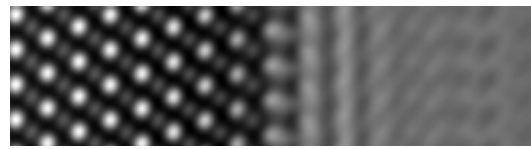

$11.9 \mathrm{~nm}$



$5.2 \mathrm{~nm}$

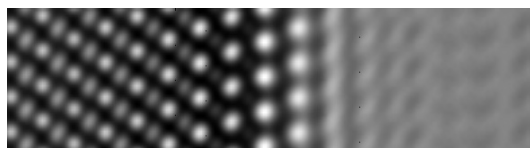

$-1.5 \mathrm{~nm}$
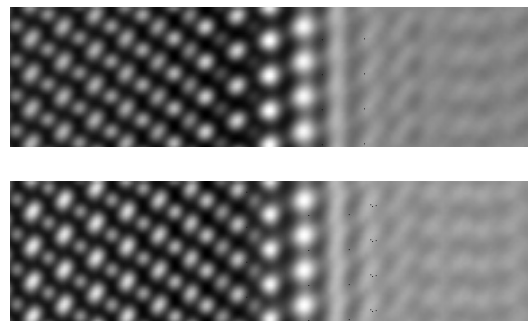

$-14.9 n m$

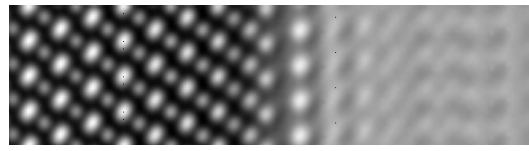

$-21.6 n m$



$-28.3 n m$
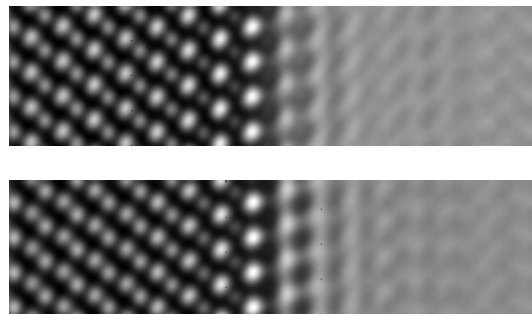

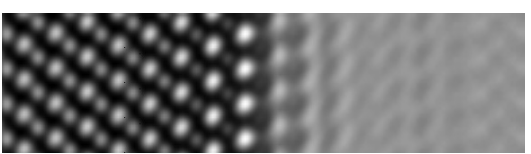

$-28.3 n m$

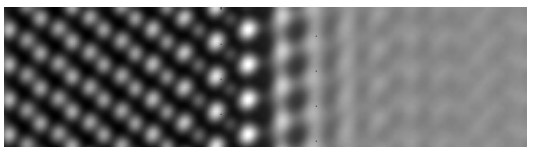

$-35 n m$

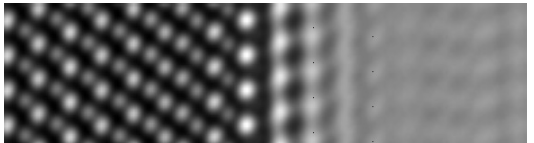

$-41.7 n m$

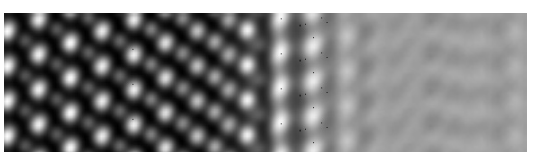

$-48.4 n m$

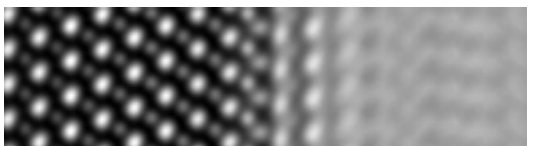

$-55.1 \mathrm{~nm}$

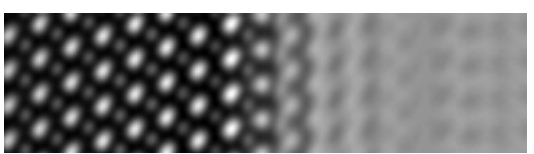

$-61.8 \mathrm{~nm}$

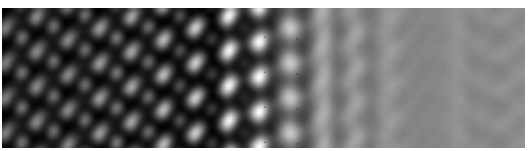

$-68.5 n m$

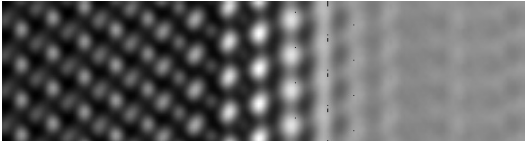

$-75.2 \mathrm{~nm}$

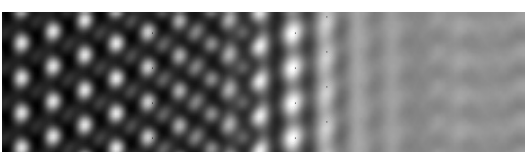

$-81.9 n m$



$-88.6 n m$

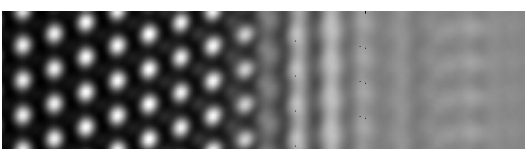

$-95.3 n m$

Abbildung 4.4 - Gemittelte Grenzflächenabbildungen der Defokusserie der a-Ge/c-Si-Probe. Neben der Abbildung ist jeweils der Defokuswert angezeigt. Die Abbildungen zu den Defoki $\Delta f=-28.3 \mathrm{~nm}$ und $\Delta f=-35 \mathrm{~nm}$ sind in der rechten Spalte noch einmal dargestellt. 
leisten, wurde die Berechnung des R-Faktors bei der Defokusserie der a-Ge/c-Si-Probe innerhalb der 9.4nm großen Superzelle für den Bereich 4.3nm $\leq x \leq 6.8 \mathrm{~nm}$ durchgeführt und bei der Defokusserie der a-Si/c-Si-Probe für 3.7nm $\leq x \leq 7.1 \mathrm{~nm}$. Als Anpassungsalgorithmus für die iterative Bildanpassung wurde die Simplex-Methode von NELDER UND MEAD verwendet ${ }^{[29,116]}$.

Wie bereits in Abschnitt 3.2.2.3 erwähnt, gibt es zwei mögliche Bindungskonfigurationen für die erste atomare Lage des amorphen Materials an den Kristall: Sie kann eine oder drei Rückbindungen aufweisen. Für die a-Ge/c-Si-Probe haben BorGARDT ET AL. bereits gezeigt ${ }^{[13]}$, dass die besten Anpassungen erreicht werden, wenn die erste atomare Lage des amorphen Materials mit einer Rückbindung an den Kristall gebunden ist. Für die a-Si/c-Si-Probe ergab sich dasselbe Ergebnis.

\subsubsection{Bestimmung der notwendigen Parameteranzahl zur Konstruktion der zweidimensionalen Verteilungsfunktion}

In Abschnitt 3.2.2.3 wurde bereits der Einfluss einer erhöhten Anzahl von Parametern auf die Konstruktion der zweidimensionalen Verteilungsfunktion und die daraus resultierende bessere Beschreibung der grenzflächeninduzierten Ordnung diskutiert. In diesem Abschnitt wird die Frage behandelt, wie viele Parameter mindestens nötig sind, um eine korrekte Beschreibung zu erreichen. Zu diesem Zweck wurde die iterative Bildanpassung für die Defokusserie der a-Ge/c-Si-Probe mit zwei, sechs und acht Parametern und für die a-Si/c-Si-Probe mit 6 und 8 Parametern für $\rho(x, y)$ durchgeführt, und die experimentellen gemittelten Abbildungen wurden mit den simulierten mittels des R-Faktors verglichen. Abbildung 4.5 zeigt das Ergebnis dieser Rechnungen.

Die entsprechenden R-Faktoren waren für die Defokusserie der a-Ge/c-Si-Probe: $\mathrm{R}_{2}=$ $248, R_{6}=189$ und $R_{8}=190$ und für die der a-Si/c-Si-Probe: $R_{6}=156$ und $R_{8}=155^{*}$. Während die Werte für sechs und acht Parameter praktisch gleich sind, ergibt sich gegenüber der Verwendung von zwei Parametern eine Verbesserung um $\approx 30 \%$. Die Erhöhung der Parameteranzahl führt somit zu einer deutlich besseren Übereinstimmung zwischen den experimentellen und simulierten gemittelten Abbildungen der Defokusserie (vgl. Abschnitt 3.2.2.3).

Der Effekt auf die zweidimensionale Verteilungsfunktion $\rho(x, y)$ wird in Abbildung 4.5(a) deutlich. Der Übergang von 2 zu 6 Parametern führt zu Änderungen vor allem in den Atomlagen 2 bis 4 , was mit dem in Abschnitt 3.2.2.3 beschriebenen Konstruktionsmodell übereinstimmt. Die weitere Erhöhung auf 8 Parameter führt zu Variationen jenseits der 4. Atomlage. In diesem Bereich ist die grenzflächeninduzierte Ordnung im amorphen Material jedoch so weit abgeklungen, dass keine signifikant bessere Übereinstimmung zwischen experimenteller und simulierter Defokusserie erreicht wird.

*Für die Untersuchungen in diesem Abschnitt wurde der R-Faktor analog zu der a-Ge/c-Si-Probe für den Bereich $4.3 \mathrm{~nm} \leq x \leq 6.8 \mathrm{~nm}$ berechnet. 
(a)
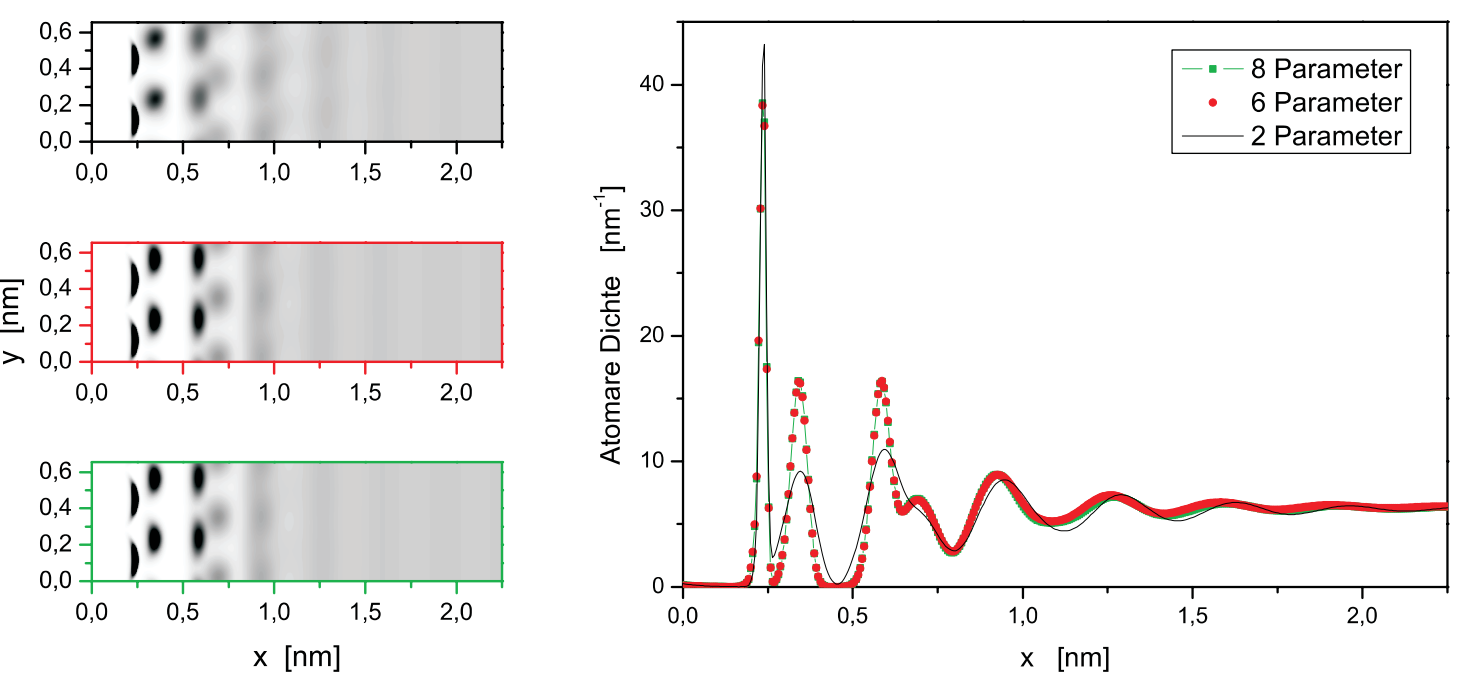

(b)


Abbildung 4.5 - Vergleich der zweidimensionalen Verteilungsfunktionen $\rho(x, y)$ (links), die mit zwei (schwarz), sechs (rot) und acht (grün) Parametern konstruiert wurden, und deren eindimensionale Profile (rechts) für die Defokusserie der (a) a-Ge/c-Si- und (b) a-Si/c-Si-Probe. Es ist deutlich zu erkennen, dass zwischen dem Profil mit zwei und dem mit sechs bzw. acht Parametern ein deutlicher Unterschied besteht. Im Gegensatz dazu unterscheiden sich die Profile mit sechs und acht Parametern nur unwesentlich. Die Variationen sind hier so klein, dass sie bei der iterativen Bildanpassung nicht weiter ins Gewicht fallen und keine signifikant unterschiedlichen Ergebnisse liefern. 


\subsubsection{2 a-Si/c-Si-Probe}

Die Abbildungen 4.6 und 4.7 zeigen die Ergebnisse der iterativen Bildanpassung für die a-Si/c-Si-Probe anhand des Profilvergleichs, während Abbildung 4.8 die experimentellen und simulierten gemittelten Grenzflächenabbildungen vergleicht. Die zweidimensionale Verteilungsfunktion für die mittlere Atomverteilung des amorphen Siliziums wurde, wie im letzten Abschnitt diskutiert, mit sechs Parametern konstruiert.

Es ist eine gute Übereinstimmung der Profilverläufe zu erkennen. Aufgrund des in Kapitel 2.3.3 diskutierten Stobbs-Faktors sind die experimentell beobachteten Kontraste kleiner als die simulierten. Des Weiteren ist zu sehen, dass im kristallinen Probenbereich die kontrastschwachen doppeltperiodischen Maxima in den Profilen der experimentellen Abbildungen im Gegensatz zu den Profilen der simulierten Abbildungen (z.B. für den Defokuswert $\Delta f=-3.2 \mathrm{~nm}$ ) nicht vorhanden sind, obwohl sie in den zweidimensionalen Grenzflächenabbildungen deutlich hervortreten. Dies kann durch eine leichte Probenverbiegung erklärt werden, die in den experimentellen gemittelten Abbildungen dazu führt, dass die für eine Kontrastumkehr charakteristischen doppeltperiodischen Maxima leicht gegeneinander verschoben sind (s. Abb. 4.8 beim Defokuswert $\Delta f=-16.6 \mathrm{~nm}$ in den blauen Kreisen). Bei der Berechnung der eindimensionalen Profile werden sie während der Projektion überdeckt. Diese Asymmetrie wird in den Simulationen nicht berücksichtigt.

Um die Position der Grenzfläche zu bestimmen, wurde, ausgehend von $x_{\text {Grenz }}=$ $4.15 \mathrm{~nm}$, dessen Position sukzessive um jeweils eine Atomdoppellage, i.e. $d_{x}=0.31354 \mathrm{~nm}$, in Richtung des amorphen Bereiches bis $\mathrm{zu} x_{\mathrm{Grenz}}=5.1 \mathrm{~nm}$ verschoben und die iterative Bildanpassung durchgeführt. Um die induzierte Ordnung, deren Reichweite von der Position der angenommenen Grenzfläche abhängt, korrekt beschreiben zu können, wurde die zweidimensionale Verteilungsfunktion für die Positionen $x_{\mathrm{Grenz}}=5.1 \mathrm{~nm}$ und $4.78 \mathrm{~nm}$ mit 6 Parametern und für die Positionen $x_{\mathrm{Grenz}}=4.47 \mathrm{~nm}$ und $4.15 \mathrm{~nm}$ mit 8 Parametern konstruiert. Die Ergebnisse dieser Rechnungen zeigt Abbildung 4.9.

Die Verschiebung der Grenzfläche um eine Atomdoppellage in Richtung des amorphen Probenbereiches bedeutet mathematisch, dass die Beschreibung der atomaren Dichte für diese Lage in den Simulationen nicht mehr durch die Verteilungsfunktion, sondern durch Deltafunktionen des Kristalls erfolgt. Es ist deutlich zu erkennen, dass die Verschiebung hin $\mathrm{zu} x_{\text {Grenz }}=4.7815 \mathrm{~nm}$ mit einer Reduzierung des R-Faktors einhergeht. Die Anpassung der simulierten Abbildungen an die experimentellen Abbildungen wird also besser. Dies bedeutet, dass diese Atomdoppellage durch die verwendete Verteilungsfunktion nicht in geeigneter Weise passend beschrieben werden kann. Wird die Lage dem Kristall zugeschrieben, verbessert sich das Bildvergleichsmaß. Eine weitere Verschiebung verschlechtert die Übereinstimmung wieder, was wiederum bedeutet, dass diese Atomdoppellage besser durch die Verteilungsfunktion als durch Deltafunktionen beschrieben wird. Zusätzlich ist zu erkennen, dass sich die Beschreibung der mittleren atomaren Dichte der ersten atomaren Doppellage für die Positionen $x_{\mathrm{Grenz}}=4.47 \mathrm{~nm}$ und 

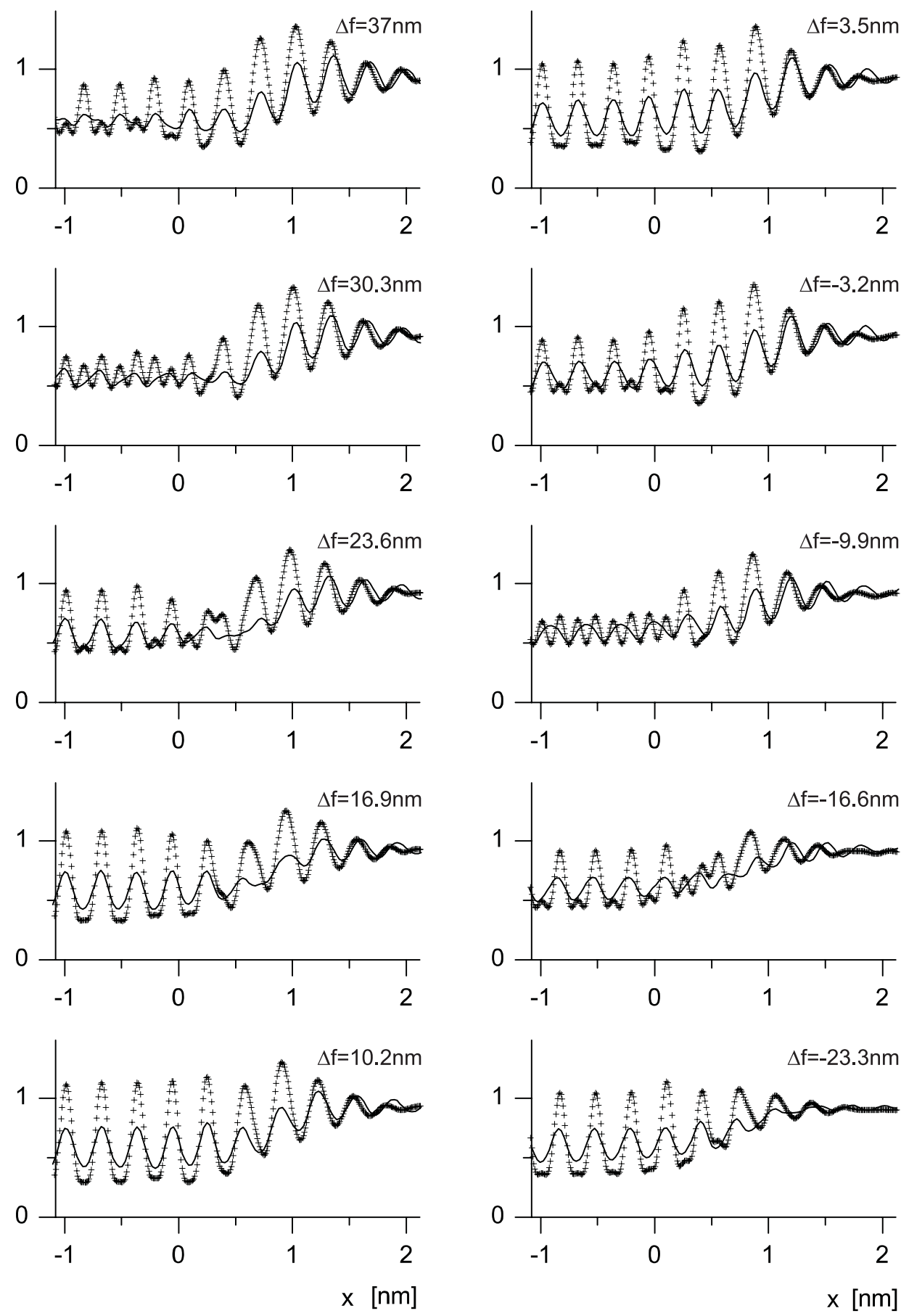

Abbildung 4.6 - Vergleich der Profile von Simulation (+) und Experiment (-) für die ersten zehn Abbildungen im Defokusbereich von $\Delta f=37, \ldots,-23.3 \mathrm{~nm}$. Es zeigt sich eine gute Übereinstimmung der Verläufe. Aufgrund des in Abschnitt 2.3.3 diskutierten Stobbs-Faktors sind die Kontraste stark unterschiedlich. Der Ursprung der $x$-Achse definiert die Grenze zwischen amorphem und kristallinem Material. 



Abbildung 4.7 - Vergleich der Profile von Simulation (+) und Experiment (-) für die zweiten zehn Abbildungen im Defokusbereich von $\Delta f=-30, \ldots,-90.3 \mathrm{~nm}$. Auch hier zeigt sich die gute Übereinstimmung der Verläufe. 

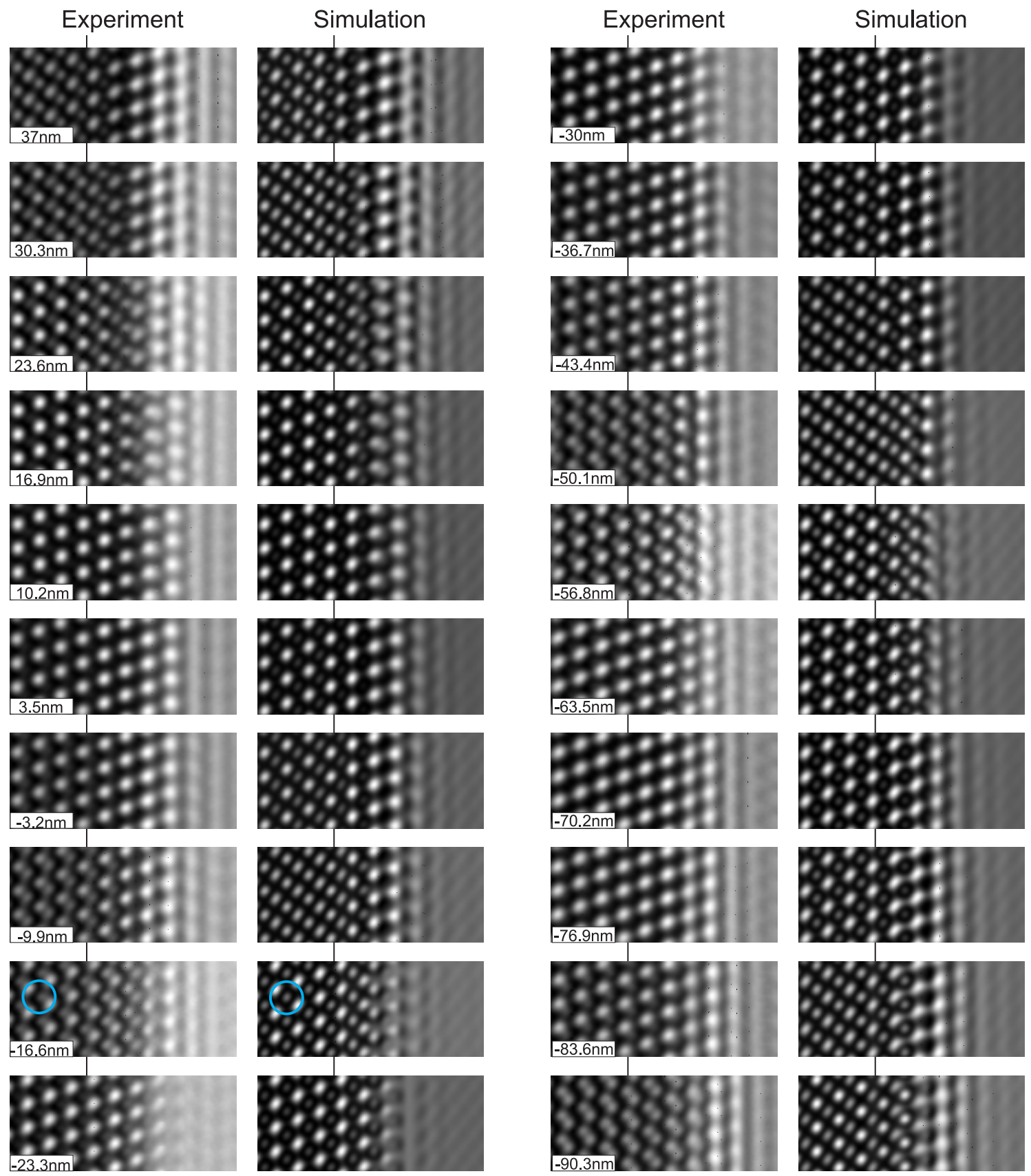

Abbildung 4.8 - Vergleich der gemittelten Grenzflächenabbildungen der gesamten a-Si/c-SiDefokusserie. Dargestellt ist jeweils die experimentelle gemittelte Abbildung (links) und die simulierte gemittelte Abbildung (rechts). Die Position des vertikalen Strichs zeigt die Position der berechneten Grenzfläche. In den experimentellen gemittelten Abbildungen ist z. T. eine Asymmetrie erkennbar (z.B. $\Delta f=-16.6 \mathrm{~nm}$, blauer Kreis), die auf eine leichte Probenverbiegung hindeutet. Dies wird in den Simulationen nicht berücksichtigt. 
(a)


(b)

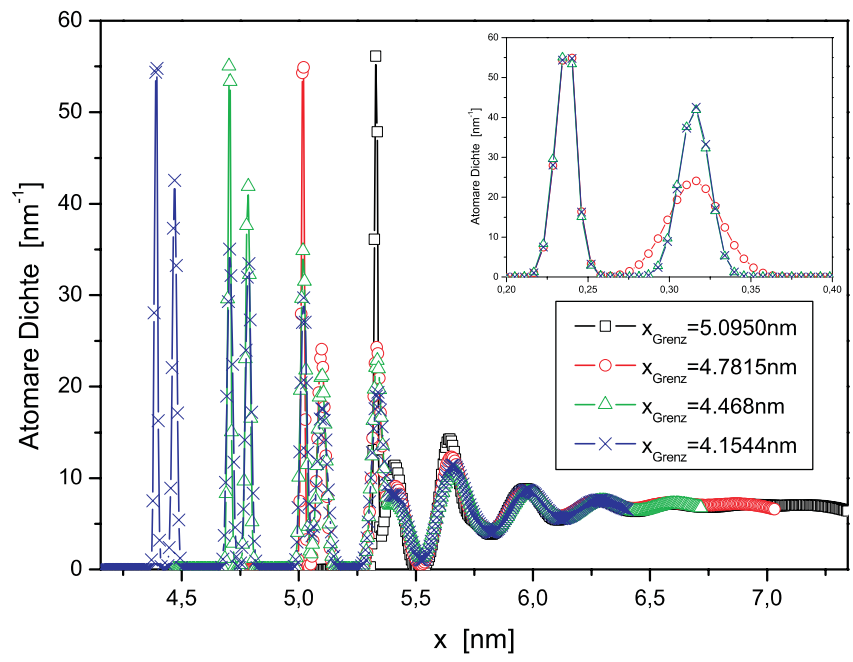

Abbildung 4.9 - Untersuchung zur Festlegung der Grenzflächenposition. (a) Die zweidimensionalen Verteilungsfunktionen für die Positionen $x_{G r e n z}=5.1 \mathrm{~nm}$ (oben, schwarz), $x_{G r e n z}=4.78 \mathrm{~nm}$ (rot), $x_{\text {Grenz }}=4.47 \mathrm{~nm}$ (grün) und $x_{\text {Grenz }}=4.15 \mathrm{~nm}$ (unten, blau). Daneben ist jeweils der R-Faktor angezeigt. Die beste Übereinstimmung wurde bei der Position $x_{\text {Grenz }}=4.78 \mathrm{~nm}$ erreicht. (b) Die eindimensionalen Profile der atomaren Dichte wurden übereinander dargestellt. Es ist gut zu erkennen, dass die erste atomare Doppellage für die beiden Verschiebungen $x_{\text {Grenz }}=4.47 \mathrm{~nm}$ und $4.15 \mathrm{~nm}$ gleich beschrieben wird. Unterschiede zu dieser Ordnung erscheinen erst für die Position bei $x_{\text {Grenz }}=4.78 \mathrm{~nm}$. Dieses Verhalten wird durch den eingelassenen Plot nochmals verdeutlich, in dem für alle drei Positionen die Verteilung der atomaren Dichte für die erste atomare Doppellage übereinander dargestellt ist. 


\begin{tabular}{cccccccc}
\hline \hline$\varphi$ & $\sigma_{\theta 1}$ & $\sigma_{\theta 2}$ & $\sigma_{\theta 3}$ & $\sigma_{\theta 4}$ & $\alpha_{1}$ & $\alpha_{2}$ & R-Faktor \\
\hline $70.5^{\circ}$ & $1.9^{\circ}$ & $3.9^{\circ}$ & $3.8^{\circ}$ & $10.7^{\circ}$ & $3.3^{\circ}$ & $6.2^{\circ}$ & 233 \\
\hline \hline
\end{tabular}

Tabelle 4.3 - Zusammenfassung der Ergebnisse der iterativen Bildanpassung für die a-Si/c-Si-Probe

$x_{\text {Grenz }}=4.15 \mathrm{~nm}$ nicht ändert (s. Abb. 4.9(b)). Diese Ergebnisse belegen, dass für diese beiden Positionen keine strukturellen Unterschiede in der jeweils ersten atomaren Doppellage existieren, was wiederum für kristalline Ordnung spricht. Erst ab der Position $x_{\text {Grenz }}=4.78 \mathrm{~nm}$ existiert ein deutlicher Unterschied in der Beschreibung der mittleren atomaren Dichte dieser Doppellage. Es muss somit gefolgert werden, dass es ab dieser Position zu Abweichungen von der kristallinen Ordnung kommt. Die Position der Grenze in der $9.4 \mathrm{~nm}$ breiten Superzelle wird dementsprechend bei $4.78 \mathrm{~nm}$ festgelegt ${ }^{\dagger}$. Der beste Parametersatz für die zweidimensionale Verteilungsfunktion an dieser Position ist in Tabelle 4.3 zusammengefasst.

Der Winkel $\varphi$ zwischen der [111]-Richtung und der Verbindungslinie zwischen den Schwerpunkten der ersten und zweiten amorphen Atomlage kann als die mittlere Richtung der Si-Si-Bindungen interpretiert werden (s. auch Abb. 4.10). Er entspricht dem Winkel des idealen Diamantgitters in dieser Projektion und beträgt $\varphi=70.5^{\circ}$. Die Standardabweichung der Verteilung der Abweichungen von der mittleren Bindungsrichtung ist in der ersten amorphen Lage mit $\sigma_{\theta 1}=1.9^{\circ}$ sehr klein. Abbildung 4.10 fasst die Ergebnisse noch einmal graphisch zusammen. Auf der linken Seite ist die Position des Grenzübergangs im analysierten Ausschnitt einer experimentellen Abbildung mit einem Pfeil gekennzeichnet, rechts ist die angepasste zweidimensionale Verteilungsfunktion $\rho(x, y)$ dargestellt. Es ist gut zu erkennen, wie durch die Bindung an die kristallperiodisch angeordneten Atome eine kristalline Ordnung in das amorphe Material induziert wird, welche von Atomlage zu Atomlage abnimmt. Fern der Grenzfläche ergibt sich schließlich eine homogene Verteilung.

Um die Ausdehnung des vom Kristall beeinflussten Übergangsbereiches mit modifizierter amorpher Struktur zu bestimmen, wurde analog zu der Vorgehensweise von BorgardT ET AL. ${ }^{[13]}$ ab einem bestimmten Punkt $x_{h}$ in der zweidimensionalen Verteilungsfunktion anstelle der vorhandenen modulierten eine homogene Verteilung angesetzt. Mittels dieser „neuen“ Verteilungsfunktion wurden wiederum gemittelte Abbildungen simuliert und diese mit den experimentellen Abbildungen anhand des R-Faktors verglichen. Ab einer Entfernung von $x_{h}=1.4 \mathrm{~nm}$ ergab sich eine Veränderung von lediglich $1 \%$ im R-Faktor im Vergleich zu der ursprünglich verwendeten Verteilungsfunktion, so dass dieser Wert analog zu BorGARDT ET AL. ${ }^{[13]}$ als Ende des Übergangsbereiches definiert wurde. Er entspricht der Weite von vier Silizium-Doppellagen.

${ }^{\dagger}$ Zur besseren Sichtbarkeit wurde in den Profilabbildungen 4.6 und 4.7 diese Grenzflächenposition auf der $x$-Achse als Ursprung gewählt. 


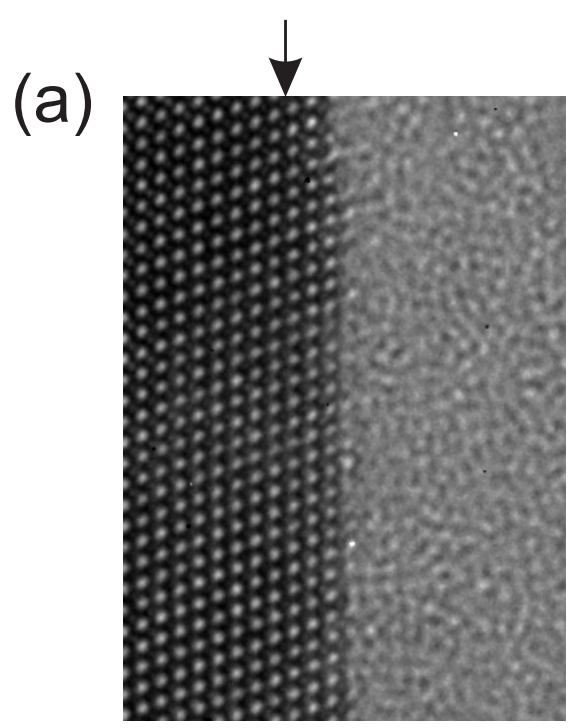

(b)



Abbildung 4.10 - Graphische Darstellung der Ergebnisse der iterativen Bildanpassung für die a$\mathrm{Si} / \mathrm{c}$-Si-Probe. (a) Position der Grenze zwischen kristallinem und amorphem Silizium. Der Pfeil zeigt die Position der letzten kristallinen Atomreihe. (b) Angepasste zweidimensionale Verteilungsfunktion $\rho(x, y)$. Der Winkel zwischen der [111]-Richtung und der Verbindungslinie zwischen den Schwerpunkten der ersten und zweiten amorphen Atomlage beträgt wie im idealen Diamantgitter $\varphi=70.5^{\circ}$.

\subsubsection{3 a-Ge/c-Si-Probe}

Das Materialsystem amorphes Germanium auf kristallinem Silizium ist bereits intensiv von Borgardt ET AL. ${ }^{[2,13]}$ und PlikAT ${ }^{[24,25]}$ behandelt worden. Dabei wurde $\rho(x, y)$ mit zwei Parametern konstruiert. Um in dieser Arbeit einen direkten quantitativen Vergleich zwischen der Untersuchung der a-Si/c-Si- und a-Ge/c-Si-Probe zu ermöglichen, musste die Defokusserie der a-Ge/c-Si-Probe noch einmal analysiert und die zweidimensionale Verteilungsfunktion mit sechs Parametern konstruiert werden.

Analog zu den Ergebnissen im letzten Abschnitt zeigen die Abbildungen 4.11 und 4.12 die Profilvergleiche nach der Anpassung und die Abbildung 4.13 den Vergleich der gemittelten experimentellen und simulierten Abbildungen.

Wie bei der a-Si/c-Si-Probe ist auch hier die sehr gute Übereinstimmung zwischen den Intensitätsverläufen in den experimentellen und simulierten gemittelten Grenzflächenabbildungen über die gesamte Defokusserie hervorzuheben. Tabelle 4.4 fasst die angepassten Parameter für die Konstruktion von $\rho(x, y)$ zusammen, und Abbildung 4.14 zeigt die resultierende zweidimensionale Verteilungsfunktion sowie die Position der Grenzfläche in einer experimentellen Abbildung.

Analog zu der Interpretation der Verteilungsfunktion des amorphen Siliziums der a$\mathrm{Si} / \mathrm{c}-\mathrm{Si}$-Probe wird die Verbindungslinie zwischen den Schwerpunkten von $\rho(x, y)$ in der ersten und zweiten atomaren Lage des amorphen Germaniums als die mittlere Richtung der Ge-Ge-Bindungen interpretiert. Diese Richtung schließst mit der Grenzflä- 

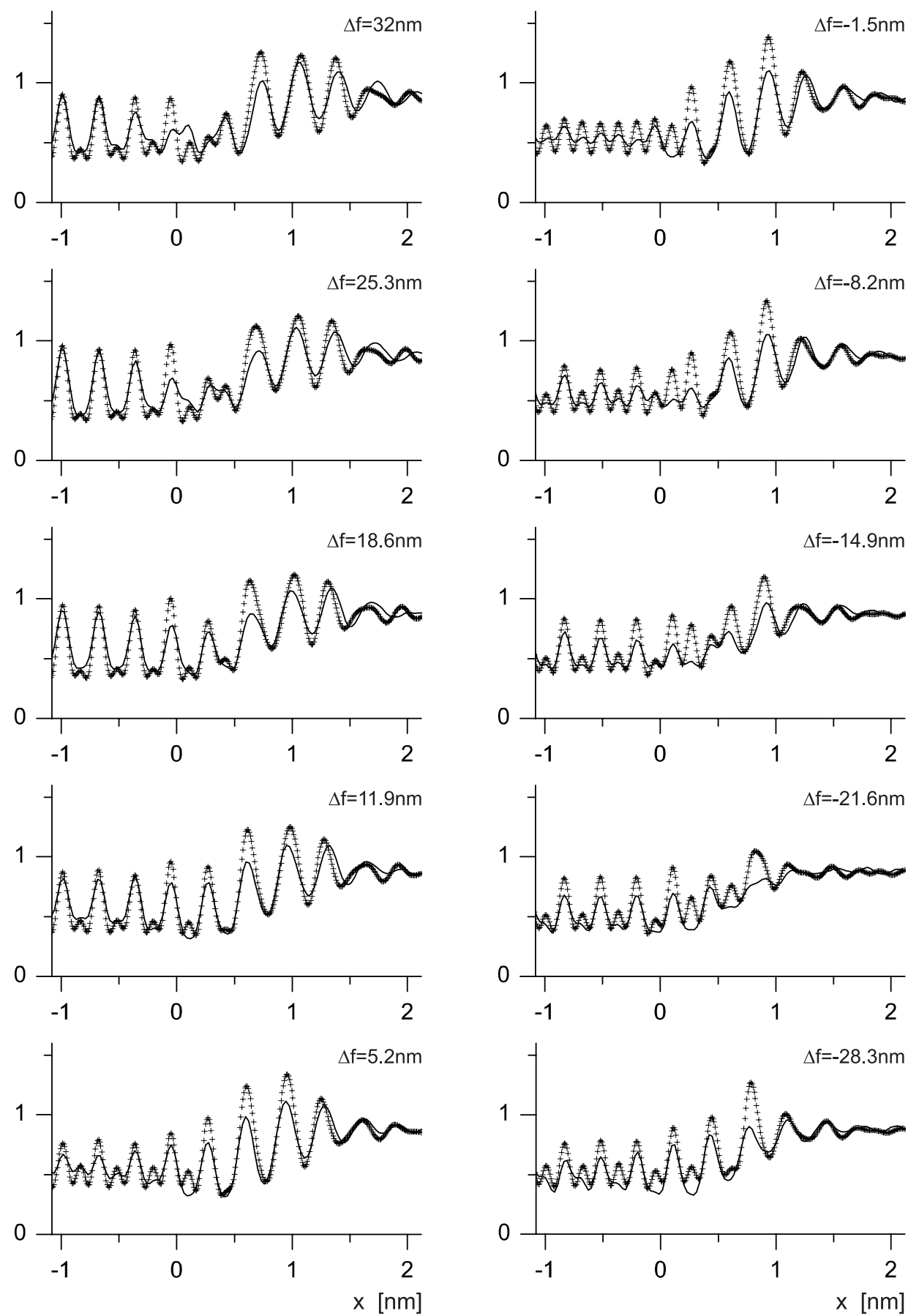

Abbildung 4.11 - Vergleich der Profile von Simulation (+) und Experiment (-) für die ersten zehn Abbildungen im Defokusbereich von $\Delta f=32, \ldots,-28.3 \mathrm{~nm}$. Es zeigt sich eine gute Übereinstimmung der Verläufe. Aufgrund des in Abschnitt 2.3.3 diskutierten Stobbs-Faktors sind die Kontraste stark unterschiedlich. Der Ursprung der $x$-Achse definiert die Grenze zwischen amorphem und kristallinem Material. 

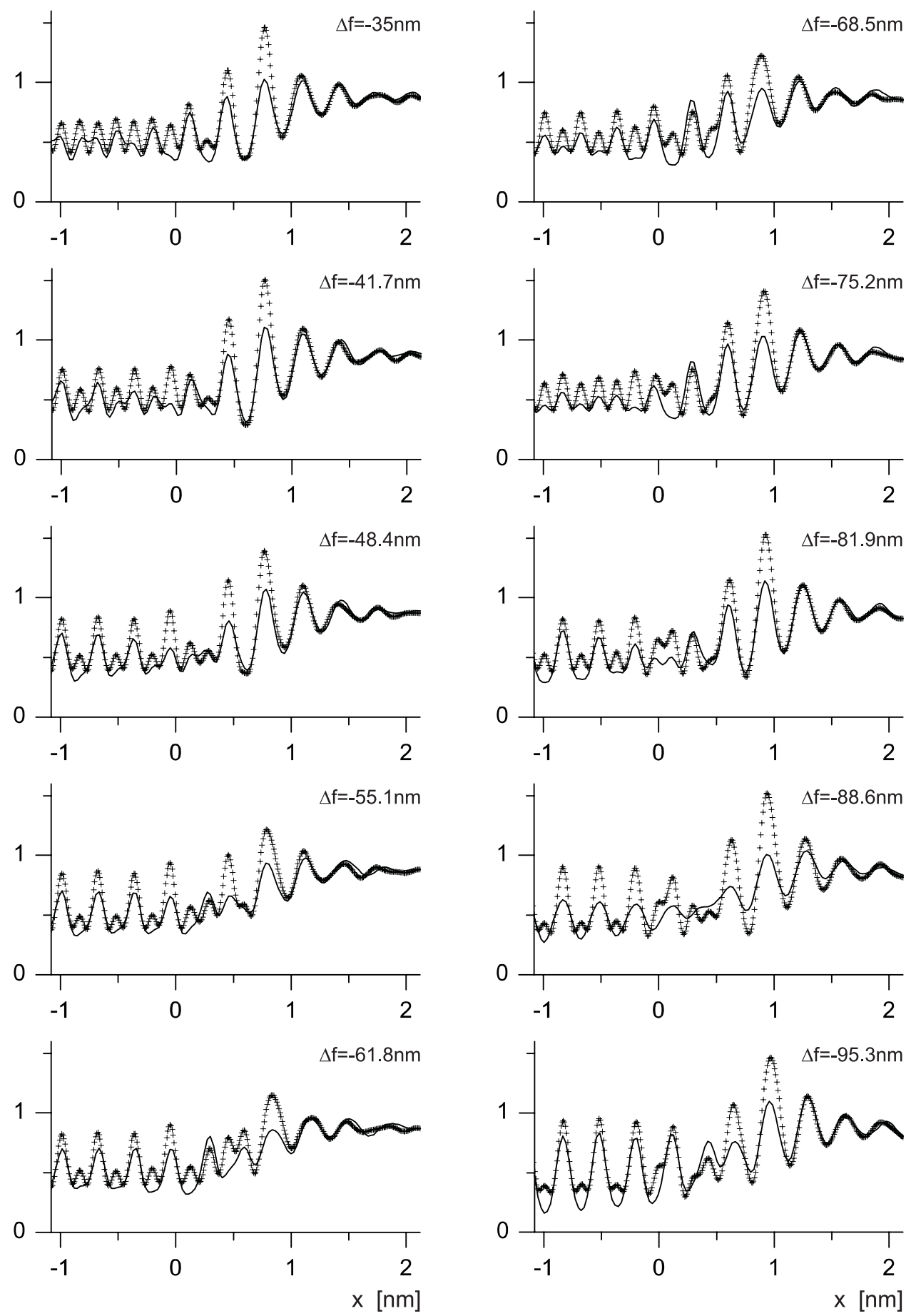

Abbildung 4.12 - Vergleich der Profile von Simulation (+) und Experiment (-) für die zweiten zehn Abbildungen im Defokusbereich von $\Delta f=-35, \ldots,-95.3 \mathrm{~nm}$. Auch hier zeigt sich eine gute Übereinstimmung der Verläufe. 
Experiment
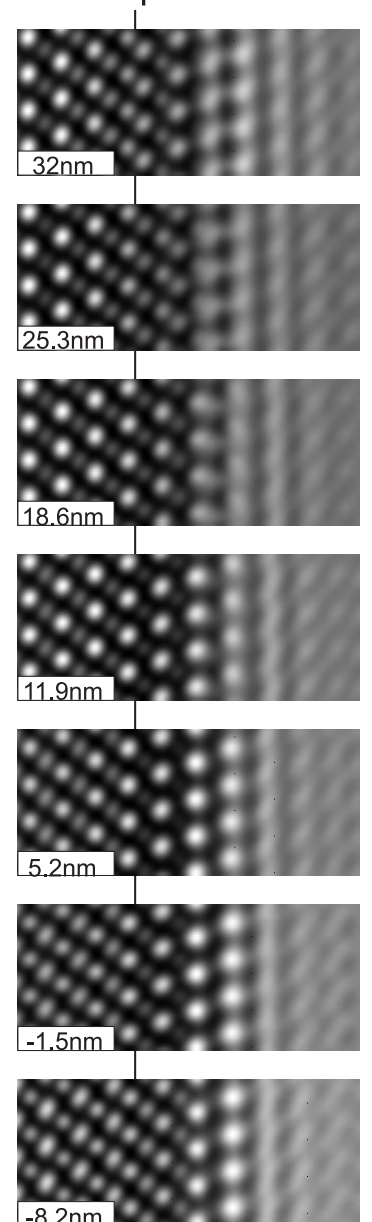

$-8.2 n m$

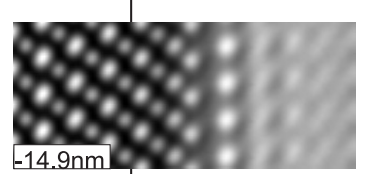

$-14.9 \mathrm{~nm}$

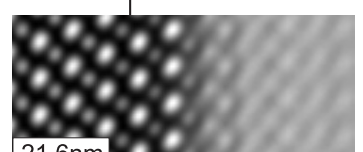

$-216 \mathrm{~nm}=0$

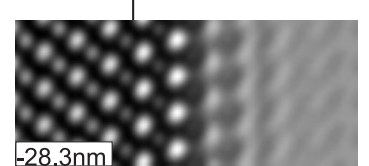

Simulation
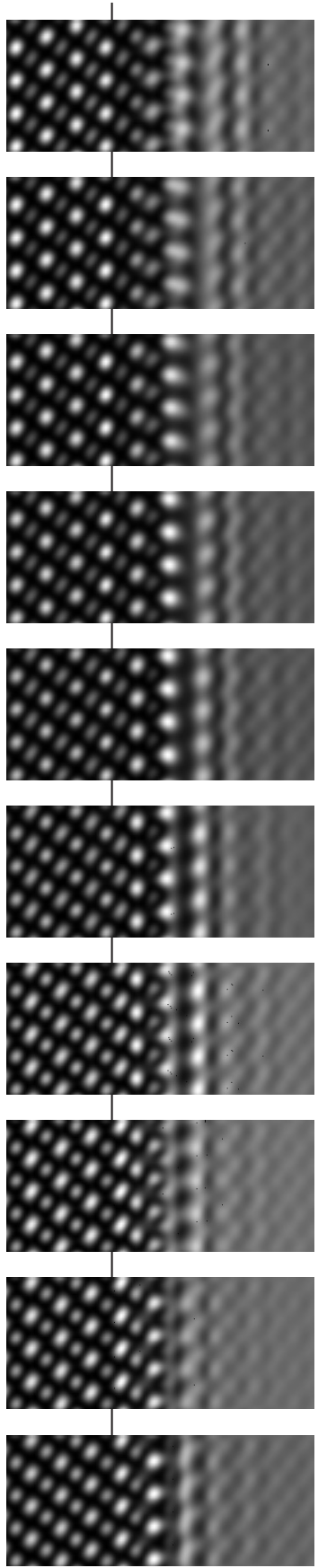

Experiment
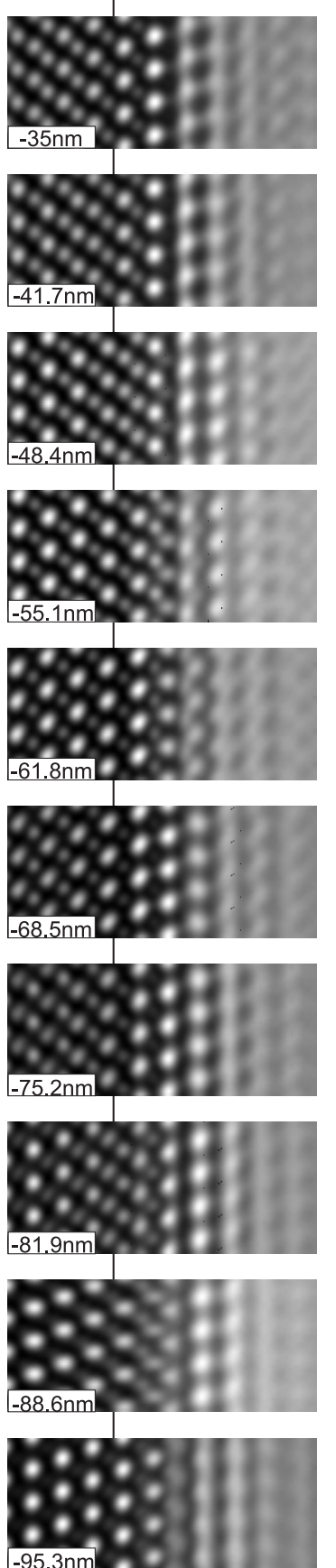

Simulation
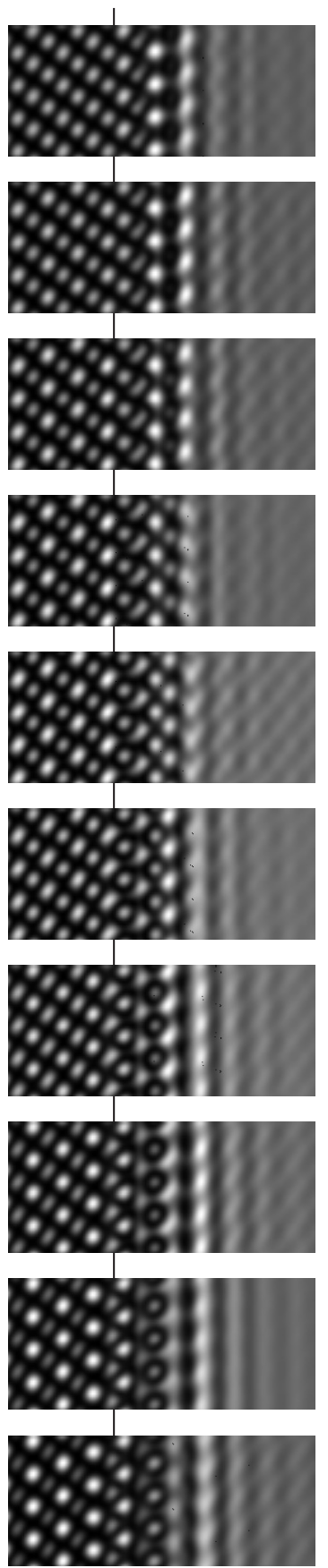

Abbildung 4.13 - Vergleich der gemittelten Grenzflächenabbildungen der gesamten a-Ge/c-SiDefokusserie. Dargestellt ist jeweils die experimentelle gemittelte Abbildung (links) und die simulierte gemittelte Abbildung (rechts). Die Position des vertikalen Strichs zeigt die Position der berechneten Grenzfläche. 


\begin{tabular}{cccccccc}
\hline \hline$\varphi$ & $\sigma_{\theta 1}$ & $\sigma_{\theta 2}$ & $\sigma_{\theta 3}$ & $\sigma_{\theta 4}$ & $\alpha_{1}$ & $\alpha_{2}$ & R-Faktor \\
\hline $65^{\circ}$ & $11.3^{\circ}$ & $6.0^{\circ}$ & $10.3^{\circ}$ & $16.2^{\circ}$ & $10.9^{\circ}$ & $3.0^{\circ}$ & 168 \\
\hline \hline
\end{tabular}

Tabelle 4.4 - Zusammenfassung der Ergebnisse der iterativen Bildanpassung für die a-Ge/c-Si-Probe

(a)



(b)



Abbildung 4.14 - Graphische Darstellung der Ergebnisse der iterativen Bildanpassung für die aGe/c-Si-Probe. (a) Position der Grenze zwischen kristallinem und amorphem Silizium. Der Pfeil zeigt die Position der letzten kristallinen Atomreihe. (b) Angepasste zweidimensionale Verteilungsfunktion $\rho(x, y)$. Der Winkel zwischen der [111]-Richtung und der Verbindungslinie zwischen den Schwerpunkten der ersten und zweiten amorphen Atomlage beträgt $\varphi=65^{\circ}$.

chennormalen den Winkel $\varphi=65^{\circ}$ ein, während er für das ungestörte Diamantgitter $\varphi=70.5^{\circ}$ beträgt. Des Weiteren ergibt sich als Standardabweichung für die Verteilung der Abweichungen von der mittleren Bindungsrichtung in der ersten amorphen Lage mit $\sigma_{\theta 1}=11.3^{\circ}$ ein etwas größerer Wert als für reines amorphes Germanium $\sigma_{\theta 1}=9.7^{\circ}[26]$. BORGARDT ET AL. ${ }^{[13]}$ interpretieren diese Ergebnisse als eine elastische Verzerrung des amorphen Germaniums im Übergangsbereich, welche durch die Volumen-Fehlpassung zwischen den Materialien hervorgerufen und durch den mittleren Bindungswinkel relaxiert wird.

Die Weite des Grenzübergangs wurde von BorgardT ET AL. anhand des im vorigen Abschnitt beschriebenen Verfahrens bereits auf etwa $1.4 \mathrm{~nm}$ abgeschätzt ${ }^{[13]}$. Die Verwendung von 6 Parametern für die Konstruktion der Verteilungsfunktion hat auf diese Weite keinen Einfluß (s. auch Abb. 4.5(a) auf Seite 59). 
(a)

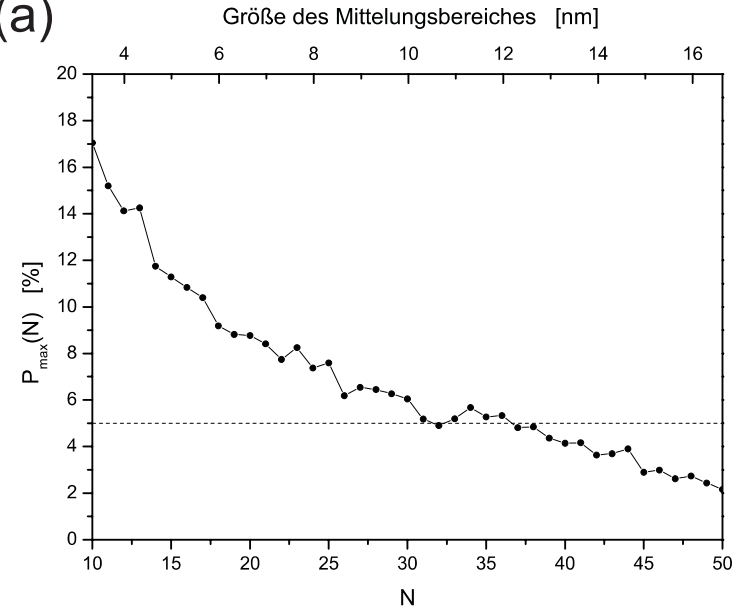

(b)

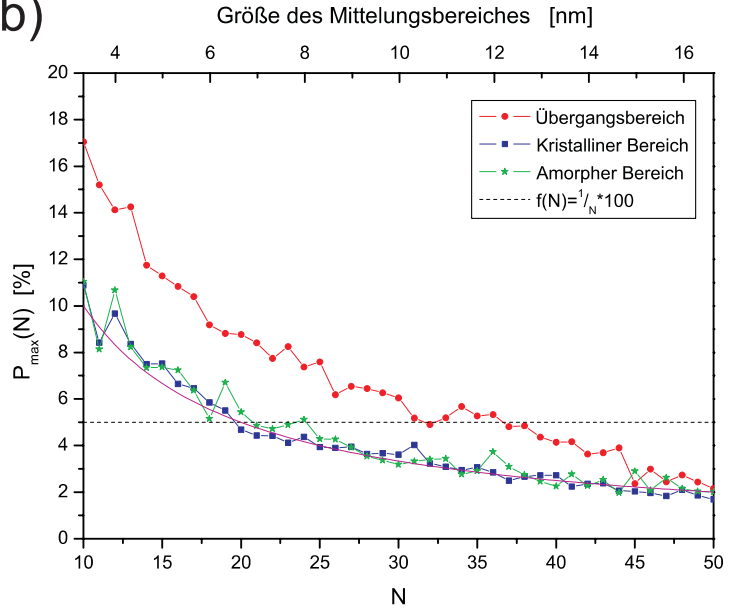

Abbildung 4.15 - Maximale relative Intensitätsvariation $P_{\max }$ aufgetragen gegen die Anzahl der Streifen $N$ innerhalb des Mittelungsbereiches (untere Achse) und die entsprechende Größe des Bereiches $A=N d$ (obere Achse). Die horizontale gepunktete Linie repräsentiert jeweils die 5\%-Grenze. (a) Das Ergebnis der Rechnung für den gesamten Anpassungsbereich. (b) Die Ergebnisse individuell für den kristallinen, den amorphen und den Übergangsbereich. Es zeigt sich, dass für $N \gtrsim 30$ die relativen Intensitätsvariationen unterhalb der Signifikanzgrenze von 5\% liegen, das Kriterium somit erfüllt ist. Weitere Erläuterungen im Text.

\subsection{Laterale Homogenität der zweidimensionalen Verteilungsfunktion}

In diesem Abschnitt soll der strukturelle Übergang entlang der Grenzfläche im Hinblick auf die Homogenität der zweidimensionalen Verteilungsfunktion entsprechend der in Abschnitt 3.3 vorgestellten Methode untersucht werden. Abschnitt 4.2.1 befasst sich dabei mit dem Übergang zwischen amorphem Germanium und kristallinem Silizium, und Abschnitt 4.2.2 behandelt den Fall des amorphen Siliziums auf kristallinem Silizium.

\subsection{1 a-Ge/c-Si-Probe}

Entsprechend dem in Abschnitt 3.3 beschriebenen Verfahren wurde die maximale relative Intensitätsvariation für verschiedene Ausdehnungen des Mittelungsbereiches berechnet. Abbildung 4.15(a) zeigt das Ergebnis für die Defokusserie der Germanium-Probe für Ausdehnungen von $N=5, \ldots, 50$ Streifen.

Die relative Intensitätsvariation zwischen zwei gemittelten Abbildungen, wie in Gleichung 3.26 definiert, wurde für denselben Bereich berechnet wie der R-Faktor bei dem Bildvergleich zwischen Simulation und Experiment, d.h. für $-0.48 \leq x \leq 2.02 \mathrm{~nm}$ und $0 \leq y \leq 0.665 \mathrm{~nm}$ (siehe Abschnitt 4.1.2).

Zur Abschätzung nicht-signifikanter Intensitätsvariationen wurde als oberer Grenzwert 
der in der Statistik häufig gewählte Wert von $5 \%$ benutzt. Der sich daraus ergebende minimale Mittelungsbereich von $N \approx 30$ Streifen ist konsistent mit Testrechnungen von BorgardT ET AL., die gezeigt haben, dass bei einer Ausdehnung des Mittelungsbereiches von 28 Streifen durch die Verwendung der APP-Näherung die Genauigkeit der Modellparameter auf $\leq 5 \%$ abgeschätzt werden kann ${ }^{[13]}$. Deswegen wurde für die folgenden Anwendungen die Größe des Mittelungsbereiches auf dreißig Streifen, i.e. $\approx 10 \mathrm{~nm}$, festgelegt.

In Teil (b) der Abbildung 4.15 sind die Rechnungen für die drei Bereiche kristallin, Übergang und amorph gezeigt. Dabei bezeichnet kristallin den Bereich von $-0.48 \leq x \leq$ 0nm, der Übergangsbereich ist durch $0<x \leq 1.4 \mathrm{~nm}$ gegeben und der amorphe Bereich schließlich durch $1.4<x \leq 2.02 \mathrm{~nm}$. In Abbildung 3.8 (s. Seite 44) sind die Grenzen der drei Bereiche an den gemittelten Abbildungen durch vertikale Striche angedeutet.

In Abschnitt 3.3.1 wurde bereits diskutiert, welche Unterschiede für die relativen Intensitätsvariationen der unterschiedlichen Bereiche der Grenzfläche zu erwarten sind. Dort wurde postuliert, dass die größte relative Intensitätsvariation aufgrund struktureller Inhomogenitäten entlang der Grenzfläche im Übergangsbereich zwischen kristallinem und amorphem Material zu erwarten ist, während sie im rein kristallinen bzw. rein amorphen Probenteil durch statistische Fluktuationen lediglich kleinere Werte annimmt. Dieses Verhalten wird anhand der Ergebnisse in Abbildung 4.15(b) verifiziert.

Die drei Kurven müssen sich mit zunehmendem $N$ einer $1 / N$ Kurve annähern, da durch die Definition der maximalen relativen Intensitätsvariation (siehe Gleichung 3.26) irgendwann die Anzahl der Streifen $N$ gegenüber der Intensitätsvariation in äquivalenten Bildpunkten dominiert. Während dies für den kristallinen und amorphen Bereich fast über den gesamten untersuchten Bereich der Fall ist, weicht der Übergangsbereich wie erwartet davon ab.

Um systematische Informationen über die Variationen in den zweidimensionalen Verteilungsfunktionen $\rho(x, y)$ entlang der Grenzfläche zu erhalten, wurde der in seiner Ausdehnung auf dreißig Streifen festgelegte Auswertungsbereich entlang der Grenze sukzessive um jeweils einen Streifen verschoben. Die Position des Bereiches wird dabei durch die Nummer des untersten Streifens $J$ bezeichnet. Der Auswertungsbereich einer solchen Position wird im Folgenden als „Serie. $J “$ bezeichnet. Jede neue Position des Auswertungsbereiches ergibt eine neue Serie gemittelter Grenzflächenabbildungen und somit nach der iterativen Bildanpassung eine neue zweidimensionale Verteilungsfunktion $\rho^{(J)}(x, y)$.

In Abschnitt 4.1.1 wurde die Grenzfläche bereits in siebzig Streifen unterteilt (0 bis 69, siehe Abbildung 4.2 auf Seite 54), was bei einer Größe des Mittelungsbereiches von 30 Streifen zu 41 Serien führt. Für jede fünfte Serie wurde die Bootstrap-Analyse mit jeweils 50 synthetischen Datensätzen durchgeführt.

Die Ergebnisse sollen exemplarisch für drei Bereiche an der Grenzfläche dargestellt und miteinander verglichen werden. Dabei handelt es sich um die Serie.0 (unterer Bereich, Streifen 0 bis 29), Serie.20 (mittlerer Bereich, Streifen 20 bis 49) und Serie.30 (oberer Bereich, Streifen 30 bis 59). 

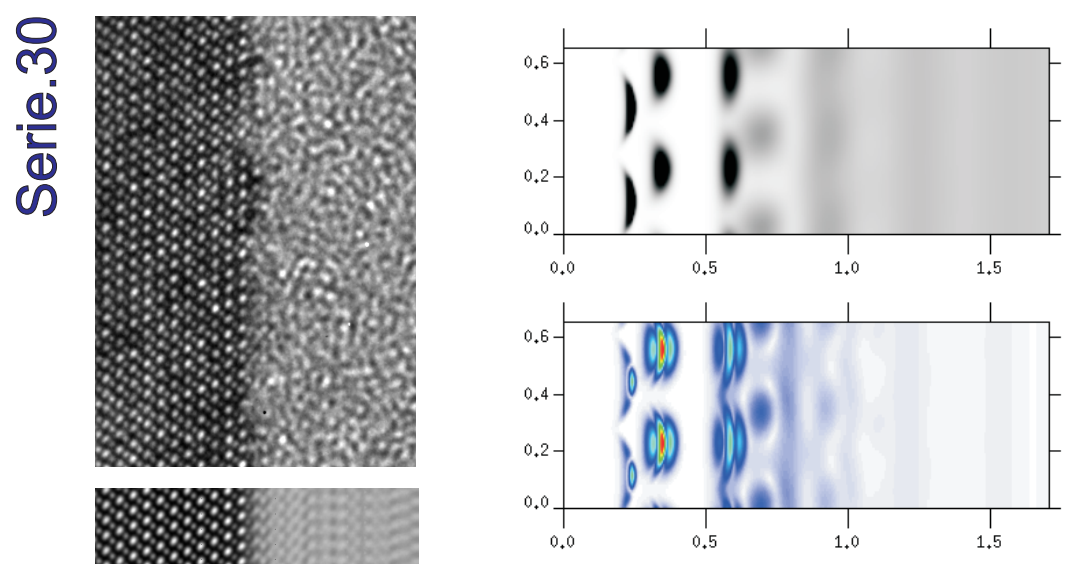

Atomare Dichte $\left[\mathrm{nm}^{-2}\right]$
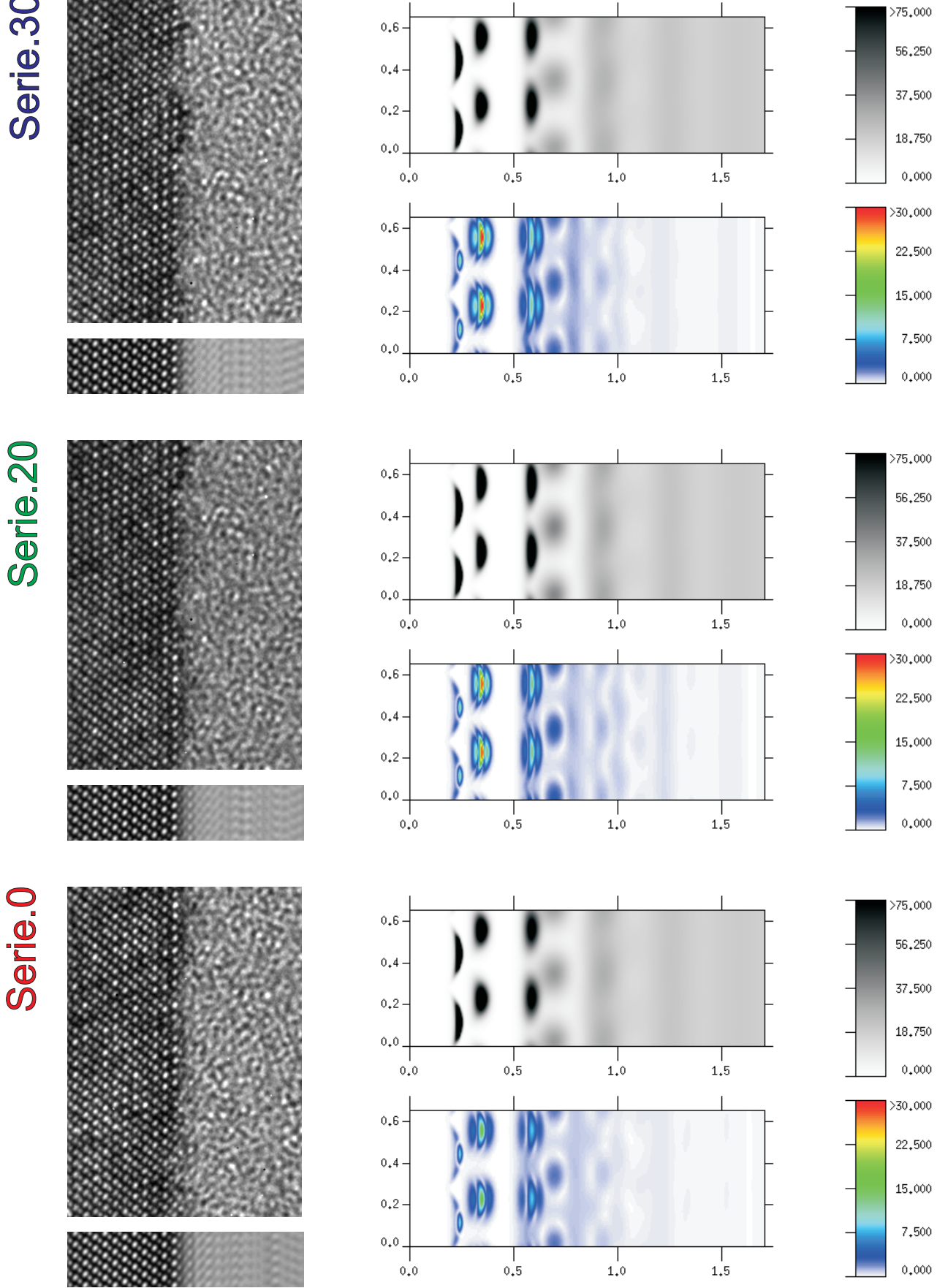

Abbildung 4.16 - Ergebnisvergleich der iterativen Bildanpassung und der Bootstrap-Analyse für die drei Serien 0, 20 und 30. Für alle Serien sind jeweils auf der linken Seite der experimentelle Bildausschnitt (oben) und die fünffach fortgesetzte gemittelte Grenzflächenabbildung (unten) gezeigt. Auf der rechten Seite ist jeweils die aus der Anpassung resultierende zweidimensionale Verteilungsfunktion (oben) und der Fehler für jeden Bildpunkt (unten) zu sehen. 
(a)
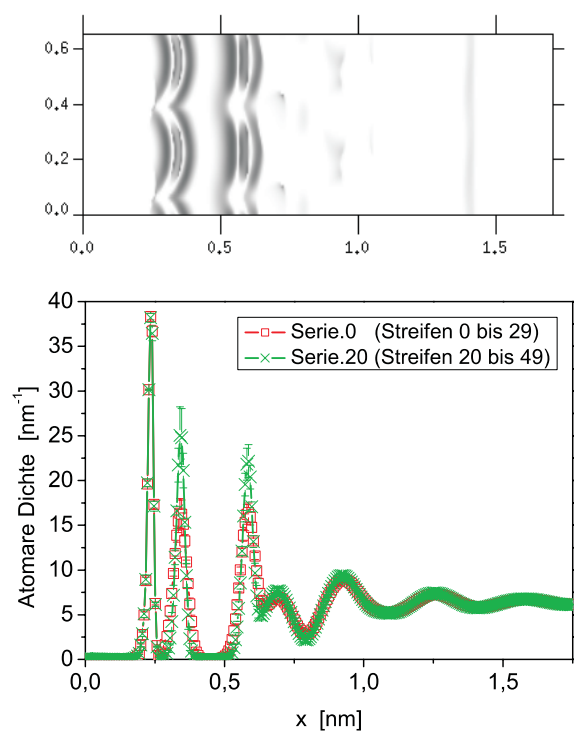

(b)

Serie. 0 - Serie. 30
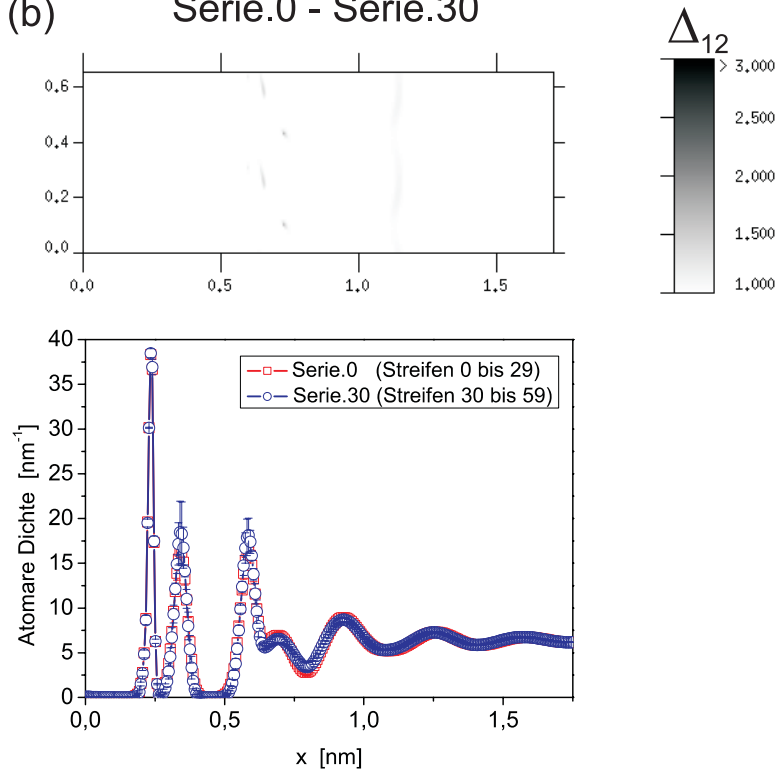

Abbildung 4.17 - Signifikante Unterschiede in den Verteilungsfunktionen zwischen den Serien 0 und 20 (a) sowie 0 und 30 (b). Jeweils oben ist die Verteilung des Signifikanzwertes $\Delta_{12}$ dargestellt, wie er in Gleichung 4.1 definiert wurde. Der Vergleich der Profile mit ihren Fehlerbalken ist unten abgebildet. Es zeigt sich, dass signifikante Unterschiede $\left(\Delta_{12} \geq 1\right)$ erst ab der zweiten atomaren Lage auftreten.

Die Ergebnisse der iterativen Bildanpassung und der Fehlerrechnung zeigt Abbildung 4.16. Dabei sind für jede Serie auf der linken Seite der experimentelle Bildausschnitt (oben) und die fünffach fortgesetzte gemittelte Grenzflächenabbildung (unten) gezeigt. Auf der rechten Seite ist oben die aus der iterativen Bildanpassung resultierende zweidimensionale Verteilungsfunktion $\rho^{(J)}(x, y)$ und unten der Fehler in jedem Bildpunkt $\sigma_{\rho^{(J)}}(x, y)$ dargestellt.

Um zu entscheiden, inwieweit die Unterschiede in einem Bildpunkt signifikant sind, wurde die statistische Diskrepanz verwendet. Sie ist definiert als die Differenz zweier Messwerte und wird als signifikant eingestuft, wenn ihr Wert größer oder gleich der Summe der Fehler der beiden Messwerte ist ${ }^{[117]}$. Dies bedeutet, dass in

$$
\Delta_{12}(x, y)=\frac{\left|\rho^{(1)}(x, y)-\rho^{(2)}(x, y)\right|}{\sigma_{\rho^{(1)}}(x, y)+\sigma_{\rho^{(2)}}(x, y)}
$$

Funktionswerte von $\Delta_{12}(x, y) \geq 1$ Signifikanz anzeigen.

Den Vergleich der Serie.0 mit Serie.20 sowie der Serie.0 mit Serie.30 zeigt Abbildung 4.17, wobei im unteren Bereich die beiden Profile der atomaren Dichte mit den entsprechenden Fehlern verglichen werden. Oberhalb der Verläufe stellt eine zweidimensionale Verteilung die Signifikanz der Diskrepanz $\Delta_{12}(x, y)$ in jedem Bildpunkt zwischen zwei zweidimensionalen Verteilungsfunktionen $\rho^{(1)}(x, y)$ und $\rho^{(2)}(x, y)$ dar. 
Es ist deutlich zu erkennen, dass signifikante Unterschiede in den drei exemplarischen Verteilungsfunktionen erst ab der zweiten Atomlage auftreten, während die erste atomare Lage entsprechend dem hier verwendeten Maß unverändert bleibt.

Das Ergebnis der gesamten Untersuchung aller 41 Serien wird in Abbildung 4.18(a) anhand der Profile der atomaren Dichte gezeigt, welche zur besseren Visualisierung farbkodiert und übereinander dargestellt sind. Der unterste Streifen steht somit für Serie.0 und der oberste für Serie.40. Die Profile enthalten aber keine Aussage über die Form der Verteilung in $y$-Richtung.

Deshalb wurde für die ersten drei Atomlagen zusätzlich die Fläche bestimmt, innerhalb derer - ausgehend vom Maximum der Verteilungsfunktion für die Atomlage - sich das Atom mit $\approx 66 \%$ Wahrscheinlichkeit befindet. Abbildung 4.18(b) illustriert farbkodiert den Bereich für die drei Lagen, und (c) zeigt den Verlauf der Größe dieser Fläche für alle Serien.

Es zeigt sich das bereits in den Beispielen angedeutete Verhalten. Die Verteilung der ersten atomaren Lage ist für alle Positionen des Auswertungsbereiches entlang der Grenzfläche identisch. Dies weist auf homogene Eigenschaften dieser Atomlage auf der Skala des Bildausschnitts $(\approx 23 \mathrm{~nm})$ hin. Erst ab der zweiten atomaren Lage zeigen sich signifikante Unterschiede.

Die Weite des Übergangsbereiches jedenfalls ist davon nicht beeinflusst. Sie beträgt für alle Positionen des Auswertungsbereiches $\approx 1.4 \mathrm{~nm}$.

\subsection{2 a-Si/c-Si-Probe}

Im Gegensatz zu der Grenzfläche zwischen amorphem Germanium und kristallinem Silizium weist die Grenzfläche zwischen amorphem und kristallinem Silizium deutliche strukturelle Inhomogenitäten (kleine Inseln, Stufen) auf, wie in Abbildung 4.1 auf Seite $53 \mathrm{zu}$ sehen ist. Eine Beschreibung der grenzflächeninduzierten Ordnung im amorphen Material durch eine Abfolge von Verteilungsfunktionen entlang der Grenzfläche, wie im vorigen Abschnitt 4.2.1 durchgeführt, ist jedoch nur dann sinnvoll, wenn im Mittelungsbereich keine deutlichen strukturellen Inhomogenitäten auftreten. Dies führt sonst zu großen Varianzen in den Intensitäten der gemittelten Grenzflächenabbildungen und somit zu großen Unsicherheiten in der zweidimensionalen Verteilungsfunktion. In dem hier vorliegenden Fall müsste jedoch die Ausdehnung des Mittelungsbereiches kleiner als die minimal für eine sinnvolle statistische Beschreibung nötige Größe gewählt werden, weswegen an dieser Grenzfläche keine systematischen Untersuchungen durchgeführt werden konnten. Lediglich der in Abschnitt 4.1 untersuchte Bereich der a-Si/c-Si-Grenzfläche (Streifen 19 bis 48) zeigt über dem verwendeten Mittelungsbereich von 30 Streifen keine deutlichen strukturellen Inhomogenitäten und ermöglichte so die an dieser Position in Abschnitt 4.1 durchgeführte Analyse.

Der Einfluss der strukturellen Inhomogenitäten wird bereits bei der Berechnung der mittleren experimentellen Grenzflächenabbildungen deutlich. Zu diesem Zweck zeigt Ab- 
(a)

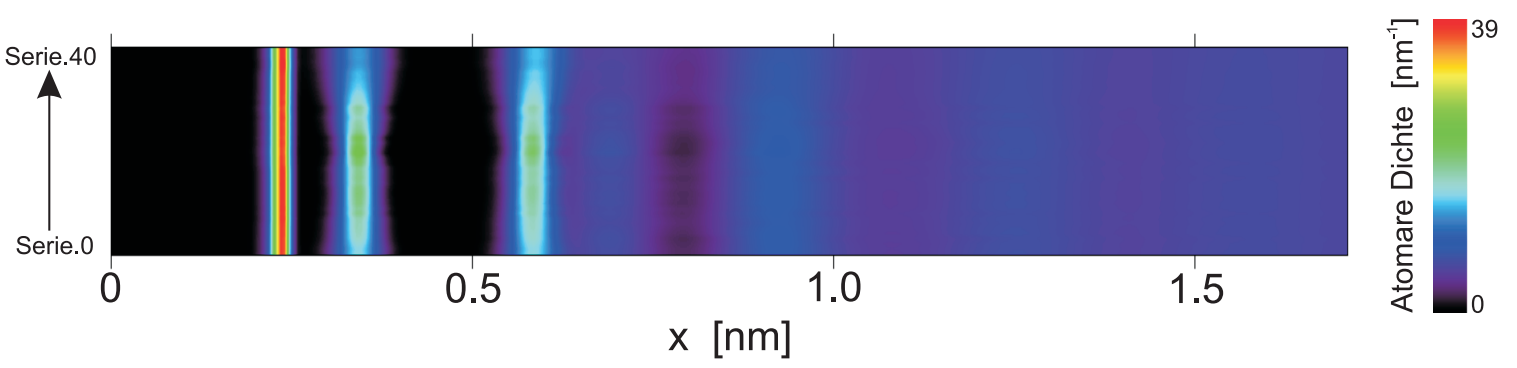

(b)

(c)
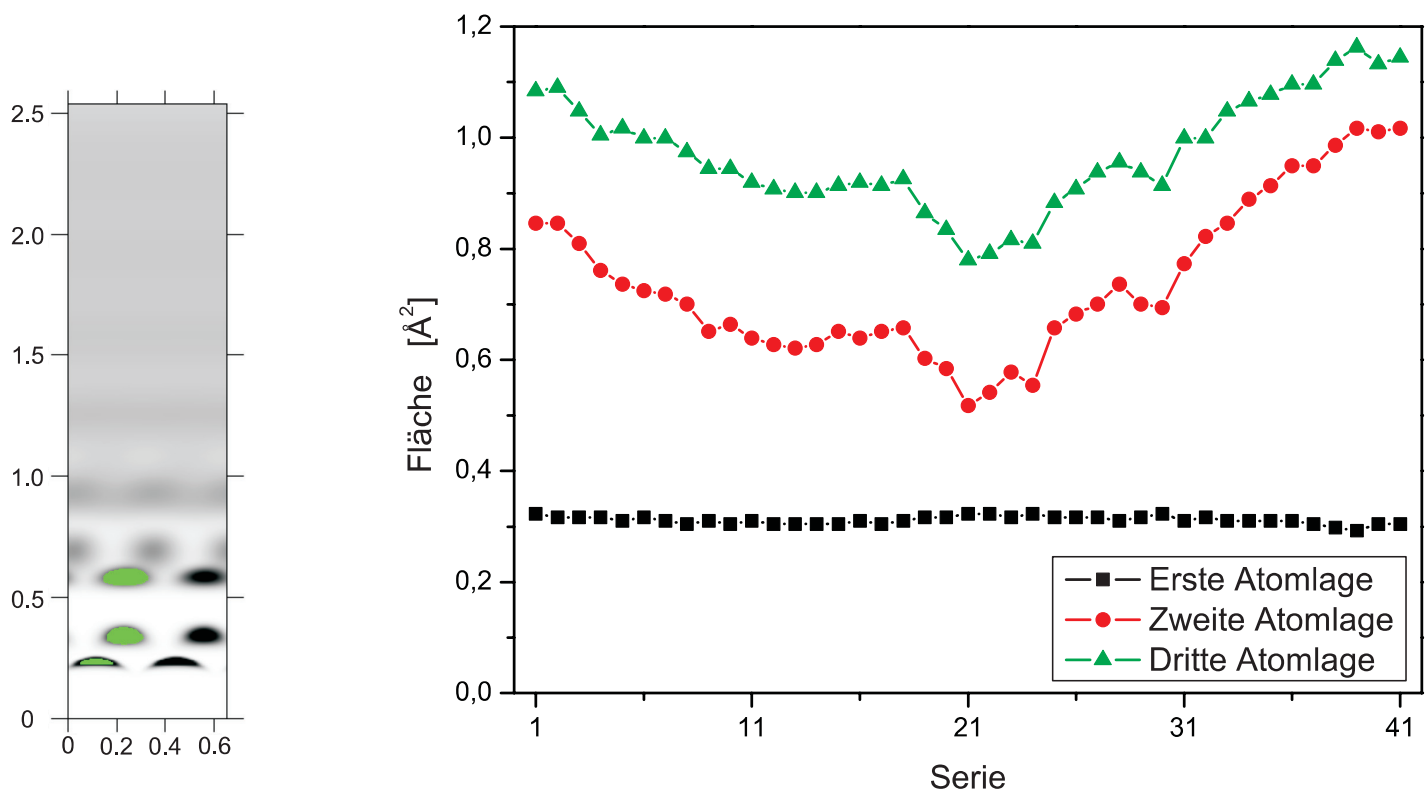

Abbildung 4.18 - (a) Abfolge der 41 Profile der systematischen Untersuchung der Grenzfläche. Die atomare Dichte wurde farbkodiert und die Profile übereinander dargestellt. (b) Für die ersten drei Atomlagen ist die Fläche, innerhalb derer sich das Atom mit $\approx 66 \%$ Wahrscheinlichkeit befindet, farblich markiert. (c) Verlauf der Größe der Fläche für die drei Atomlagen für alle Serien. Es ist deutlich zu erkennen, dass die Verteilung der ersten atomaren Lage für alle Positionen des Auswertungsbereiches keine signifikanten Unterschiede aufweist. Erst in der zweiten und dritten Lage zeigen sich Unterschiede. Die Weite des Übergangsbereiches ist von der lateralen Position des Auswertungsgebietes nicht beeinflusst. 
bildung 4.19 exemplarisch für eine beliebige Position des Mittelungsbereiches jeweils links den Mittelungsbereich und daneben das Differenzbild, welches die Differenz zwischen dem Mittelungsbereich und der $N$-fach fortgesetzten gemittelten Grenzflächenabbildung ist.

Es ist deutlich zu erkennen, dass die Ausdehnung des gewählten Mittelungsbereiches von 30 Streifen in diesem Fall zu groß ist. Im Differenzbild sind noch offensichtliche kristalline „Restintensitäten“ an der Grenzfläche zu erkennen (s. die eingekreisten Bereiche in Abb. 4.19). Im Gegensatz dazu zeigt das Differenzbild der a-Ge/c-Si-Probe diese Restintensitäten nicht. 
(a)
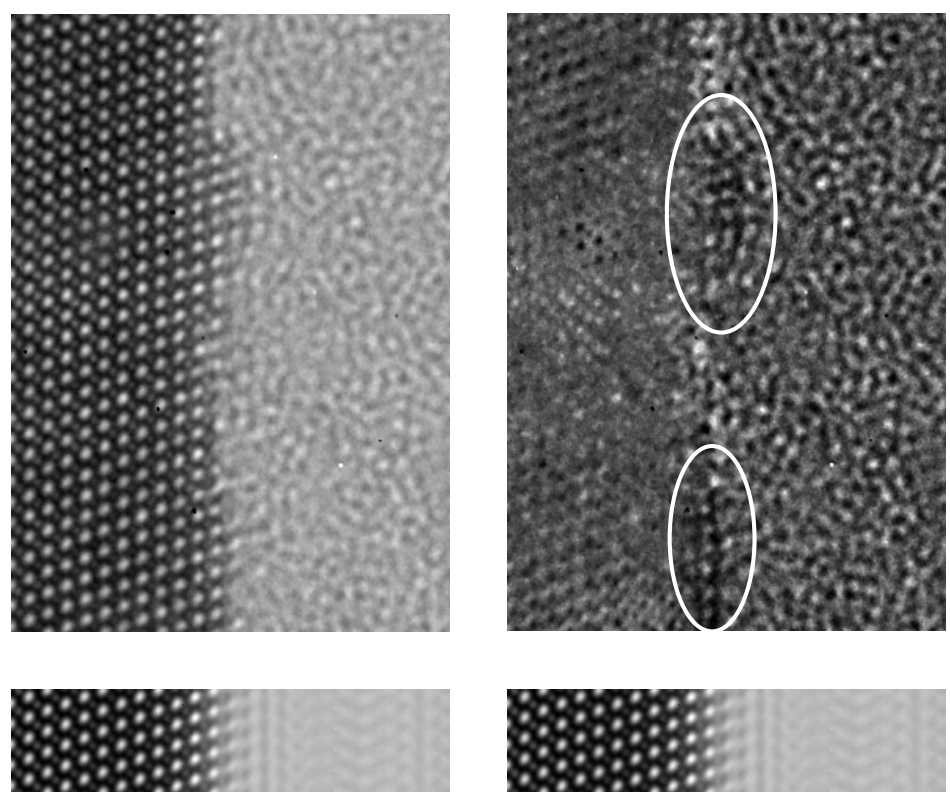

(b)
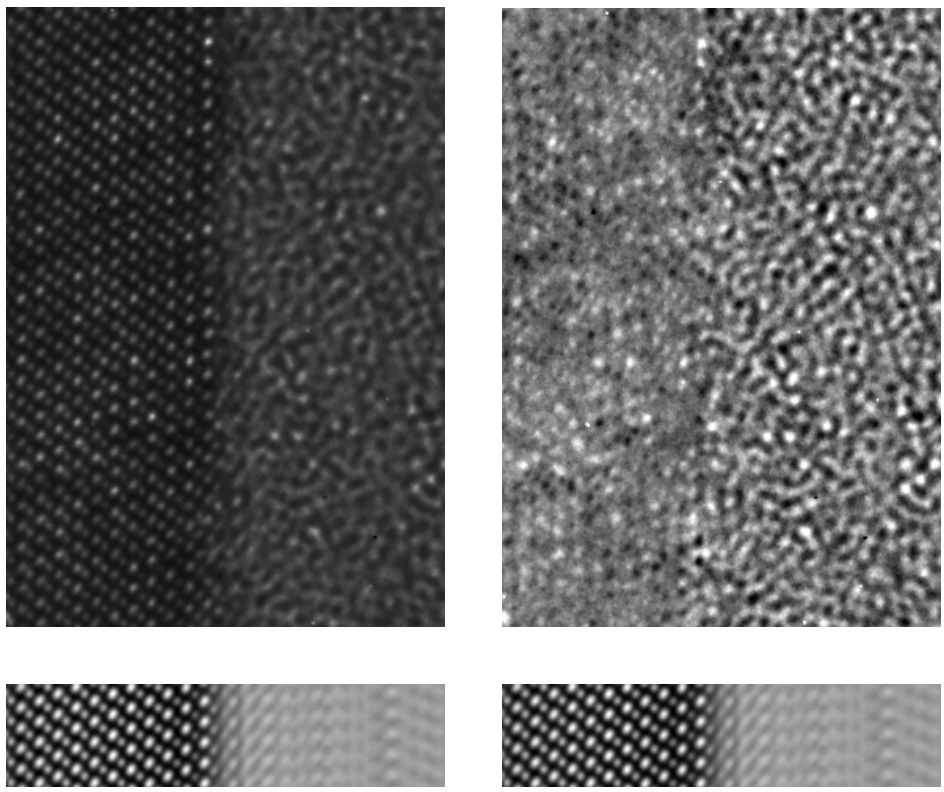

Abbildung 4.19 - Strukturelle Inhomogenitäten und ihre Auswirkungen auf die gemittelte Grenzflächenabbildung im Fall der (a) a-Si/c-Si- und (b) a-Ge/c-Si-Grenzfläche. Es sind jeweils der experimentelle Bildausschnitt (links) und das Differenzbild (rechts) dargestellt. Jeweils unter den Abbildungen ist die gemittelte Grenzflächenabbildung gezeigt. Es ist offensichtlich, dass die hier gewählte Ausdehnung des Mittelungsbereiches von 30 Streifen an dieser Position der a-Si/c-Si-Grenzfläche zu groß ist. Im Differenzbild sind deutlich kristalline „Restintensitäten“ an der Grenzfläche zu erkennen (eingekreiste Bereiche). Im Gegensatz dazu zeigt das Differenzbild der a-Ge/c-Si-Grenzfläche dieses Verhalten nicht. 


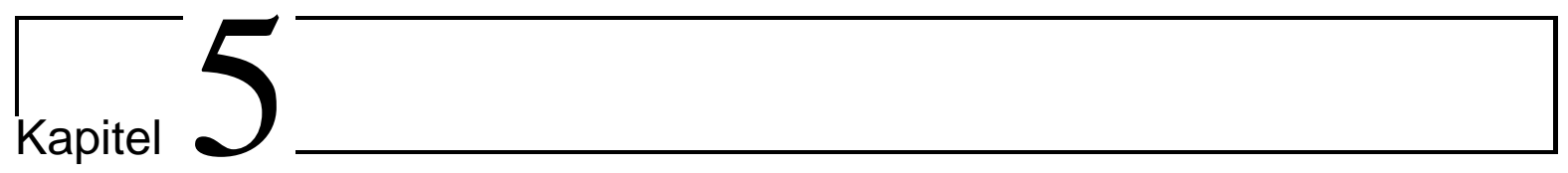

\section{Zusammenfassende Diskussion und Ausblick}

\section{Inhaltsangabe}

5.1 Zusammenfassende Diskussion . . . . . . . . . . 79

5.1.1 Die Rolle der Volumenfehlpassung . . . . . . . . . . . . . . 80

5.1.2 Untersuchung der Homogenität der zweidimensionalen Verteilungsfunktion entlang der Grenzfläche . . . . . . . . . . 85

5.2 Ausblick .................... 86

\subsection{Zusammenfassende Diskussion}

In dieser Arbeit wurden Grenzflächen zwischen kovalent gebundenen kristallinen und amorphen Materialien im Hinblick auf die induzierte Ordnung in Grenzflächennähe untersucht. Dies geschah durch den Vergleich experimenteller und simulierter elektronenmikroskopischer Grenzflächenabbildungen. Als Materialsysteme dienten zum einen die Grenzfläche zwischen amorphem Germanium und kristallinem Silizium und zum anderen die Grenzfläche zwischen amorphem Silizium und kristallinem Silizium. Des Weiteren wurden die lateralen strukturellen Unterschiede entlang der Grenzfläche zwischen amorphem Germanium und kristallinem Silizium untersucht.

Die Proben wurden im Fall der a-Ge/c-Si-Probe mittels Molekularstrahlepitaxie und bei der a-Si/c-Si-Probe durch Sputtern auf (111)-orientierten Siliziumsubstraten mit sehr geringer Fehlorientierung $\left(\approx 0.1^{\circ}\right)$ hergestellt. Eine spezielle Probenpräparation nach Suhren ET AL. ${ }^{[118]}$ stellte sicher, dass vor der Materialdeposition atomar glatte Substratoberflächen mit einer Terassenweite von $\approx 170 \mathrm{~nm}$ ausgebildet wurden. Die Grenzfläche zwischen kristallinem und amorphem Material wurde schließlich mittels hochauflösender Transmissionselektronenmikroskopie in Querschnittsgeometrie abgebildet. 
Um den Einfluss der kristallinen Ordnung auf das amorphe Material zu bestimmen, wurden die HREM-Abbildungen entlang der Grenzfläche periodisch gemittelt. Auf diese Weise konnten die Intensitätsanteile, die mit der Periode der Gitterabbildung korreliert sind, von den statistischen Intensitätsfluktuationen getrennt werden, welche für Abbildungen amorpher Materialien charakteristisch sind.

Für die Quantifizierung des Einflusses des langreichweitig geordneten kristallinen Materials auf die Struktur des amorphen Materials in Grenzflächennähe diente als Basis der iterative Vergleich von experimentellen und simulierten elektronenmikroskopischen Abbildungen ${ }^{[8,9]}$. Die strukturelle Beschreibung der Materialien für die Simulationen geschieht dabei üblicherweise im Rahmen einer Superzelle, für die die individuellen Atompositionen bekannt sein müssen. Da amorphe Materialien sinnvoll nur anhand statistischer Verteilungsfunktionen beschrieben werden können, war diese Methode nicht direkt anwendbar. Stattdessen wurde der Ansatz von BORGARDT ET AL. ${ }^{[10-13]}$ gewählt, der auf dem quantitativen Vergleich gemittelter experimenteller und simulierter Grenzflächenabbildungen beruht. Grundlage für die statistische Beschreibung der Struktur des amorphen Materials in den Simulationen ist dabei eine dreidimensionale Verteilungsfunktion $\rho_{3 \mathrm{D}}$, welche die Wahrscheinlichkeit beschreibt, ein Atom im amorphen Material zu finden, wenn $\vec{r}=0$ die Position eines Atoms im Kristallinen ist. BorgARDT ET AL. haben gezeigt, dass innerhalb der so genannten APP-Näherung gemittelte Grenzflächenabbildungen auf der Basis konventioneller Multislice-Simulationen unter Verwendung der zweidimensionalen Projektion von $\rho_{3 \mathrm{D}}$ direkt $^{[11]}$ und hinreichend genau ${ }^{[13]}$ simuliert werden können.

Um den Einfluss der defokusabhängigen Delokalisierung von dem der grenzflächeninduzierten Ordnung auf die Strukturbestimmung zu trennen, wurden Defokusserien untersucht, d.h. dass 20 gemittelte Grenzflächenabbildungen mit unterschiedlichen Abbildungsparametern simultan mit Simulationen verglichen wurden.

\subsubsection{Die Rolle der Volumenfehlpassung}

Um der Frage des Einflusses der Volumenfehlpassung zwischen dem kristallinen und dem amorphen Material auf die mittlere Verteilung der Atome des amorphen Materials in Grenzflächennähe nachzugehen, wurde für diese Arbeit sowohl die Grenzfläche zwischen amorphem Germanium und kristallinem Silizium als auch die zwischen amorphem und kristallinem Silizium untersucht.

Die in Kapitel 4.1 erzielten Ergebnisse für die zweidimensionalen Verteilungsfunktionen werden noch einmal in Abbildung 5.1(a) und (b) zusammengefasst und im Folgenden im Hinblick auf die in der Einleitung erörterten Fragestellungen miteinander verglichen und diskutiert. 
(a)

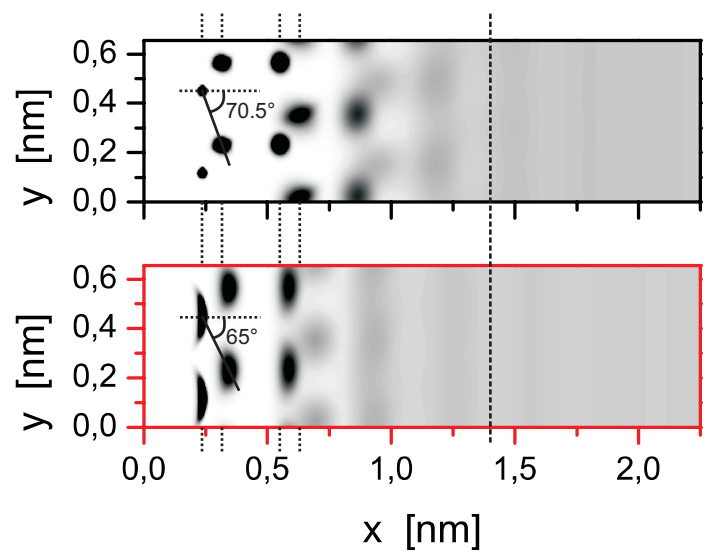

(b)



Abbildung 5.1 - Vergleich der (a) zweidimensionalen Verteilungsfunktionen und (b) der eindimensionalen Profile der atomaren Dichte des amorphen Siliziums der a-Si/c-Si- (schwarz) und des amorphen Germaniums der a-Ge/c-Si-Probe (rot). In beiden Abbildungsteilen ist der mit zunehmender Entfernung von der Grenzfläche deutlichere Einfluss der unterschiedlichen mittleren Orientierung der Bindungen und der Bindungslänge zu erkennen. So verschieben sich die atomaren Lagen der Verteilungsfunktion des amorphen Germaniums gegenüber denen des amorphen Siliziums offensichtlich nach hinten (vertikale, gepunktete Striche). Des Weiteren ist die Verteilung des amorphen Germaniums in der ersten atomaren Lage entlang der Grenze deutlich verbreitert. Die gestrichelte Linie bezeichnet in beiden Abbildungsteilen die für beide Proben bestimmte identische Ausdehnung des Übergangsbereiches von $1.4 \mathrm{~nm}$. 


\subsubsection{Der Einfluß auf die Standardabweichung von der mittleren Bindungsorientierung in der ersten amorphen Atomlage und die mittlere Bindungsorientierung}

Die für diese Arbeit berechneten Parameter der zweidimensionalen Verteilungsfunktion für das amorphe Germanium der a-Ge/c-Si-Probe präzisieren die bereits von BORGARDT ET AL. ${ }^{[13]}$ bestimmten Werte. So ergab sich für die Standardabweichung der Verteilung der Abweichungen von der mittleren Bindungsorientierung in der ersten Atomlage ein Wert von $\sigma_{\theta 1}=11.3^{\circ}$, während BORGARDT ET AL. einen Wert von $\sigma_{\theta 1}=8.8^{\circ}$ berechneten. Der Unterschied ist in der Anzahl der verwendeten Parameter für die Konstruktion der zweidimensionalen Verteilungsfunktion begründet. Die rekursive Art der Konstruktion führt bei der Benutzung von 2 Parametern, wie von BorGARDT ET AL. vorgenommen, zu einem starken Einfluss der Abweichungen jenseits der ersten Atomlage auf die angepasste Standardabweichung in der ersten Lage. Die Verwendung einer größeren Anzahl von Parametern führt somit zu einer unabhängigeren und infolgedessen besseren Beschreibung der Abweichungen von den mittleren Bindungsorientierungen der einzelnen Atomlagen. Folglich ist der in dieser Untersuchung berechnete Wert von $\sigma_{\theta 1}=11.3^{\circ}$ unter Verwendung von 6 Parametern für die Konstruktion der zweidimensionalen Verteilungsfunktion als verlässlicher zu bewerten. Beide Werte sind aber vergleichbar mit dem Wert der Standardabweichung der Bindungswinkelverteilung für reines amorphes Germanium von $9.7^{\circ}[26]$.

Für die mittlere Orientierung der Bindungen in der ersten atomaren Lage relativ zur [111]-Richtung (Winkel $\varphi$ ) ergab sich aber in dieser Untersuchung analog zu der von BorGARDT ET AL. ${ }^{[13]}$ nicht mehr $70.5^{\circ}$, wie im Fall des ungestörten Diamantgitters, sondern nur noch $65^{\circ}$.

Für das Materialsystem des amorphen Siliziums auf kristallinem Silizium hingegen ergab sich für die mittlere Orientierung der Bindungen in der ersten amorphen Atomlage wie im Fall des ungestörten Diamantgitters $70.5^{\circ}$ und für die Standardabweichung der Verteilung der Abweichungen vom mittleren Bindungswinkel der ersten Atomlage ein Wert von $\sigma_{\theta 1}=1.9^{\circ}$, der deutlich kleiner ist als bei der a-Ge/c-Si-Probe.

Diese Ergebnisse legen den Schluß nahe, dass die Unterschiede in den Werten für $\sigma_{\theta 1}$ und $\varphi$ der beiden Proben als Reaktion des amorphen Materials auf die Volumenfehlpassung zu interpretieren sind. Diese Messungen bestätigen die Aussage von BorGardT ET AL. ${ }^{[13]}$, dass das amorphe Germanium beim Aufwachsen auf das Silizium genauso wie dünne kristalline Materialien, die eine Fehlpassung zum Substrat aufweisen, eine tetragonale, elastische Verzerrung erfährt.

\subsubsection{Der Einfluß auf die Weite des Übergangsbereiches}

Um die Weite des Einflussbereiches der kristallinen Ordnung auf das amorphe Material in Grenzflächennähe abschätzen zu können, wurde analog zu BORGARDT ET AL. ${ }^{[13]}$ der 
Bildvergleich sowohl für die angepasste zweidimensionale Verteilungsfunktion als auch für eine modifizierte Version dieser Funktion, bei der ab einer gewissen Entfernung von der Grenzfläche eine homogene Verteilung anstelle der vorhandenen Modulation der atomaren Dichte angesetzt wurde, durchgeführt. Veränderte sich das Bildvergleichsmaß nur noch um 1\%, so wurde diese Position als Ende des Einflussbereiches definiert ${ }^{[13]}$.

Wie sich in der vorliegenden Untersuchung zeigte, hat die Verwendung von 6 Parametern anstelle von 2 für die Konstruktion der Verteilungsfunktion des amorphen Germaniums der a-Ge/c-Si-Probe keinen Einfluss auf die Weite des Übergangsbereiches, so dass sich analog zu BorGARDT ET AL. ${ }^{[13]}$ ein Wert von $\approx 1.4 \mathrm{~nm}$ ergab.

Erstmals konnte im Rahmen dieser Arbeit auch ein experimenteller Wert für die Weite des amorphen Bereiches mit modifizierter amorpher Struktur für das Materialsystem amorphes Silizium auf kristallinem Silizium bestimmt werden. Es ergab sich auch hier ein Wert von $\approx 1.4 \mathrm{~nm}$. Wie bereits in Abschnitt 2.1 diskutiert, schwanken die theoretischen Vorhersagen hierfür abhängig von der verwendeten Methode und dem benutzten Maß für Ordnung von $0.3 \mathrm{~nm}^{[38]}$ bis zu 1.1.nm ${ }^{[41]}$. Das experimentelle Ergebnis stützt somit das Ergebnis von DA Silva ET AL. ${ }^{[4]}$, die mithilfe von Monte-Carlo-Simulationen einen Wert von 1.1nm erhalten haben und für die Bestimmung der Ausdehnung des Übergangsbereiches die mittlere Abweichung vom theoretischen Bindungswinkel benutzten.

\subsubsection{Die Lokalisierung der Atomverteilung}

Auch wenn die Ausdehnung des Übergangsbereiches trotz des Unterschieds in der Volumenfehlpassung für beide Proben identisch ist, so besteht doch ein qualitativer Unterschied in der Lokalisierung der Atomverteilung des amorphen Materials in den ersten Atomlagen. In Abbildung 5.1 ist gut zu erkennen, dass diese in der Verteilungsfunktion des amorphen Siliziums der a-Si/c-Si-Probe in den ersten Atomlagen deutlich stärker ausgeprägt ist als in der Verteilungsfunktion des amorphen Germaniums der a-Ge/c-SiProbe. Dieses Verhalten wird durch die graphische Darstellung in Abbildung 5.2 bestätigt, in dessen Abbildungsteil (b) für die ersten 3 bzw. 4 Atomlagen der Verteilungsfunktionen beider Proben die Fläche, innerhalb derer sich ein Atom in der Verteilung mit $66 \%$ Wahrscheinlichkeit befindet, gegen die Atomlage aufgetragen ist. Diese Fläche wird im Folgenden als 66\%-Fläche bezeichnet. Weitere Atomlagen wurden aufgrund der Überlappung der Verteilungen für die Atomlagen nicht ausgewertet.

Es ist deutlich zu erkennen, dass innerhalb der ersten drei Atomlagen die Atome des amorphen Siliziums der a-Si/c-Si-Probe im Gegensatz zu denen der a-Ge/c-Si-Probe im Mittel nur geringfügig von den Positionen der durch das Substrat induzierten kristallinen Ordnung abweichen. Das amorphe Silizium kann also diese Ordnung besser übernehmen als das amorphe Germanium, was bereits in der ersten Atomlage eine deutliche Aufweitung der atomaren Verteilung zeigt.

Beim Übergang zur vierten Atomlage jedoch nimmt die 66\%-Fläche für die Verteilungsfunktion des amorphen Siliziums der a-Si/c-Si-Probe im Verhältnis zu den Flächen 
(a)

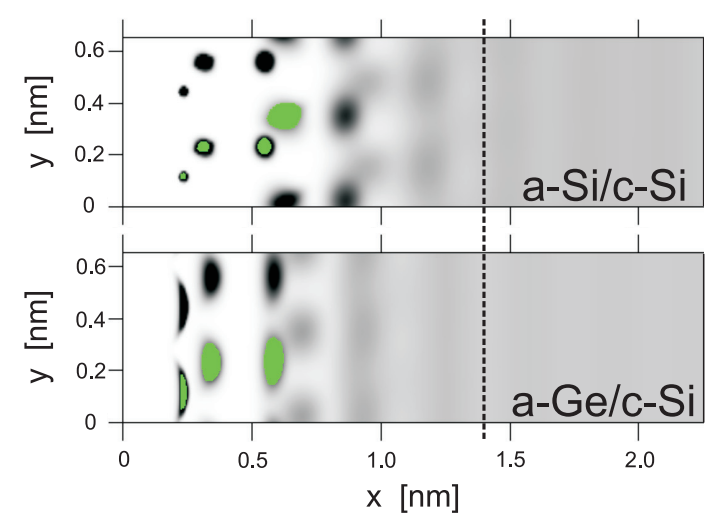

(b)

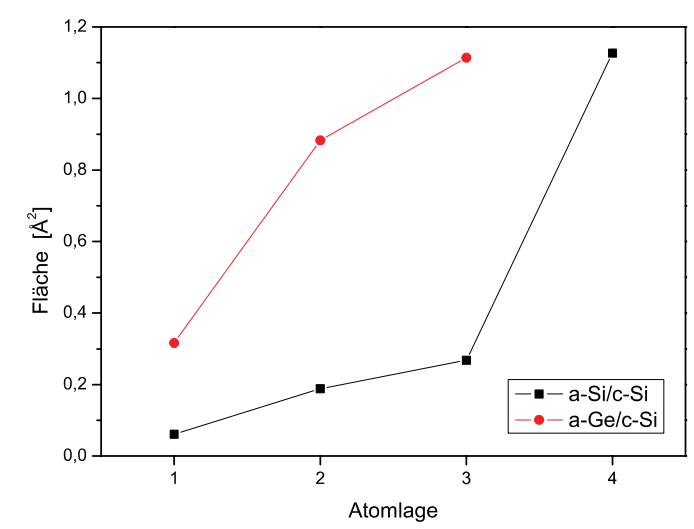

Abbildung 5.2 - Berechnete Fläche, innerhalb derer sich ein Atom mit $\approx 66 \%$ Wahrscheinlichkeit befindet. (a) Graphische Darstellung der 66\%-Fläche für die ersten vier Atomlagen der Verteilungsfunktion des amorphen Siliziums (oben) und der ersten drei Atomlagen der Verteilungsfunktion des amorphen Germaniums (unten). Weitere Atomlagen wurden aufgrund der Überlappung der Verteilungen nicht ausgewertet. Der vertikale Strich zeigt an, wo der Übergangsbereich endet. (b) Auftragung der 66\%-Fläche gegen die Atomlage. Es zeigt sich, dass die Verteilung der atomaren Dichte des amorphen Siliziums in den ersten drei Atomlagen wesentlich stärker ausgeprägt ist.

\begin{tabular}{cccccccc}
\hline \hline Probe & $\varphi$ & $\sigma_{\theta 1}$ & $\sigma_{\theta 2}$ & $\sigma_{\theta 3}$ & $\sigma_{\theta 4}$ & $\alpha_{1}$ & $\alpha_{2}$ \\
\hline $\mathrm{a}-\mathrm{Si} / \mathrm{c}-\mathrm{Si}$ & $70.5^{\circ}$ & $1.9^{\circ}$ & $3.9^{\circ}$ & $3.8^{\circ}$ & $10.7^{\circ}$ & $3.3^{\circ}$ & $6.2^{\circ}$ \\
$\mathrm{a}-\mathrm{Ge} / \mathrm{c}-\mathrm{Si}$ & $65^{\circ}$ & $11.3^{\circ}$ & $6.0^{\circ}$ & $10.3^{\circ}$ & $16.2^{\circ}$ & $10.9^{\circ}$ & $3.0^{\circ}$ \\
\hline \hline
\end{tabular}

Tabelle 5.1 - Zusammenfassung der besten Parametersätze für die zweidimensionale Verteilungsfunktion des amorphen Siliziums der a-Si/c-Si-Probe und des amorphen Germaniums der a-Ge/c-Si-Probe nach der iterativen Bildanpassung beider Defokusserien.

der vorigen Atomlagen deutlich zu. Dieses Verhalten ist auch gut an den Parametern der Verteilungsfunktion zu erkennen (s. auch Tab. 5.1). Während die ersten drei Werte der Standardabweichungen der a-Si $/$ c-Si-Probe mit $\sigma_{\theta 1}=1.9^{\circ}, \sigma_{\theta 2}=3.9^{\circ}$ und $\sigma_{\theta 3}=3.8^{\circ}$ klein und die Atome folglich noch stark lokalisiert sind, steigt der Wert für die vierte Atomlage mit $\sigma_{\theta 4}=10.7^{\circ}$ deutlich an. Die Lokalisierung nimmt also ab dieser Lage merklich ab. Dasselbe Verhalten ist, wenn auch in etwas abgeschwächter Form, auch für die Verteilung des amorphen Germaniums der a-Ge/c-Si-Probe zu erkennen, allerdings nur anhand der Parameter der Verteilungsfunktion, da aufgrund der Überlappung der Atomverteilung der Atomlagen die 66\%-Fläche oberhalb der dritten Lage nicht bestimmt werden konnte. In Tabelle 5.1 ist aber ebenso ein deutlicher Anstieg der Standardabweichung der dritten Atomlage zu erkennen. Dies bedeutet, dass die ersten drei Atomlagen noch einen großen Einfluß der kristallinen Ordnung des Substrats spüren, während er oberhalb von $\approx 0.6 \mathrm{~nm}$ zwar immer noch vorhanden ist, aber offensichtlich nachlässt. 


\subsubsection{Untersuchung der Homogenität der zweidimensionalen Verteilungsfunktion entlang der Grenzfläche}

Um eine systematische Untersuchung der Homogenität der Verteilungsfunktion entlang der Grenzfläche zu ermöglichen, musste für diese Arbeit die Untersuchungsmethode von BORGARDT ET AL. ${ }^{[10,11,13]}$ modifiziert werden. So wurde zunächst die Frage der lateralen Auflösung geklärt, d.h. wie groß die mindestens notwendige Ausdehnung des Mittelungsbereiches ist. Obwohl für die in dieser Arbeit verwendete statistische Beschreibung des strukturellen Übergangs die Ausdehnung des Mittelungsbereiches eigentlich so groß wie möglich gewählt werden sollte, konnte aus der Theorie der APP-Näherung ${ }^{[11]}$ eine robuste Annahme für die minimale Größe des Mittelungsbereiches abgeleitet werden.

Um weiterhin entscheiden zu können, ob Unterschiede zwischen zwei Verteilungsfunktionen, welche sich aus unterschiedlichen Mittelungsbereichen ergeben, signifikant sind, wurde die Bootstrap-Methode ${ }^{[27-29]}$ in das bestehende Untersuchungsverfahren integriert. Dieses Verfahren ermöglicht erstmals die Bestimmung der Fehler in jedem Bildpunkt der zweidimensionalen Verteilungsfunktion, welche ihre Ursache in den Varianzen der Intensitäten der gemittelten experimentellen Grenzflächenabbildungen haben.

\subsubsection{1 a-Ge/c-Si-Probe}

Für eine graphische Darstellung der Ergebnisse sei noch einmal auf Abbildung 4.18 in Kapitel 4.2.1 auf Seite 76 verwiesen.

Anhand der erzielten Ergebnisse konnte gezeigt werden, dass signifikante strukturelle Variationen entlang der Grenzfläche zwischen amorphem Germanium und kristallinem Silizium erst ab der zweiten Atomlage auftreten. Die atomare Verteilung innerhalb der ersten Atomlage blieb im Rahmen der Fehler konstant, was auf homogene Eigenschaften dieser Lage auf der Skala des Bildausschnitts hinweist. Die Ausdehnung des Mittelungsbereiches betrug bei dieser Untersuchung $\approx 10 \mathrm{~nm}$. Das Verhalten der ersten Atomlage kann analog zu der Interpretation von OHDOMARI ET AL. ${ }^{[03]}$ durch den ordnenden Effekt der aus der Si(111)-Oberfläche senkrecht herausragenden Si[111]-Bindungen erklärt werden.

Unterschiede in den strukturelle Variationen jenseits der dritten Atomlage sind - wenn überhaupt vorhanden - so klein, dass sie durch den Vergleich zweier Verteilungsfunktionen nicht signifikant nachgewiesen werden konnten. Hier sind die Verteilungsfunktionen bereits zu wenig um ihre lokalen Maxima lokalisiert.

Des Weiteren zeigt sich sowohl anhand der Abfolge der 41 Profile der atomaren Dichte als auch der Untersuchung der 66\%-Flächen aller 41 Verteilungsfunktionen, dass in dem mittleren Teil des abgebildeten Bereiches die Germaniumatome im Übergangsbereich im Mittel stärker lokalisiert sind als am oberen bzw. unteren Teil. Ein Grund für dieses Verhalten können z.B. strukturelle Inhomogenitäten entlang der Grenzfläche sein, die lokal in dem mittleren Teil des abgebildeten Bereiches der Grenzfläche für eine weiterreichende 
Ordnung in der mittleren Atomverteilung im Übergangsbereich sorgen.

\subsubsection{2 a-Si/c-Si-Probe}

Im Gegensatz zu der a-Ge/c-Si-Probe weist die Grenzfläche zwischen kristallinem und amorphem Silizium lateral deutliche strukturelle Inhomogenitäten auf (kleine Inseln bzw. Stufen, s. auch Abb. 4.1 auf Seite 53). Eine Beschreibung der grenzflächeninduzierten Ordnung durch eine statistische zweidimensionale Verteilungsfunktion ist aber nur dann sinnvoll, wenn im Mittelungsbereich an der Grenzfläche keine strukturellen Inhomogenitäten zu erkennen sind. Diese führen sonst zu großen Varianzen in den Intensitäten der gemittelten Grenzflächenabbildungen und somit auch zu einer großen Unsicherheit in der zweidimensionalen Verteilungsfunktion. Infolgedessen müsste die Ausdehnung des Mittelungsbereiches reduziert werden, in dem vorliegenden Fall sogar kleiner als die minimal für die statistische Beschreibung notwendige Ausdehnung. Aufgrund dessen konnte diese Grenzfläche nicht mithilfe des in dieser Arbeit verfolgten Ansatzes systematisch untersucht werden. Der in Abschnitt 4.1 untersuchte Bereich der Grenzfläche ist der einzige Bereich, der über dem notwendigen Mittelungsbereich keine deutlichen strukturellen Inhomogenitäten zeigt.

Da durch die Probenpräparation sichergestellt wurde, dass die Substratoberfläche vor der Materialdeposition atomar glatt war, liegt die Ursache der starken strukturellen Inhomogenität der Grenzfläche höchstwahrscheinlich in dem verwendeten Depositionsverfahren selbst. Im Gegensatz zu der a-Ge/c-Si-Probe ist das amorphe Silizium nicht mittels Molekularstrahlepitaxie sondern, wie bereits oben erwähnt, durch Sputtern auf dem Substrat abgeschieden worden. Dies bedeutet zum einen, dass die Bedampfung nicht unter UHV-Bedingungen stattgefunden hat, und zum anderen, dass die mittlere kinetische Energie der deponierten Atome in der Größenordnung von $10 \mathrm{eV}$ liegt, während sie bei der MBE weniger als $1 \mathrm{eV}$ beträgt ${ }^{[119]}$. Gründe für strukturelle Inhomogenitäten könnten also z.B. durch das geringe Vakuum in der Depositionskammer an der Grenzfläche eingebaute Atome des Restgases, Oberflächenerosion durch hohe Energie der deponierten Atome oder Tieftemperaturepitaxie sein. Diese Frage kann aber letztlich anhand der vorliegenden Daten nicht beantwortet werden und muss somit offen bleiben.

\subsection{Ausblick}

Die in dieser Arbeit verwendete Methode des iterativen Bildserienvergleichs mittlerer experimenteller und simulierter elektronenmikroskopischer Abbildungen unter Verwendung der zweidimensionalen Verteilungsfunktion $\rho(x, y)$ für die Struktur des amorphen Materials in Grenzflächennähe eröffnet eine Vielzahl möglicher weiterer Anwendungen.

So stellt die Beschreibung der atomaren Grenzflächenstruktur zwischen Silizium und Siliziumdioxid die technologisch sicherlich bedeutendste Herausforderung dar. PLIKAT ${ }^{[24]}$ 
hat bereits gezeigt, dass es eine stabilisierte, ca. 2nm dicke $\mathrm{SiO}_{2}$-Schicht an der Grenzfläche gibt, so dass die starke Strahlenschädigung des Oxids durch den Elektronenbeschuß im Elektronenmikroskop kein Problem mehr darstellt. Eine Schwierigkeit liegt aber noch in der ungeklärten Stöchiometrie des Siliziumdioxids an der Grenzfläche, was die Konstruktion von $\rho(x, y)$ erschwert.

Um den Einfluß der Fehlpassung systematisch zu erforschen, könnte die Untersuchung des Systems $\mathrm{Si}_{1-x} \mathrm{Ge}_{x}(\mathrm{x}=0, \ldots, 1)$ weitere Aufschlüsse liefern. Durch die Analyse einer Metall/Silizium-Legierung kann des Weiteren geprüft werden, wie sich der vorwiegende Bindungscharakter auf die zweidimensionale Verteilungsfunktion auswirkt.

Die in dieser Arbeit etablierte Methode zur Untersuchung der Homogenität der Verteilungsfunktion entlang der Grenzfläche bietet ferner die Möglichkeit, die Variationen in der atomaren Struktur des Übergangs entlang der Grenzfläche zu quantifizieren und einen Zusammenhang mit physikalischen Eigenschaften herzustellen. Auf diese Weise können laterale strukturelle Variationen z.B. in dünnen Tunnelbarrieren eines TMRElements oder in der Oxidschicht einer MOS-Struktur mit physikalischen Eigenschaften des Bauelements verglichen werden.

Es gibt eine Reihe weiterer Möglichkeiten apparativer Art, die Methode zu verbessern. Die Verwendung einer größeren CCD-Kamera $(2048 \times 2048$ Bildpunkte) würde es ermöglichen, einen größeren Probenbereich abzubilden und somit Korrelationen in den Verteilungsfunktionen entlang eines weiteren Bereiches der Grenzfläche zu untersuchen. Die Benutzung einer Kamera, die schneller ausgelesen werden kann, würde wiederum erlauben, die Probendrift während der Aufnahme der Defokusserie weiter zu reduzieren. Um den Einfluss der Delokalisierung zu minimieren, könnten $C_{S}$-korrigierte Mikroskope Verwendung finden ${ }^{[120,121]}$ oder durch die Objektwellenrekonstruktion berechnete Objektwellen untersucht werden ${ }^{[73-76]}$. 
Anhänge 

$\Gamma_{\text {Anhang }} \mathrm{A}_{2}$

\section{Vorläufige Bestimmung der Defokusschrittweite $\delta \Delta f$}

Ein gängiges Verfahren zur Bestimmung der Mikroskopparameter, also z.B. der Defokussierung und der sphärischen Aberration, ist die Auswertung von Diffraktogrammen dünner amorpher Proben ${ }^{[122-124]}$. Ausgangspunkt für diese Analysen ist, dass die Probe als schwaches Phasenobjekt betrachtet werden kann, d.h. dass ein einfallender Elektronenstrahl beim Durchlaufen der Probe relativ zu einem Elektronenstrahl, der dieselbe Strecke im Vakuum durchläuft, lediglich einen Phasenschub « 1 aufgeprägt bekommt. Voraussetzung für die Gültigkeit dieser Näherung ist zum einen, dass der ungebeugte Elektronenstrahl wesentlich stärker als die abgebeugten Strahlen ist, so dass Interferenzen zwischen den abgebeugten Strahlen vernachlässigt werden können. Zum anderen muss die Schärfentiefe größer als die Probendicke sein (Projektionsnäherung).

In diesem Fall zeigt sich im Diffraktrogramm eine Abfolge von Ringen, deren Anzahl und Abstände zum Ursprung ein Maß für den Defokus $\Delta f$ und die sphärische Aberration $C_{S}$ sind. Dabei sind die Minima im azimuthal gemittelten Diffraktogramm durch die Nullstellen des Imaginärteils der Transferfunktion, also durch den Sinus der Aberrationsfunktion $\chi(\vec{q})$ gegeben (s. auch Abb. A.1).

$$
\begin{aligned}
\text { Transferfunktion: } & \mathrm{T}(\vec{q})=\exp \{i \chi(\vec{q})\}=\cos \chi(\vec{q})+i \sin \chi(\vec{q}) \\
\text { Aberrationsfunktion: } & \chi(\vec{q})=\pi\left(\frac{C_{S} q^{4} \lambda^{3}}{2}+\Delta f q^{2} \lambda\right)
\end{aligned}
$$

Dabei steht $C_{S}$ für die sphärische Aberration, $\lambda$ für die Wellenlänge der Elektronen, $\Delta f$ für den Defokus und $\vec{q}$ für die Raumfrequenz. Alle anderen Aberrationen, wie z.B. der 2und 3-zählige Astigmatismus oder die axiale Koma, werden im Folgenden vernachlässigt. Bei dem für die vorliegende Untersuchung verwendeten Mikroskop CM200-FEG-UT der Firma Philips beträgt der Wert für $C_{S}=0.48 \mathrm{~mm}$ und die Beschleunigungsspannung 
Abbildung A.1 - Obere Hälfte: Diffraktogramm einer amorphen Siliziumprobe. Deutlich ist die Abfolge von Ringen zu erkennen. Untere Hälfte: Mittels der im Text beschriebenen Methode angepasster Betrag des Sinus der Aberrationsfunktion.

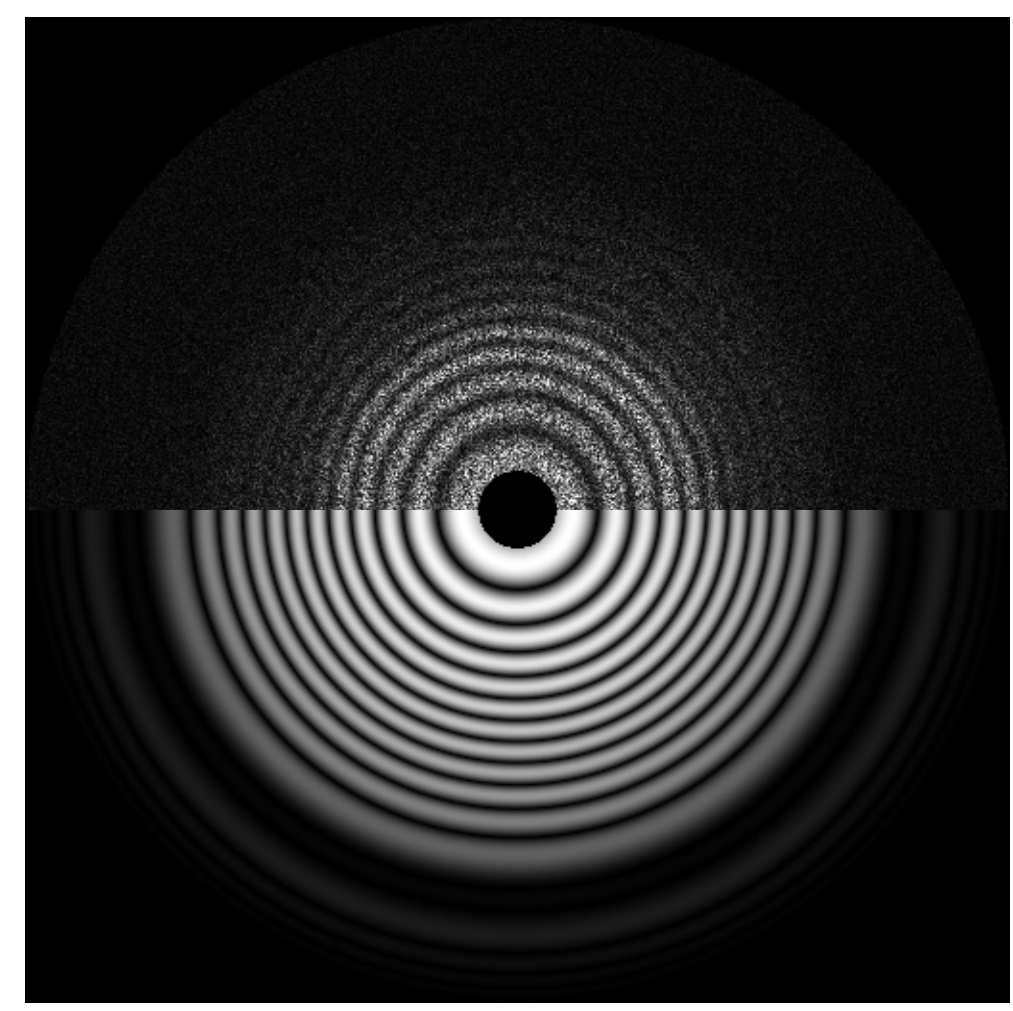

von $200 \mathrm{kV}$ sorgt für eine Wellenlänge der Elektronen von $\lambda=2.51 \mathrm{pm}$.

GIBSON ${ }^{[125]}$ hat sich mit den Voraussetzungen für die Gültigkeit der Näherung des schwachen Phasenobjekts und deren Zusammenbruch bei dünnen, amorphen Proben auseinandergesetzt. Anhand von Simulationen von Abbildungen amorpher Germaniumfilme konnte er zeigen, dass bei zunehmender Probendicke zuerst die Annahme der notwendigen Tiefenschärfe verletzt wird. Solange die Probendicke aber einen kritischen Wert von

$$
t_{\mathrm{krit}}=\frac{1}{4 \lambda q_{\max }^{2}}
$$

nicht überschreitet, ist die Tiefenschärfe groß genug. $q_{\max }$ ist dabei die größte ins Bild übertragene Raumfrequenz*.

Für das Informationslimit des verwendeten Mikroskops $\left(q_{\text {info }} \approx 8 \mathrm{~nm}^{-1}\right)$ ergibt sich somit als größte mögliche Probendicke $t_{\text {krit }}=1.6 \mathrm{~nm}$. Deswegen stellt der sehr dünne amorphe Probenrand ein geeignetes Objekt für die Analyse dar.

Die Bestimmung der Positionen der experimentellen Minima geschieht mittels einer parabolischen Interpolation der drei das Minimum umgebenden Punkte ${ }^{[29]}$. Diese so berechneten Positionen werden mit den theoretischen Nulldurchgängen des Sinus der Aberrationsfunktion verglichen, deren Positionen sich entsprechend Gleichung A.1 und

${ }^{*}$ Bei Verwendung einer Aperturblende ist natürlich dieser Wert für $q_{\max } \mathrm{zu}$ verwenden. 
Gleichung A.2 zu

$$
\begin{aligned}
e^{i \chi(q)} & =\cos \chi(q)+i \sin \chi(q) \\
\sin \chi(q) & =\sin \left\{\pi\left(\frac{C_{s} q^{4} \lambda^{3}}{2}+\Delta f q^{2} \lambda\right)\right\}=0 \\
\Rightarrow \chi(q) & =n \cdot \pi ; \quad n \in \mathbb{N} \\
\Rightarrow q(n) & =\sqrt{-\frac{\Delta f}{C_{s} \lambda^{2}} \pm \sqrt{\left(\frac{\Delta f}{C_{s} \lambda^{2}}\right)^{2}+\frac{2}{C_{s} \lambda^{3}} \cdot n}}
\end{aligned}
$$

berechnen.

In der numerischen Implementation werden eine Vielzahl möglicher Nullstellen für einen vorgegebenen Parameterbereich des Defokus berechnet und mit den experimentellen Minimapositionen verglichen. Schließlich wird derjenige Defokus ausgewählt, der das kleinste $\chi^{2}$ aufweist (s. auch Abb. A.1):

$$
\chi_{\Delta f}^{2}=\sum_{i=1}^{K}\left(q_{i}^{\exp }-q_{i}^{\text {theo }}\right)^{2} \quad \text { für } K \text { identifizierte experimentelle Minima }
$$

Mittels der beschriebenen Methode wurden die Defoki für neun experimentelle Defokusserien im Defokusbereich von $\Delta f=-125$ bis $-370 \mathrm{~nm}$ bestimmt (s. Abb. A.2). Die instrumentelle Defokusschrittweite war dabei dieselbe wie für die Defokusserie der a-Si/c-Si- und a-Ge/c-Si-Probe.

Nach der Bestimmung der Defokuswerte wurden mittels linearer Regression der Startdefokus $\Delta f_{1}$ und die Defokusschrittweite $\delta \Delta f$ bestimmt. Abbildung A.2 zeigt die lineare Anpassung, und Tabelle A.1 fasst die Ergebnisse zusammen. Als Mittelwert für die Defokusschrittweite ergab sich 7.0(4)nm.

Im Rahmen dieser Auswertung wurde keine Fehlerabschätzung für die einzelnen Defokuswerte durchgeführt, da die durch diese Untersuchung bestimmte Defokusschrittweite lediglich als Startwert in den iterativen Bildvergleich von Simulation und Experiment einging (siehe Abschnitt 3.2.3.2). 


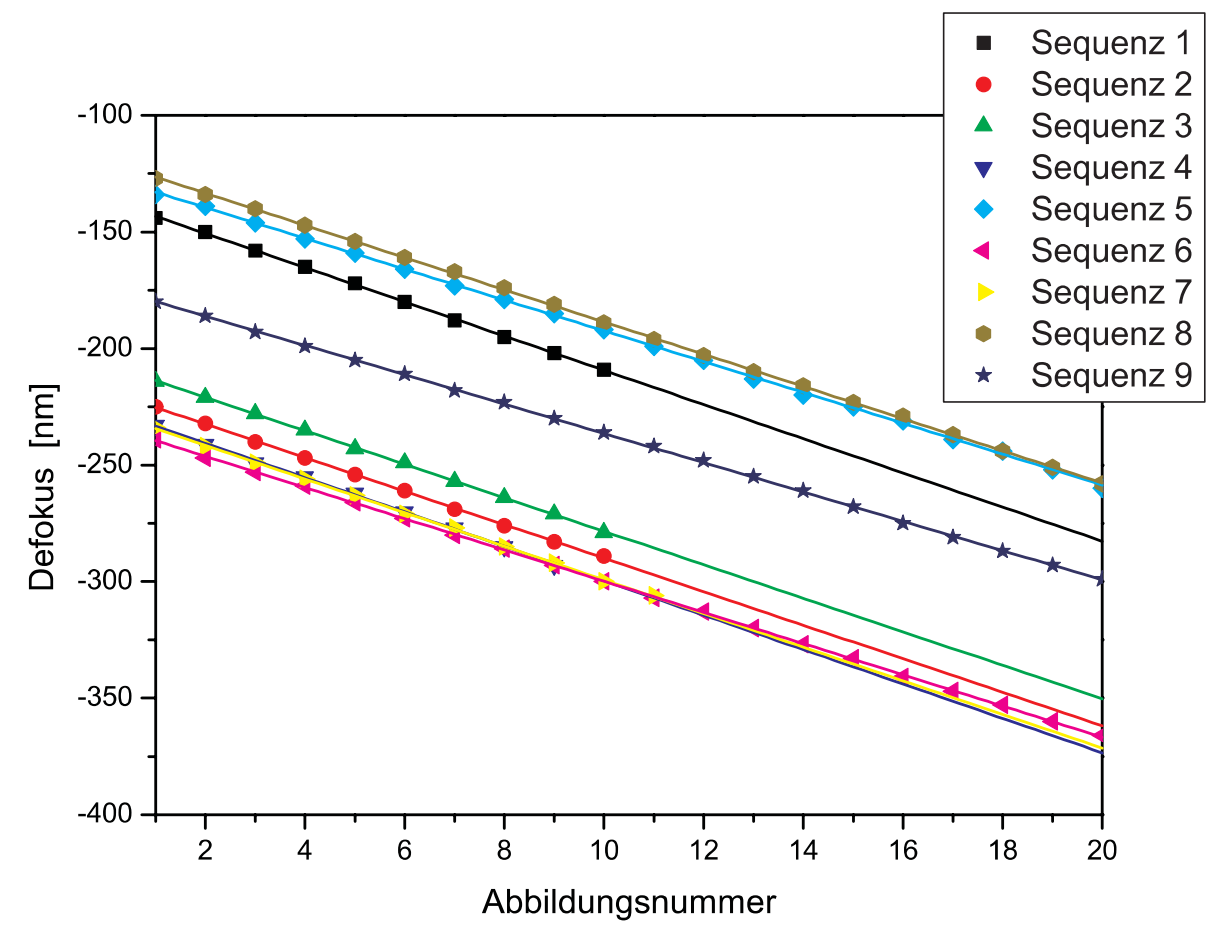

Abbildung A.2 - Graphische Auftragung der Anpassung der Defokuswerte für neun experimentelle Defokusserien.

\begin{tabular}{ccc}
\hline \hline Defokusserie & Startdefokus $\Delta f_{1}$ & Defokusschrittweite $\delta \Delta f[\mathrm{~nm}]$ \\
\hline Sequenz 1 & -135.9 & 7.3 \\
Sequenz 2 & -218.1 & 7.2 \\
Sequenz 3 & -206.5 & 7.2 \\
Sequenz 4 & -225.9 & 7.4 \\
Sequenz 5 & -126.3 & 6.6 \\
Sequenz 6 & -232.8 & 6.7 \\
Sequenz 7 & -227.2 & 7.2 \\
Sequenz 8 & -119.5 & 6.9 \\
Sequenz 9 & -173.5 & 6.3 \\
\hline & Mittelwert & $7.0(4)$ \\
\hline \hline
\end{tabular}

Tabelle A.1 - Mittels der im Text beschriebenen Methode bestimmte Defokusschrittweite für neun experimentelle Defokusserien. 


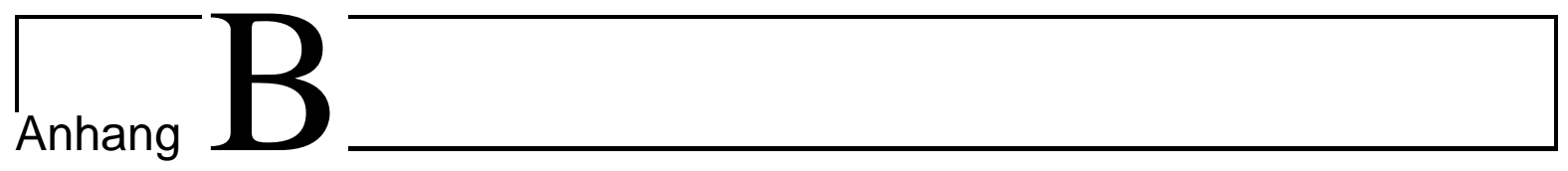

\section{Präparation der Proben}

Ziel der Probenpräparation war die Herstellung einer atomar glatten, unrekonstruierten Oberfläche des Substrats vor der Deposition. Wie bereits des öfteren in dieser Arbeit erwähnt, entstammen die verwendeten Abbildungen der a-Ge/c-Si-Probe der Dissertation von PliKaT ${ }^{[24]}$. In seiner Arbeit beschäftigte er sich ausführlich mit den Anforderungen an die Probenherstellung und der endgültigen Durchführung, die auf einem Verfahren von Suhren ET AL. ${ }^{[118]}$ basiert. Da für die neu erstellte a-Si/c-Si-Probe dieselbe Vorbehandlung gewählt wurde, soll dieses Verfahren hier nicht wiederholt werden. Der interessierte Leser sei auf die Arbeiten von PLIKAT ${ }^{[24]}$ und Suhren ET AL. ${ }^{[18]}$ verwiesen.

Die a-Si/c-Si-Probe wurde in Zusammenarbeit mit Dr. Lars Korte aus dem HahnMeitner-Institut in Berlin angefertigt, während die a-Ge/c-Si-Probe einer Zusammenarbeit mit Dr. Thomas Wagner vom Max-Planck-Institut für Metallforschung in Stuttgart entstammt.

\section{Deposition}

\section{Amorphes Germanium}

Die Bedampfung des Substrats mit Germanium erfolgte mittels Molekularstrahlepitaxie bei Raumtemperatur. Die mittlere Aufdampfrate lag bei $3.3 \mathrm{~nm} / \mathrm{min}$ und die gesamte Schichtdicke betrug 100nm. Die Aufdampfdauer lag somit bei 30min. In der Hauptkammer lag nach dem Einschleusen und kurz vor dem Bedampfen der Probe ein Druck von $p=7 \times 10^{-10}$ mbar vor. Dieser stieg während des Bedampfungsvorgangs auf $p=7 \times 10^{-9}$ mbar nach einer Schichtdicke von $d_{\mathrm{Ge}}=50 \mathrm{~nm}$ und $p=1.2 \times 10^{-8} \mathrm{mbar}$ nach $d_{\mathrm{Ge}}=100 \mathrm{~nm}$. Diese Daten sind der Dissertation von PLIKAT ${ }^{[24]}$ entnommen. 
(a)

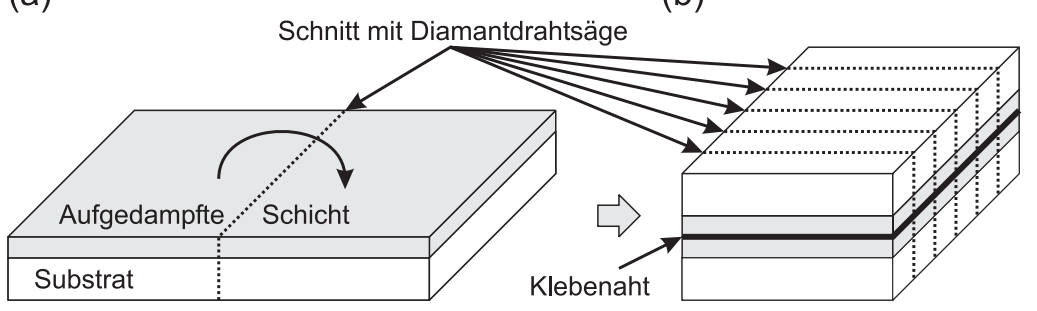

(c)

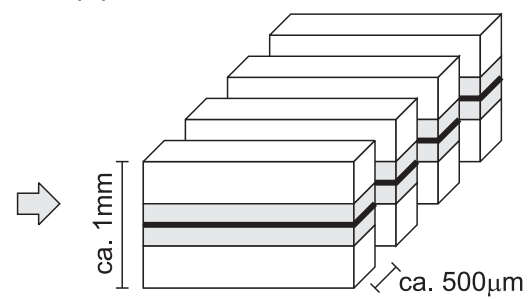

Abbildung B.1 - Schema zur Präparation von TEM-Folien I. (a) Die bedampfte Probe wird mittels einer Diamantdrahtsäge in zwei Teile gesägt. (b) Die beiden Teile werden aufeinanderge-

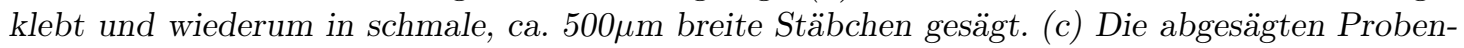
stäbchen.

\section{Amorphes Silizium}

Das amorphe Silizium wurde auf das kristalline Substrat ebenfalls bei Raumtemperatur aufgesputtert. Der gesamte Depositionsprozess dauerte $7 \mathrm{~min}$ bei einer Rate von 14.3nm, so dass 100nm Material auf dem Substrat abgeschieden wurden. Der Basisdruck innerhalb der Kammer betrug $p=1 \times 10^{-7}$ mbar. Als Prozessgas wurde Argon bei einem Druck von $p=6 \times 10^{-2}$ mbar verwendet ${ }^{[126]}$.

\section{TEM-Präparation}

Die Präparation der Querschnittsproben basierte auf dem bei SEIBT ${ }^{[127]}$, GNAUERT ${ }^{[128]}$ oder GLANDER ${ }^{[129]}$ beschriebenen Dünnverfahren, dessen einzelne Schritte hier noch einmal kurz beschrieben werden sollen.

1. Die bedampfte Probe wurde zuerst durch Anritzen mit einem Diamantschneider in zwei Stücke gebrochen (Abbildung B.1(a)).

2. Anschließend wurden beide Probenstücke nacheinander für mehrere Minuten in einem Ultraschallbad mit deionisiertem Wasser, Aceton und wieder mit deionisiertem Wasser gereinigt.

3. Nach der Reinigung wurden die beiden bedampften Seiten mit dem Kleber M-Bond 610 der Firma Vishay Micro-Measurements zusammengeklebt, in einen Messingblock eingespannt und der Kleber für mehrere Stunden bei $130^{\circ} \mathrm{C}$ ausgehärtet.

4. Mittels einer Diamantdrahtsäge der Firma Well Diamantdrahtsägen GmbH wurde die Probe nach dem Aushärten in $500 \mu \mathrm{m}$ breite Probenstücke gesägt (Abbildung B.1(b) und (c)).

5. Ein Probenstäbchen wurde anschließend entsprechend Abbildung B.2 auf einen planparallelen Glasträger geklebt. Als Klebstoff diente hier Lakeside Brand der 


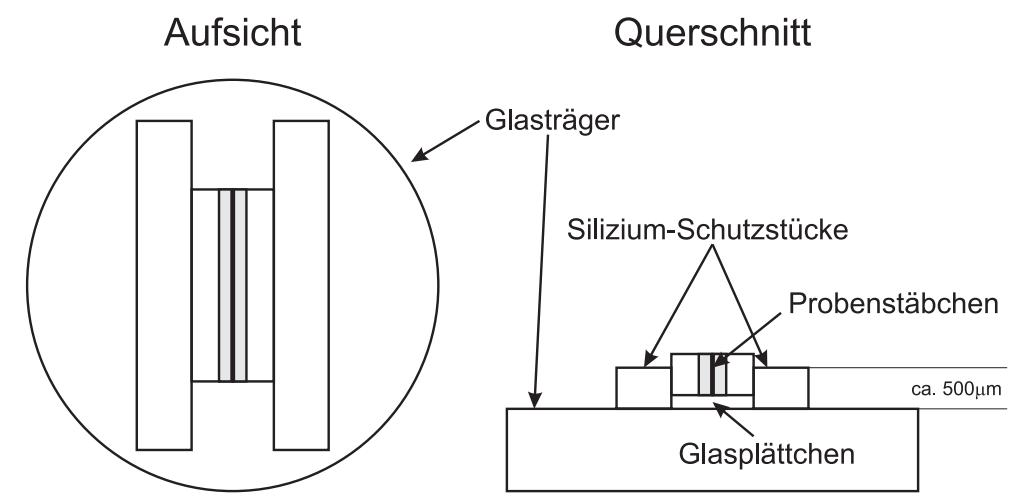

Abbildung B.2 - Schema zur Präparation von TEM-Folien II. Die Probe wird auf einen Glasträger geklebt. Links und rechts wird zur Probenstabilisierung jeweils ein ca. $500 \mu \mathrm{m}$ dickes Silizium-Schutzstück platziert. Unterhalb der Probe befindet sich ein ca. $150 \mu \mathrm{m}$ dickes Glasplättchen. Es sind die Aufsicht (links) und der Querschnitt (rechts) dargestellt.

Firma Withear Lapidary Co., der bei $130^{\circ} \mathrm{C}$ schmilzt. Links und rechts neben dem Probenstäbchen platzierte Silizium-Schutzstücke erleichtern das planparallele Abtragen der Probe und schützen die Probenkanten. Unter der Probe befindet sich zusätzlich ein Glasträger der Dicke $d=150 \mu \mathrm{m}$.

6. Mittels SiC-Schleifpapier (1000er Körnung) wurde die Probe nun auf $\approx 300 \mu \mathrm{m}$ abgeschliffen und anschließend mit $7 \mu \mathrm{m}, 1 \mu \mathrm{m}$ und $0.25 \mu \mathrm{m}$ Diamantpaste poliert. Durch Erhitzen des Glasträgers wurde der Kleber verflüssigt und die Probe mit der polierten Seite nach unten erneut aufgeklebt. Analog zur ersten Seite wurde die Probe auf $\approx 20-30 \mu \mathrm{m}$ weiter abgeschliffen und anschließend poliert. Während des Schleifprozesses war es notwendig, die Probendicke ständig mit einer Mikrometerschraube zu kontrollieren.

7. Mittels Dimethylformamid konnte die Probe vom Glasträger gelöst werden. Nach gründlicher Reinigung der Probe von Kleberückständen wurde die Probe wiederum mit M-Bond Kleber auf einen Kupferring aufgeklebt. Anschließend erfolgte der Einbau in eine Argon-Ionenätze der Firma GATAN.

8. Damit die Probe für Elektronen im Mikroskop transparent ist, darf sie nur noch einige $10 \mathrm{~nm}$ dick sein. Deswegen wurde sie in der Ionenätze mit 4.5kV Argon-Ionen unter einem Winkel von $17^{\circ}$ weiter gedünnt. Die durch diesen Beschuss amorphisierte Oberflächenschicht kann durch weiteres Dünnen unter einem geringeren Winkel von etwa $10^{\circ}$ ausreichend reduziert werden. 


\section{Literaturverzeichnis}

[1] M. Bohr, Intel's 65nm Process Technology, ftp://download.intel.com IRDS002_65nm_logic_process_100_percent.pdf, 2004, Event: Intel Developer Forum.

[2] J. S. Moodera, L. R. Kinder, T. M. Wong und R. Meservey, Large Magnetoresistance at Room Temperature in Ferromagnetic Thin Film Tunnel Junctions, Phys. Rev. Lett. 74, 3273 (1995).

[3] M. Seibt und M. Münzenberg, Struktur und Transport in Tunnelmagnetowiderstandselementen mit ultradünnen Barrieren, Projekt A14, SFB 602, Universität Göttingen.

[4] M. Münzenberg und J. S. Moodera, Superconductor-ferromagnet tunneling measurements indicate sp-spin and d-spin currents, Phys. Rev. B 70, 060402(R) (2004).

[5] D. J. Eaglesham, Semiconductor molecular-beam epitaxy at low temperatures, J. Appl. Phys. 77, 3597 (1995).

[6] F. Priolo und E. Rimini, Ion-beam-induced epitaxial crystallization and amorphization in silicon, Mater. Sci. Rep. 5, 319 (1990).

[7] P. H. Gaskell, Glasses and Amorphous Materials, Band 9 der Materials Science and Technology - A comprehensive Treatment, Kapitel 4, Seiten 175-278, VCH Weinheim, 1991.

[8] G. Möbus, R. Schweinfest, T. Gemming, T. Wagner und M. Rühle, Iterative structure retrieval technique in HREM: A comparative study and a modular program package, J. Microsc. 190, Pts 1/2, 109 (1998). 
[9] G. Möbus, Structure Determination by Quantitative High-Resolution Transmission Electron Microscopy (Q-HRTEM), in High-Resolution Imaging and Spectrometry of Material, herausgegeben von F. Ernst und M. Rühle, Band 50 der Springer Series in Materials Sciences, Kapitel 3, Seiten 69-118, Springer-Verlag, Berlin, 2003.

[10] N. I. Borgardt, B. Plikat, M. Seibt und W. Schröter, Regularities of averaged HREM-images of crystalline-amorphous interfaces, Bulletin of the Russian Academy of Sciences, Physics 61, 1544 (1997).

[11] N. I. Borgardt, B. Plikat, M. Seibt und W. Schröter, Analysis of high resolution transmission electron microscope images of crystalline-amorphous interfaces, Ultramicroscopy 90, 241 (2002).

[12] N. I. Borgardt, B. Plikat, M. Seibt und W. Schröter, The Effect of the Translational Symmetry of Crystalline Silicon on the Structure of Amorphous Germanium in the Interfacial Region, Crystallography Reports 49, 225 (2004).

[13] N. I. Borgardt, B. Plikat, W. Schröter, M. Seibt und T. Wagner, Atomic structure of the interface between silicon (111) and amorphous germanium, Phys. Rev. B 70, 195307 (2004).

[14] J. M. Cowley und A. F. Moodie, The Scattering of Electrons by Atoms and Crystals. I. A New Theoretical Approach, Acta Cryst. 10, 609 (1957).

[15] D. F. Lynch und M. A. O'Keefe, n-Beam lattice images. II. Methods of calculation, Acta Cryst. A28, 536 (1972).

[16] P. Goodman und A. F. Moodie, Numerical Evaluations of N-Beam Wave Functions in Electron Scattering by the Multi-slice Method, Acta Cryst. A30, 280 (1974).

[17] K. Ishizuka und N. Uyeda, A new theoretical and practical approach to the multislice method, Acta Cryst. A33, 740 (1977).

[18] D. van Dyck, Image Calculations in High-Resolution Electron Microscopy: Problems, Progress, and Prospects, in Advances in Electronics and Electron Physics, herausgegeben von P. W. Hawkes, Band 65, Seiten 295-355, Academic Press, Orlando, 1985.

[19] H. Löwen und T. Beier, Crystallinity in liquid films, Phys. Rev. B 41, 4435 (1990).

[20] F. Spaepen, Homogeneous nucleation and the temperature dependence of the crystal-melt interfacial tension, Solid State Phys. 47, 1 (1994). 
[21] J. M. Howe, Direct observation of order in the liquid at a solid-liquid interface by high-resolution transmission electron microscopy, Phil. Mag. A 74, 761 (1996).

[22] W. J. Huisman, J. F. Peters, M. J. Zwanenburg, S. A. de Vries, T. E. Derry, D. Abernathy und J. F. van der Veen, Layering of a liquid metal in contact with a hard wall, Nature 390, 379 (1997).

[23] P. Geysermans, D. Gorse und V. Pontikis, Molecular dynamics study of the solidliquid interface, J. Chem. Phys. 113, 6382 (2000).

[24] B. Plikat, Hochauflösende Transmissionselektronenmikroskopie an den kristallinamorphen Grenzflächen c-Si/a-Ge und c-Si/a-SiO${ }_{2}$, Dissertation, Universität Göttingen, 1998.

[25] B. Plikat, N. Borgardt, M. Seibt, W. Schröter und T. Wagner, The Modified Structure of Amorphous Ge near Si(111) Substrates, Inst. Phys. Conf. Ser. 164, 43 (1999).

[26] G. Etherington, A. Wright, J. Wenzel, J. Dore, J. Clarke und R. Sinclair, A neutron diffraction study of the structure of evaporated amorphous germanium, J. Non-Cryst. Solids 48, 265 (1982).

[27] B. Efron, Bootstrap Methods: Another Look at the Jacknife, Ann. Statist. 7, 1 (1979).

[28] B. Efron und R. Tibshirani, Bootstrap Methods for Standard Errors, Confidence Intervals, and Other Measures of Statistical Accuracy, Stat. Sci. 1, 54 (1986).

[29] W. Press, W. Vetterling, S. Teukolsky und B. Flannery, Numerical Recipes in C, Cambridge University Press, New York, 2. Edition, 1992.

[30] T. Proffen und S. J. L. Billinge, PDFFIT, a program for full profile structural refinement of the atomic pair distribution function, J. Appl. Cryst. 32, 572 (1999).

[31] V. Petkov, S. J. L. Billinge, S. D. Shastri und B. Himmel, High-resolution atomic distribution functions of disordered materials by high-energy X-ray diffraction, J. Non-Cryst. Solids 293-295, 726 (2001).

[32] V. Petkov, D. Qadir und S. D. Shastri, Rapid structure determination of disordered materials: study of GeSe $e_{2}$ glass, Solid State Commun. 129, 239 (2004).

[33] V. A. Levashov, S. J. L. Billinge und M. F. Thorpe, Density fluctuations and the pair distribution function, Phys. Rev. B 72, 024111 (2005). 
[34] A. Filipponi, F. Evangelisti, M. Benfatto, S. Mobilio und C. R. Natoli, Structural investigation of a-Si and a-Si:H using x-ray-absorption spectroscopy at the Si K edge, Phys. Rev. B 40, 9636 (1989).

[35] M. M. J. Treacy und J. M. Gibson, Variable Coherence Microscopy: a Rich Source of Structural Information from Disordered Materials, Acta Cryst. A52, 212 (1996).

[36] J. M. Gibson und M. M. Treacy, Diminished Medium-Range Order Observed in Annealed Amorphous Germanium, Phys. Rev. Lett. 78, 1074 (1997).

[37] M. M. J. Treacy, J. M. Gibson, L. Fan, D. J. Paterson und I. McNulty, Fluctuation microscopy: a probe of medium range order, Rep. Prog. Phys. 68, 2899 (2005).

[38] Y. Tu, J. Tersoff, G. Grinstein und D. Vanderbilt, Properties of a ContinuousRandom-Network Model for Amorphous Systems, Phys. Rev. Lett. 81, 4899 (1998).

[39] W. H. Zachariasen, The atomic arrangement in glass, J. Am. Chem. Soc. 54, 3841 (1932).

[40] N. Bernstein, M. J. Aziz und E. Kaxiras, Amorphous-crystal interface in silicon: A tight-binding simulation, Phys. Rev. B 58, 4579 (1998).

[41] C. R. da Silva und A. Fazzio, Formation and structural properties of the amorphous-crystal interface in a nanocrystalline system, Phys. Rev. B 64, 075301 (2001).

[42] S. Izumi, S. Hara, T. Kumagai und S. Sakai, Structural and mechanical properties of well-relaxed amorphous-crystal interface in silicon: molecular dynamics study, Comp. Mater. Sci. 31, 279 (2004).

[43] S. Kret, P. Ruterana, A. Rosenauer und D. Gerthsen, Extracting Quantitative Information from High Resolution Electron Microscopy, phys. stat. sol. (b) 227, 247 (2001).

[44] K. Scheerschmidt, Retrieval of object information by inverse problems in electron diffraction, J. Microsc. 190, Pts 1/2, 238 (1998).

[45] O. Scherzer, The Theoretical Resolution Limit of the Electron Microscope, J. Appl. Phys. 20, 20 (1949).

[46] H. Lichte, Optimum focus for taking electron holograms, Ultramicroscopy 38, 13 (1991).

[47] H. Alexander, Physikalische Grundlagen der Elektronenmikroskopie, Teubner Studienbücher, Stuttgart, 1. Edition, 1997. 
[48] P. Schwander, C. Kisielowski, M. Seibt, F. Baumann, Y. Kim und A. Ourmazd, Mapping projected potential interfacial roughness and composition in general crystalline solids by quantitative transmission electron microscopy, Phys. Rev. Lett. 71, 4150 (1993).

[49] C. Kisielowski, P. Schwander, F. Baumann, M. Seibt, Y. Kim und A. Ourmazd, An approach to quantitative high-resolution transmission electron microscopy of crystalline materials, Ultramicroscopy 58, 131 (1995).

[50] D. Stenkamp und H. P. Strunk, Quantitative determination of defocus, thickness and composition from high-resolution transmission electron microscopy lattice images, Appl. Phys. A 62, 369 (1996).

[51] K. Thiel, Quantitative Analyse elektronenmikroskopischer Abbildungen von Grenzflächen zwischen amorphen und kristallinen Festkörpern, Diplomarbeit, Universität Göttingen, 2000.

[52] A. Ourmazd, D. W. Taylor und J. Cunningham, Chemical Mapping of Semiconductor Interfaces at Near-Atomic Resolution, Phys. Rev. Lett. 62, 933 (1989).

[53] A. Ourmazd, F. Baumann, M. Bode und Y. Kim, Quantitative chemical lattice imaging: theory and practice, Ultramicroscopy 34, 237 (1990).

[54] A. Rosenauer, T. Remmele, D. Gerthsen, K. Tillmann und A. Förster, Atomic scale strain measurements by the digital analysis of transmission electron microscopic lattice images, Optik 105, 99 (1997).

[55] H. Seitz, Chemische- und Verzerrungsanalyse mit nahezu atomarer Auflösung in der hochauflösenden Elektronenmikroskopie, Diplomarbeit, Universität Göttingen, 1992.

[56] R. Bierwolf, M. Hohenstein, F. Phillipp, O. Brandt, G. E. Crook und K. Ploog, Direct measurement of local lattice distortions in strained layer structures by HREM, Ultramicroscopy 49, 273 (1993).

[57] P. Bayle, T. Deutsch, B. Gilles, F. Lançon, A. Marty und J. Thibault, Quantitative analysis of the deformation and chemical profiles of strained multilayers, Ultramicroscopy 56, 94 (1994).

[58] P. H. Jouneau, A. Tardot, G. Feuillet, H. Mariette und J. Cibert, Strain Mapping of Ultrathin Epitaxial ZnTe and MnTe Layers Embedded in CdTe, J. Appl. Phys. 75, 7310 (1994). 
[59] H. Seitz, M. Seibt, F. Baumann, K. Ahlborn und W. Schröter, Quantitative strain mapping using high-resolution electron-microscopy, phys. stat. sol. (a) 150, 625 (1995).

[60] A. Rosenauer, S. Kaiser, T. Reisinger, J. Zweck, W. Gebhardt und D. Gerthsen, Digital analysis of high resolution transmission electron microscopy lattice images, Optik 102, 63 (1996).

[61] H. Seitz, K. Ahlborn, M. Seibt und W. Schröter, Sensivity limits of strain mapping procedures using high-resolution electron microscopy, J. Microsc. 190, Pts 1/2, 184 (1998).

[62] H. Lichte, Electron holography : I. Can electron holography reach $0.1 \mathrm{~nm}$ resolution?, Ultramicroscopy 47, 223 (1992).

[63] H. Lichte, E. Völkl und K. Scheerschmidt, Electron holography : II. First steps of high resolution electron holography into materials science, Ultramicroscopy $\mathbf{4 7}$, 231 (1992).

[64] P. Schiske, Zur Frage der Bildrekonstruktion durch Fokusreihen, in Proc. $4^{\text {th }}$ Eur. Conf. On Electron Microscopy, Rome, Seite 145, 1968.

[65] P. Schiske, in Image Processing and Computer-Aided Design in Electron Optics, herausgegeben von P. W. Hawkes, Seite 82, Academic Press, London, 1973.

[66] W. O. Saxton, Computer Techniques for Image Processing in Electron Microscopy, Kapitel 9.7, Academic Press, New York, 1978.

[67] W. O. Saxton, in Proc. 11th Int. Congr. on Electron Microscopy, Post-Deadline Paper 1-4, Kyoto, Japan, 1986.

[68] W. O. Saxton, Linear image restoration: the approaches available for real and complex objects, in Image Interpretation and Image Processing in Electron Microscopy, Autum School 1992, herausgegeben von J. Heydenreich und W. Neumann, Seite 118, Max Planck Institut für Mikrostrukturphysik, Halle/Saale, 1993.

[69] E. J. Kirkland, Improved high resolution image processing of bright field electron micrographs : I. Theory, Ultramicroscopy 15, 151 (1984).

[70] E. J. Kirkland, B. M. Siegel, N. Uyeda und Y. Fujiyoshi, Improved high resolution image processing of bright field electron micrographs : II. Experiment, Ultramicroscopy 17, 87 (1985). 
[71] E. J. Kirkland, Scanning Microscopy Suppl. 2 (1988), in Proc. Sixth Pfefferkorn Conference on Image and Signal Processing in Electron Microscopy, Seite 139, Niagra Falls, Canada, 1987.

[72] M. Lentzen und K. Urban, Reconstruction of the projected crystal potential in transmission electron microscopy by means of a maximum-likelihood refinement algorithm, Acta Cryst. A56, 235 (2000).

[73] A. Thust, M. H. F. Overwijk, W. M. J. Coene und M. Lentzen, Numerical correction of lens aberrations in phase-retrieval HRTEM, Ultramicroscopy 64, 249 (1996).

[74] R. Rosenfeld, A.Thust, W. Yang, M. Feuerbacher und K. Urban, Investigation of the cluster substructure of icosahedral Al-Pd-Mn quasicrystals by means of exitplane wavefunction reconstruction in high-resolution electron microscopy, Phil. Mag. Lett. 78, 127 (1998).

[75] C. L. Jia, R. Rosenfeld, A. Thust und K. Urban, Atomic structure of a $\Sigma=3,\{111\}$ twin-boundary junction in a BaTiO 3 thin film, Phil. Mag. Lett. 79, 99 (1999).

[76] C. L. Jia und A. Thust, Investigation of Atomic Displacements at a $\Sigma 3\{111\}$ Twin Boundary in $\mathrm{BaTiO}_{3}$ by Means of Phase-Retrieval Electron Microscopy, Phys. Rev. Lett. 82, 5052 (1999).

[77] P. Stadelmann, EMS - a software package for electron diffraction analysis and HREM image simulation in materials science, Ultramicroscopy 21, 131 (1987).

[78] J. Frank, The Role of Correlation Techniques in Computer Image Processing, in Computer Processing of Electron Microscope Images (Topics in Current Physics 13), herausgegeben von P. Hawkes, Kapitel 5, Seiten 187-222, Springer-Verlag, 1980.

[79] R. Meyer, A. Kirkland und W. Saxton, A new method for the determination of the wave aberration function for high-resolution TEM. 1. Measurement of the symmetric aberrations, Ultramicroscopy 92, 89 (2002).

[80] M. J. Hÿtch und W. M. Stobbs, Quantitative comparison of high-resolution TEM images with simulations, Ultramicroscopy 53, 191 (1994).

[81] M. J. Hÿtch und W. M. Stobbs, Quantitative criteria for the matching of simulations with experimental HREM images, Microsc. Microanal. Microstruct. 5, 133 (1994). 
[82] G. Möbus und M. Rühle, Structure determination of metal-ceramic interfaces by numerical contrast evaluation of HRTEM micrographs, Ultramicroscopy 56, 54 (1994).

[83] R. Hillebrand und H. Hofmeister, Quantitative Analysis of HREM Images: Measures of Similarity, phys. stat. sol. (a) 150, 65 (1995).

[84] D. Hofmann und F. Ernst, Quantitative high-resolution transmission electron microscopy of the incoherent $\Sigma 3$ (211) boundary in $\mathrm{Cu}$, Ultramicroscopy 53, 205 (1994).

[85] G. Möbus und G. Dehm, Retrieval of crystal defect structures from HREM images by simulated evolution II. Experimental image evaluation, Ultramicroscopy 65, 217 (1996).

[86] W. E. King, G. H. Campbell, S. M. Foiles, D. Cohen und K. M. Hanson, Quantitative HREM observation of the $\sigma 11(113) /[1 \overline{1} 0]$ grain-boundary structure in aluminium and comparison with atomistic simulation, J. Microsc. 190, Pts 1/2, 131 (1998).

[87] G. Möbus und O. Kienzle, Interface structure retrieval by HREM: From entropy maximisation to r-factor fits, in Proceedings of EMAG 1999, herausgegeben von C. J. Kiely, Seiten 263-266, Sheffield, 1999, IOP, Bristol, UK.

[88] G. Möbus, Probability calculus for quantitative HREM.Part II: Entropy and likelihood concepts, Ultramicroscopy 85, 199 (2000).

[89] C. Boothroyd, R. Dunin-Borkowski, W. Stobbs und C. Humphreys, Quantifying the effects of amorphous layers on image contrast using energy filtered transmission electron microscopy, Band 354 der MRS Symposium Proceedings, Seiten 495-500, Materials Research Society, Pittsburgh, 1995.

[90] K. von Hochmeister und F. Phillip, Quantitative comparison of HREM images with image simulations, in Proc. XI European Congress on Microscopy, Dublin, 26-30 August 1996, EUREM 96, UCD, Dublin, 1996.

[91] C. Boothroyd, Why don't high-resolution simulations and images match?, J. Microsc. 190, Pts 1/2, 99 (1998).

[92] R. R. Meyer, J. Sloan, R. E. Dunin-Borkowski, A. I. Kirkland, M. C. Novotny, S. R. Bailey, J. L. Hutchison und M. L. H. Green, Discrete Atom Imaging of OneDimensional Crystals Formed Within Single-Walled Carbon Nanotubes, Science 289, 1324 (2000). 
[93] C. B. Boothroyd, Quantification of high-resolution electron microscope images of amorphous carbon, Ultramicroscopy 83, 159 (2000).

[94] M. Lehmann, D. Geiger, I. Büscher, H. Zandbergen, D. V. Dyck und H. Lichte, Quantitative Analysis of Focal-Series of Off-axis Electron Holograms, in Proc. $15^{\text {th }}$ Int. Congr. on Electron Microscopy ICEM15, Band 3, Seiten 279-280, Durban, South Africa, 2002.

[95] C. B. Boothroyd, Quantification of lattice images: the contribution from diffuse scattering, J. Electron Microsc. 51, S279 (2002).

[96] A. Howie, Hunting the Stobbs factor, Ultramicroscopy 98, 73 (2004).

[97] A. Harscher, F. Lenz und H. Lichte, Electron Holography provides Zero-LossImages, in Proceedings of the $10^{\text {th }}$ European Conference on Electron Microscopy EUREM'92, Granada, Spanien, 1992.

[98] Z. L. Wang, Thermal diffuse scattering in high-resolution electron holography, Ultramicroscopy 52, 504 (1993).

[99] A. Ourmazd, D. W. Taylor, J. A. Rentschler und J. Bevk, $\mathrm{Si} \rightarrow \mathrm{SiO}_{2}$ transformation: Interfacial structure and mechanism, Phys. Rev. Lett. 59, 213 (1987).

[100] N. Ikarashi, K. Watanabe und Y. Miyamoto, High-resolution transmission electron microscopy of an atomic structure at a Si(001) oxidation front, Phys. Rev. B 62, 15989 (2000).

[101] H. Akatsu, Y. Sumi und I. Ohdomari, Evaluation of SiO $2 /(001)$ Si interface roughness using high-resolution transmission electron microscopy and simulation, Phys. Rev. B 44, 1616 (1991).

[102] N. Nakanishi, Y. Kikuchi, T. Yamazaki, E. Okunishi, K. Watanabe und I. Hashimoto, Quantitative evaluation of $\mathrm{SiO}_{2} / \mathrm{Si}$ interfaces using high-resolution high-angle annular dark field scanning transmission electron microscopy, Phys. Rev. B 70, 165324 (2004).

[103] I. Ohdomari, H. Akatsu, Y. Yamakoshi und K. Kishimoto, Study of the interfacial structure between $\mathrm{Si}(100)$ and thermally grown $\mathrm{SiO}_{2}$ using a ball-and-spoke model, J. Appl. Phys. 62, 3751 (1987).

[104] W. Hoppe, R. Langer, J. Frank und A. Feltynowski, Bilddifferenzverfahren in der Elektronenmikroskopie, Naturwiss. 56, 267 (1969). 
[105] L. S. Al-Ali, Translational alignment of differently defocused micrographs using cross-correlation, in Developments in Electron Microscopy and Analysis, herausgegeben von J. A. Venables, Seiten 225-228, Proceedings of EMAG 75, Academic Press, 1976.

[106] K. Thiel, N. Borgardt, B. Plikat und M. Seibt, Quantitative characterisation of a crystalline-amorphous interface by high-resolution electron microscopy, in Vorbereitung (2006).

[107] C. R. Hubbard, H. E. Swanson und F. A. Mauer, A silicon powder diffraction standard reference material, J. Appl. Cryst. 8, 45 (1975).

[108] E. J. Kirkland, Advanced Computing in Electron Microscopy, Plenum Press, New York, 1998.

[109] K. Laaziri, S. Kycia, S. Roorda, M. Chicoine, J. L. Robertson, J. Wang und S. C. Moss, High-energy x-ray diffraction study of pure amorphous silicon, Phys. Rev. B 60, 13520 (1999).

[110] J. C. Dainty und R. Shaw, Image Science, Seite 211ff, Academic Press, New York, 1974.

[111] A. L. Weickenmeier, W. Nüchter und J. Mayer, Quantitative characterization of point spread function and detection quantum efficiency for a YAG scintillator slow scan CCD camera, Optik 99, No. 4, 147 (1995).

[112] J. M. Zuo, Electron detection characteristics of slow-scan CCD camera, Ultramicroscopy 66, 21 (1996).

[113] M. Seibt, Messung der Modulationstransferfunktion der SSC CCD, private Mitteilung, 1999.

[114] O. L. Krivanek und P. E. Mooney, Applications of slow scan CCD cameras in transmission electron microscopy, Ultramicroscopy 49, 95 (1993).

[115] V. F. Sears und S. A. Shelley, Debye-Waller Factor for Elemental Crystals, Acta Cryst. A47, 441 (1991).

[116] J. A. Nelder und R. Mead, A simplex method for function minimization, Computer Journal 7, 308 (1965).

[117] J. R. Taylor, An introduction to error analysis, University Science Books, Sausalito, California, 1982. 
[118] M. Suhren, D. Gräf, R. Schmolke, H. Piontek und P. Wagner, Applications of Atomic Force Microscopy for Silicon Wafer Characterization, Inst. Phys. Conf. Ser. 149, 301 (1996).

[119] W. Schweika, Schichtpräparation mit Sputterverfahren, in Magnetische Schichtsysteme, 30. Ferienkurs des Instituts für Festkörperforschung, Forschungszentrum Jülich, 1999.

[120] M. Lentzen, B. Jahnen, C. L. Jia, A. Thust, K. Tillmann und K. Urban, Highresolution imaging with an aberration-corrected transmission electron microscope, Ultramicroscopy 92, 233 (2002).

[121] M. Lentzen, Progress in Aberration-Corrected High-Resolution Transmission Electron Microscopy Using Hardware Aberration Correction, Microsc. Microanal. 12, 191 (2006).

[122] J. Spence, Experimental High-Resolution Electron Microscopy, Oxford University Press, New York, 2. Edition, 1988.

[123] O. Krivanek, A method for determining the coefficient of spherical aberration from a single electron micrograph, Optik 45, 97 (1976).

[124] W. Coene und T. Denteneer, Improved methods for the determination of the spherical aberration coefficient in high-resolution electron microscopy from micrographs of an amorphous object, Ultramicroscopy 38, 225 (1991).

[125] J. M. Gibson, Breakdown of the weak-phase object approximation in amorphous objects and measurement of high-resolution electron optical parameters, Ultramicroscopy 56, 26 (1994).

[126] L. Korte, Private Mitteilung, 2006.

[127] M. Seibt, Elektronenmikroskopische Untersuchungen des Ausscheidungsverhaltens von Nickel in Silizium, Dissertation, Universität Göttingen, 1986.

[128] U. Gnauert, Metastabile Phasenbildung und metallinduzierte Kristallisation in Silizium-Gold-Vielfachschichten, Dissertation, Universität Göttingen, 1994.

[129] J. Glander, Aufbau einer Molekularstrahlepitaxie-Anlage und Untersuchung der Phasenbildung während des Wachsens von kodeponierten Au/Si-Schichten auf kristallinem Silizium, Dissertation, Universität Göttingen, 1998. 


\section{Danksagungen}

Eine Arbeit wie die hier vorliegende kann nur aus der Kombination von kreativer Forschung und einer produktiven Zusammenarbeit verschiedener Personen entstehen. Im Folgenden möchte ich die Gelegenheit nutzen, mich bei diesen Menschen zu bedanken - ohne den Anspruch auf Vollständigkeit zu erheben...

- Mein erster Dank geht selbstverständlich an PD Dr. Michael Seibt, meinen Doktorvater, der die Untersuchungen ermöglicht und durch sein kontinuierliches Interesse am Fortgang dieser Arbeit sowie seine stete Diskussionsbereitschaft grundlegend zu ihrem Gelingen beigetragen hat.

- Herrn Prof. Dr. Tim Salditt danke ich für die Übernahme des Korreferats.

- Prof. Dr. Nikolai Borgardt, dem „Vater“ der APP-Näherung, möchte ich für die sehr fruchtbare Zusammenarbeit in den letzten Jahren danken. Die wenigen Wochen pro Jahr, in denen er sich in Göttingen aufhielt, waren zwar immer sehr anstrengend, aber dafür äußerst produktiv. Ohne seine Ideen wäre vieles nicht entstanden.

- Für viele interessante Diskussionen und Gespräche - nicht nur physikalischen Inhalts sei Prof. Dr. Wolfgang Schröter gedankt.

- An Dipl.-Phys. Tore Niermann, meinen langjährigen Zimmergenossen, geht ein Dank für die angenehme Arbeitsatmosphäre und die sehr gute Zusammenarbeit bei diversen gemeinsamen Projekten.

- Der gesamten Arbeitsgruppe Seibt, aktuell bestehend aus den Herren Dipl.-Phys. Tore Niermann, Dipl.-Phys. Carsten Rudolf, Dipl.-Phys. Oliver Voß, Dipl.-Phys. cand. Henning Schuhmann, Dipl.-Phys. cand. Philipp Saring und Dipl.-Phys. cand. Hendrik Bergmann, danke ich vielmals für die kritische Durchsicht des Manuskripts sowie viele interessante Diskussionen physikalischer und außerphysikalischer Art.

- Den nicht namentlich genannten restlichen Mitgliedern des IV. Physikalischen Instituts danke ich für die gute Zusammenarbeit.

- Für die Herstellung der a-Si/c-Si-Proben geht mein Dank an Dr. Lars Korte.

- Ein besonderer Dank geht an meine Schwester StudR' Stephanie Goldmann und an meinen Schwager Dr. des. Frank Goldmann für die sprachliche Korrektur des Manuskripts.

Zum Schluss danke ich meinen Eltern und meiner Schwester, nicht nur für die finanzielle Ermöglichung meiner Ausbildung, sondern auch für die fortwährende Unterstützung während der Promotionszeit.

Der größte Dank geht aber an meine Freundin Sandra, die mich in den letzten Jahren immer wieder unterstützt und aufgebaut hat, wenn ich dachte, es geht nicht mehr weiter. Ich hoffe, ich kann mich irgendwann revanchieren... 



\section{Lebenslauf}

\section{PERSÖNLICHE ANGABEN}

Name: Karsten Thiel

Familienstand: ledig

Staatsangehörigkeit: deutsch

Geburtsdatum: 20.07.1971

Geburtsort: Bremen

$\underline{\text { SCHULAUSBILDUNG }}$

$\begin{array}{ll}1977-1981 & \text { Grundschule Auf der Hohwisch, Bremen } \\ 1981-1983 & \text { Orientierungsstufe Alter Postweg, Bremen } \\ 1983-1990 & \text { Gymnasium Altes Gymnasium, Bremen }\end{array}$

WEHRDIENST

$1990-1991 \quad$ Grundwehrdienst in Schwanewede

\section{HOCHSCHULAUSBILDUNG}

Studium der Physik an der Georg-August-Universität zu Göttingen

WS 1991/1992

Aufnahme des Studiums

$07 / 1994$

Vordiplom

$02 / 2000$

Diplom, Thema der Diplomarbeit:

"Quantitative AnALYse ELEKTRONENMIKROSKOPISCHER ABBILDUNGEN VON

GRENZFLÄCHEN ZWISCHEN AMORPHEN UND KRISTALLINEN FESTKÖRPERN"

02/2000 - 06/2001 Wissenschaftliche Hilfskraft am IV. Physikalischen Institut

$10 / 2000$ Beginn der vorliegenden Dissertation

seit $07 / 2001$ Wissenschaftlicher Angestellter der Universität Göttingen 
seit $01 / 2002$

$01 / 2002-12 / 2004$

seit $01 / 2005$
Mitarbeiter im Sonderforschungsbereich 602: „KOMPleXe StrukTUREN IN KONDENSIERTER MATERIE VON ATOMARER BIS MESOSKOPISCHER SKALA"

im Teilprojekt A13:

„STruktur VON Grenzflächen ZWISCHEN GEORDNETEN UND UNGEORDNETEN FESTKÖRPERN"

im Teilprojekt A14:

„Struktur und Transport in TunnelMAGNETOWIDERSTANDSELEMENTEN MIT ULTRADÜNNEN BARRIEREN" 